\title{
Optimal contract design in the joint economic lot size problem with multi-dimensional asymmetric information
}

DOI:

10.1016/j.ejor.2016.02.053

Document Version

Accepted author manuscript

Link to publication record in Manchester Research Explorer

\section{Citation for published version (APA):}

Pishchulov, G., \& Richter, K. (2016). Optimal contract design in the joint economic lot size problem with multidimensional asymmetric information. European Journal of Operational Research, 253(3), 711-733.

https://doi.org/10.1016/j.ejor.2016.02.053

\section{Published in:}

European Journal of Operational Research

\section{Citing this paper}

Please note that where the full-text provided on Manchester Research Explorer is the Author Accepted Manuscript or Proof version this may differ from the final Published version. If citing, it is advised that you check and use the publisher's definitive version.

\section{General rights}

Copyright and moral rights for the publications made accessible in the Research Explorer are retained by the authors and/or other copyright owners and it is a condition of accessing publications that users recognise and abide by the legal requirements associated with these rights.

\section{Takedown policy}

If you believe that this document breaches copyright please refer to the University of Manchester's Takedown Procedures [http://man.ac.uk/04Y6Bo] or contact uml.scholarlycommunications@manchester.ac.uk providing relevant details, so we can investigate your claim.

\section{OPEN ACCESS}




\title{
Optimal contract design in the joint economic lot size problem with multi-dimensional asymmetric information
}

\author{
Grigory Pishchulov ${ }^{\mathrm{a}, *}$, Knut Richter ${ }^{\mathrm{b}}$ \\ ${ }^{a}$ TU Dortmund University, Faculty of Business, Economics and Social Sciences, D-44227 Dortmund, Germany \\ ${ }^{b}$ St. Petersburg State University, 7/9 Universitetskaya nab., St. Petersburg, 199034 Russia
}

\begin{abstract}
Previous work has studied the classical joint economic lot size model as an adverse selection problem with asymmetric cost information. Solving this problem is challenging due to the presence of countervailing incentives and two-dimensional information asymmetry, under which the classical single-crossing condition does not need to hold. In the present work we advance the existing knowledge about the problem on hand by conducting its optimality analysis, which leads to a better informed and an easier problem solution: First, we refine the existing closed-form solution, which simplifies problem solving and its analysis. Second, we prove that Karush-Kuhn-Tucker conditions are necessary for optimality, and demonstrate that the problem may, in general, possess non-optimal stationary points due to non-convexity. Third, we prove that certain types of stationary points are always dominated, which eases the analytical solution of the problem. Fourth, we derive a simple optimality condition stating that a weak Pareto efficiency of the buyer's possible cost structures implies optimality of any stationary point. It simplifies the analytical solution approach and ensures a successful solution of the problem by means of conventional numerical techniques, e.g. with a general-purpose solver. We further establish properties of optimal solutions and indicate how these are related with the classical results on adverse selection.
\end{abstract}

Keywords: supply chain coordination, asymmetric information, nonlinear programming

\section{Introduction}

Banerjee's joint economic lot size model (Banerjee, 1986) refers to a bilateral monopoly consisting of a single buyer operating in an EOQ setting and a single supplier who manufactures and ships to order. If the buyer's economic order quantity (EOQ) and the supplier's economic lot size (ELS) do not coincide then the parties should negotiate the lot size to be adopted for repetitive ordering, manufacturing and shipment of the product within this supply chain. As long as the

\footnotetext{
${ }^{*}$ Corresponding author. Tel.: +49 231755 3234; fax: +49 2317553189.

Email addresses: grigory.pishchulov@tu-dortmund.de (Grigory Pishchulov), k.rihter@spbu.ru (Knut Richter)
} 
buyer (referred to as he) has decision authority with regard to the order size, he will insist on his EOQ. The supplier (she) can still do better by offering the buyer a side payment as a reward for deviating from his EOQ to the system-optimal joint economic lot size (JELS), and thus realize a Pareto improvement. The JELS model has received a considerable attention in the literature as a conventional model of supply chain coordination and has further been extended to a number of more general settings (Ben-Daya et al., 2008; Glock, 2012; Liu and Çetinkaya, 2007; Pibernik et al., 2011; Sucky, 2004, 2006).

In particular, Sucky $(2004,2006)$ studied a setting in which the buyer is privately informed of his cost structure and can therefore abuse this information asymmetry by untruthful reporting of cost data, and thus induce favorable terms of contract with the supplier. To counteract, the supplier can proactively design a menu of offers, each comprising a particular lot size and a side payment, for presenting to the buyer. Determining such a menu of offers that would minimize the supplier's own expected costs constitutes her bargaining problem. It has been modeled and solved in Sucky $(2004,2006)$ as a principal-agent problem in adverse selection form (Laffont and Martimort, 2002).

Sucky's work thus studies a conventional supply chain coordination model by Banerjee in a more general and a more realistic setting, and parallels much of the supply chain research that addresses information asymmetry in various settings (see e.g. Burnetas et al., 2007; Cachon and Zhang, 2006; Corbett and de Groote, 2000; Ha, 2001; Lau et al., 2006; Liu and Cetinkaya, 2009; Lutze and Özer, 2008; Voigt and Inderfurth, 2011; Wang et al., 2009; Yang et al., 2009). At the same time, it has a distinctive feature of assuming information asymmetry to be two-dimensional, i.e. with respect to holding and fixed ordering costs of the buyer. As a result, the bargaining problem in Sucky (2004, 2006) gives rise to a non-linear optimization problem whose solution poses certain challenges. In particular, as the problem proves to be non-convex, its stationary points do not need to be optimal. Consequently, the analytical solution as in Sucky $(2004,2006)$ requires, in general, to determine and evaluate all stationary points of the problem, whereas attempting to solve it numerically may result in a convergence to a non-optimal solution.

These particular model properties have not, however, received attention in the follow-up research. Due to the significance of the JELS model, we believe that they deserve a closer investigation. Therefore, the present work intends to advance our knowledge of the bargaining problem under consideration and offer a better informed and an easier problem solution. Specifically, in Sucky $(2004,2006)$, a detailed analysis has been conducted for the setting in which two possible cost structures are assumed for the buyer. In the present work we (i) propose a refinement of the original closed-form solution, which simplifies problem solving and its analysis; (ii) demonstrate that the problem on hand may possess non-optimal stationary points due to non-convexity; 
(iii) prove that certain types of stationary points are always dominated; (iv) establish an easily perceivable sufficient optimality condition, under which any stationary point of the problem proves to be its optimal solution - despite of non-convexity of the problem. Result (iii), in particular, eases the analytical solution of the problem by allowing to restrict attention only to specific types of stationary points. Result (iv), in turn, ensures a successful solution of the problem by means of conventional numerical techniques, e.g. with a general-purpose solver, when the said condition holds true, and also simplifies the analytical solution approach. We further establish properties of optimal solutions and indicate how these are related with the classical results on adverse selection.

The rest of the paper is organised as follows. Section 2 provides an overview of adverse selection problems. In Section 3 we introduce the problem setting, describe the solution approach as per Sucky $(2004,2006)$ and present a refined closed-form solution. In Section 4 we discuss the nonconvexity of the problem on hand, identify solution properties and relate them with the classical results on adverse selection. In Section 5 we establish a sufficient condition for optimality of a stationary point. Section 6 provides concluding remarks.

\section{Adverse selection problems}

The principal-agent problem in adverse selection form has received an extensive treatment in the information economics literature and found a broad range of applications in diverse areas (Rochet and Stole, 2003; van Ackere, 1993). In its typical setup (Laffont and Martimort, 2002), a principal sets out to contract an agent to produce some quantity of a certain good. The parties need to settle on the contract terms $(x, z)$, where $x$ is the quantity to be produced and $z$ is the payment to the agent. The principal's valuation of the quantity $x$ is $S(x)$. The agent's production costs are $C(\theta, x)$, where $\theta \in \Theta$ is the agent type that is privately known by the agent; the principal has only knowledge of the probability distribution $\Omega$ over $\Theta$.

The principal has all of the bargaining power. The principal's problem is to design and announce a mechanism $(M, g(m))$ with a message space $M$ and allocation rule $g: m \mapsto(x, z)$ that establishes the contract terms depending on the message $m \in M$ to be received from the agent. The objective of mechanism design is maximization of the principal's own expected utility $\mathbb{E}_{\theta}[S(x)-z]$. By the revelation principle (Myerson, 1979, 1982), the principal can restrict attention to truthful direct revelation mechanisms, i.e. mechanisms with $M=\Theta$ such that the agent weakly prefers revealing his true type to revealing any other type in the message $m$.

Given a finite set $\Theta=\left\{\theta_{1}, \ldots, \theta_{n}\right\}$ of possible agent types with respective probabilities $\omega_{1}, \ldots, \omega_{n}$, the problem can be stated as one of designing an incentive-feasible menu of contracts $\left\{\left(x_{i}, z_{i}\right)\right\}_{i=1}^{n}$ for presenting to the agent, where contracts are linked to agent types in a way that the agent 
self-selects by choosing the contract designed for his true type. Specifically, the principal solves:

$$
\begin{array}{rll}
\max _{\left\{\left(x_{i}, z_{i}\right)\right\}} & \sum_{i=1}^{n}\left[S\left(x_{i}\right)-z_{i}\right] \cdot \omega_{i} & \\
\text { s.t. } & z_{i}-C\left(\theta_{i}, x_{i}\right) \geq \underline{u}_{i} & \forall i \\
& z_{i}-C\left(\theta_{i}, x_{i}\right) \geq z_{j}-C\left(\theta_{i}, x_{j}\right) & \forall i, j \quad(i \neq j)
\end{array}
$$

In the above problem, objective $(\mathbb{O})$ is to maximize the principal's expected utility from the contract selected by the agent. Constraints $\left(\mathrm{IR}_{i}\right)$ respect the agent's individual rationality by ensuring that the agent of any type $i$ gets at least his reservation utility $\underline{u}_{i}$ when accepting the $i$-th contract. Constraints $\left(\mathrm{IC}_{i j}\right)$ further ensure that the menu of contracts is incentive-compatible, so that the agent of any type $i$ weakly prefers the $i$-th contract to any other contract from the menu.

Problem $(\mathbb{O}),\left(\mathrm{IR}_{i}\right)-\left(\mathrm{IC}_{i j}\right)$ can be solved particularly easy when the following additional assumptions hold:

i) $C(\theta, x)$ is convex in $x$, for each $\theta \in \Theta$, and $S(x)$ is concave.

ii) Reservation utility is the same for all agent types: $\underline{u}_{1}=\ldots=\underline{u}_{n}$.

iii) Information asymmetry is one-dimensional, so that agent type is represented by a scalar $\theta$.

iv) The single-crossing condition is satisfied: $C_{x}(\theta, x)$ is strictly increasing in $\theta$, for each $x .^{1}$

v) $C(\theta, x)$ is strictly increasing in $\theta$ and $C_{x x}(\theta, x)$ is non-decreasing in $\theta$, for each $x$.

Note that assumption ii) allows to normalize all reservation utilities to zero, whereas assumption iii) implies, without loss of generality, a strict order $\theta_{1}<\ldots<\theta_{n}$ of the agent types. Assumption iv) states that indifference curves of any two agent types on the $(x, z)$-plane cross at most once. ${ }^{2}$

With $n=2$, it can then be shown that constraints $\left(\mathrm{IR}_{2}\right)$ and $\left(\mathrm{IC}_{12}\right)$ bind at the optimum, while $\left(\mathrm{IR}_{1}\right)$ and $\left(\mathrm{IC}_{21}\right)$ do not, by virtue of assumptions iv), v) and monotonicity of the principal's utility in $z$. Eliminating variables $z_{1}, z_{2}$ via binding constraints gives a concave function in $(\mathbb{O})$ due to assumptions i) and v); its unconstrained maximization yields optimal quantities $\hat{x}_{1}, \hat{x}_{2}$ to be offered to the respective agent types via a menu of contracts. These quantities are referred to as second-best, in contrast to the first-best quantities $x_{1}^{*}, x_{2}^{*}$ that would have been allocated under complete information. By virtue of assumption iv), it holds that $\hat{x}_{2}<x_{2}^{*}<x_{1}^{*}=\hat{x}_{1}$; thus, the agent types are separated via different contracts in the optimal menu, while the quantity contracted for with the less efficient type $\theta_{2}$ is distorted downwards from the first-best.

\footnotetext{
${ }^{1} C_{x}$ and $C_{x x}$ respectively designate the $1^{\text {st }}$ and $2^{\text {nd }}$ order partial derivatives of $C$ with respect to $x$.

${ }^{2}$ See in this regard e.g. Laffont and Martimort (2002) as well as Kreps (1990, Chapter 17). More generally, the single-crossing condition requires $C_{x}(\cdot, x)$ to be either strictly increasing for each $x$ or strictly decreasing for each $x$.
} 
These insights carry over to the case of $n>2$ as follows (Laffont and Martimort, 2002; Maskin and Riley, 1984): the individual rationality constraint $\left(\mathrm{IR}_{n}\right)$ of the highest agent type and the local upward incentive compatibility constraints $\left(\mathrm{IC}_{i, i+1}\right)$ for $i=1, \ldots, n-1$ must bind at the optimum. As a result, second-best allocation quantities to all agent types except for $\theta_{1}$ are distorted downwards from the first-best. The quantities are weakly monotonic in the agent type: $\hat{x}_{n} \leq \ldots \leq \hat{x}_{2}<\hat{x}_{1}$, where any equality leads to bunching of the respective types in terms of an identical contract intended for them in the optimal menu. The allocation quantities are, however, strictly monotonic if $C_{x}\left(\theta_{i}, x\right)+\frac{\Omega_{i-1}}{\omega_{i}}\left(C_{x}\left(\theta_{i}, x\right)-C_{x}\left(\theta_{i-1}, x\right)\right)$ is strictly increasing in $i$ for each $x$, where $\Omega_{i}=\sum_{j \leq i} \omega_{j}$. Depending on the setting, this can be ensured by the monotonicity of the hazard rate of probability distribution $\Omega$, i.e. by $\frac{\Omega_{i-1}}{\omega_{i}}$ being strictly increasing in $i$ (see e.g. Laffont and Martimort, 2002).

When $\Omega$ is a continuous probability distribution, it is being typically assumed that $\Theta=[\underline{\theta}, \bar{\theta}]$ (see Hellwig, 2010, for a mixed distribution of agent types). The problem is accordingly to determine an incentive-feasible schedule $(x(\theta), z(\theta))$ that maximizes the principal's expected utility. Problem $(\mathbb{O})$, $\left(\mathrm{IR}_{i}\right)-\left(\mathrm{IC}_{i j}\right)$ is then straightforward to adjust. Several solution approaches, featuring variational and control-theoretic methods, exist (see Laffont and Martimort, 2002; Rochet and Stole, 2003). The analysis of the problem and its solution leads under assumptions i) -v) to similar insights as in the discrete case indicated above.

Problem solution becomes, however, more involved when one or more of the assumptions i)-v) are violated. In the discrete case, this may require to use direct nonlinear programming methods (Bazaraa et al., 2006) or to resort to advanced numerical techniques (see e.g. Cecchini et al., 2013). In the continuous case, the analysis may turn out to be intractable (Laffont and Martimort, 2002). Research work has particularly addressed study settings in which one of assumptions ii)-iv) does not hold. Regarding ii), Kerschbamer and Maderner (1998) and Jullien (2000) studied the problem in the discrete and continuous-type settings, respectively, assuming that reservation utility depends on the agent type - what leads to countervailing incentives and may result in a different solution structure compared to the above classical results. Regarding iii), Rochet and Stole (2003) point out that most of the studies assume the information asymmetry to be one-dimensional, while the agent's hidden characteristics are in many settings inherently multi-dimensional. This may render a natural ordering of the buyer types impossible and the problem solution more involved (see also Schottmüller, 2015). Rochet and Stole (2003) and Rochet and Choné (1998) present in this regard solution methods for discrete and continuous-type settings that allow both for multi-dimensional private information and multiple goods. Their insights indicate that bunching is characteristic for multi-dimensional settings. McAfee and McMillan (1988) propose a generalized single-crossing condition for multi-dimensional multi-product settings, while Figalli et al. (2011) study conditions under which a continuous-type setting leads to a convex program. Regarding iv), Schottmüller 


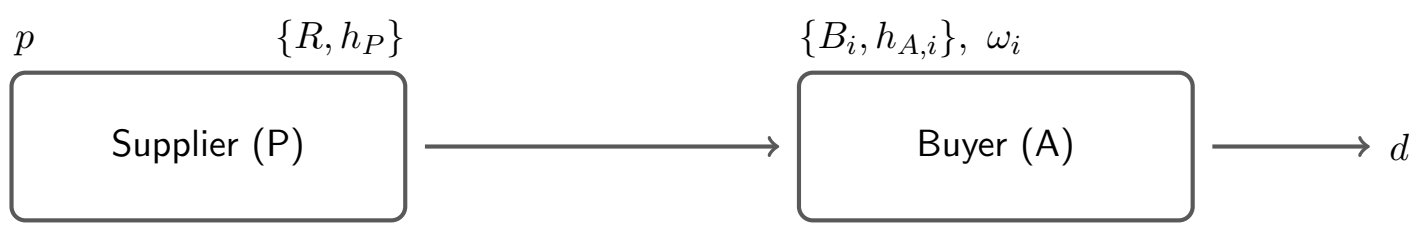

Figure 1: A single-supplier-single-buyer supply chain under consideration

(2015) points out that, although the multi-dimensional agent types can always be enumerated and ordered in some way in a discrete-type setting, the single-crossing property is unlikely to hold. He also indicates other reasons, for which the agent utility may naturally violate the single-crossing condition, even if his hidden information is intrinsically one-dimensional. As a consequence, it is not sufficient to take only local incentive compatibility constraints into account when solving the problem. Araujo and Moreira (2010) and Schottmüller (2015) characterize optimal solutions in a continuous-type setting when the single-crossing condition is violated at most once and show how the optimal solution structure can differ from the classical result. In the next section we turn to discussing the JELS problem with asymmetric information as an adverse selection problem. In Section 4 we will then establish properties of its optimal solutions.

\section{Joint economic lot size problem with asymmetric information}

Consider a supply chain with a single supplier and a single buyer (Figure 1). The buyer faces a deterministic constant demand for a particular product over an infinite horizon and meets the demand from stock that he replenishes by repetitively re-ordering the product from the supplier. Upon receiving the buyer's order, the supplier sets up a production batch, manufactures the order, and ships it to the buyer. Thus, the buyer's order size represents, at the same time, the supplier's manufacturing lot size, as per lot-for-lot assumption (Banerjee, 1986). The supplier aims to negotiate the order size with the buyer, while knowing the buyer's cost structure only probabilistically. Following Sucky (2006), the problem data is comprised on the buyer side (referred to as $A$ ) of:

d deterministic constant demand for the product, in units per time unit,

$B_{i} \quad$ fixed ordering cost in the buyer's possible cost structure no. $i(i=1,2)$,

$h_{A, i} \quad$ unit holding cost per time unit in the buyer's possible cost structure no. $i(i=1,2)$,

$\omega_{i} \quad$ probability for the buyer to have the $i$-th cost structure $\left\{B_{i}, h_{A, i}\right\},\left(\omega_{i}>0, i=1,2\right)$,

and on the supplier side (referred to as $P$ ) of:

$p \quad$ production rate, in units per time unit $(p>d)$,

$R \quad$ fixed setup cost per order,

$h_{P} \quad$ unit holding cost per time unit. 
The individual costs of the parties per time unit turn out then to be respectively the following functions of the order size $x$ (cf. Banerjee, 1986):

$$
\begin{array}{ll}
K_{i}^{A}(x)=B_{i} \cdot \frac{d}{x}+\frac{x}{2} \cdot h_{A, i} & \text { (assuming cost structure no. } i, i=1,2) \\
K^{P}(x)=R \cdot \frac{d}{x}+\frac{x}{2} \cdot \frac{d}{p} h_{P} &
\end{array}
$$

These functions are known to be strictly convex and possess a single minimum (i.e., being unimodal) - provided that all cost parameters involved are positive, what we will assume to hold.

Assuming that the supplier has all of the bargaining power in this supply chain, while the buyer has the decision authority with regard to choosing the order size, the supplier's bargaining problem can thus be stated as an adverse selection problem with the supplier acting as the uninformed principal and the buyer as the privately informed agent. Agent type is represented by the buyer's cost structure $\theta_{i}:=\left\{B_{i}, h_{A, i}\right\}$, and has the associated probability $\omega_{i}\left(i=1,2 ; \theta_{1} \neq \theta_{2}\right)$. The parties need to settle on the terms of contract $(x, z)$, where $x$ is the order size and $z$ is the transfer payment (side payment) from the supplier to the buyer. The buyer's and the supplier's utilities are accordingly:

$$
\begin{aligned}
u\left(\theta_{i}, x, z\right) & =-K_{i}^{A}(x)+z \\
v(x, z) & =-K^{P}(x)-z .
\end{aligned} \quad(i=1,2)
$$

Let further $x_{A, i}^{*}$ denote the buyer's EOQ that minimizes the cost function $K_{i}^{A}(x), i=1,2$. The reservation utility of the buyer with cost structure $\theta_{i}$ (buyer type $i$ ) that he gets if bargaining fails is accordingly $\underline{u}_{i}=-K_{i}^{A}\left(x_{A, i}^{*}\right)$. As discussed in Section 2, the supplier's problem reduces to that of designing a menu (a set) of two offers $\left(x_{1}, z_{1}\right),\left(x_{2}, z_{2}\right)$ to the buyer that solves (Sucky, 2006): ${ }^{3}$

$$
\begin{array}{ll}
\min & \omega_{1} \cdot\left(K^{P}\left(x_{1}\right)+z_{1}\right)+\omega_{2} \cdot\left(K^{P}\left(x_{2}\right)+z_{2}\right) \\
\text { s.t. } & K_{1}^{A}\left(x_{1}\right)-z_{1} \leq K_{1}^{A}\left(x_{A, 1}^{*}\right) \\
& K_{2}^{A}\left(x_{2}\right)-z_{2} \leq K_{2}^{A}\left(x_{A, 2}^{*}\right) \\
& K_{1}^{A}\left(x_{1}\right)-z_{1} \leq K_{1}^{A}\left(x_{2}\right)-z_{2} \\
& K_{2}^{A}\left(x_{2}\right)-z_{2} \leq K_{2}^{A}\left(x_{1}\right)-z_{1} \\
& x_{1}, x_{2}>0
\end{array}
$$

\footnotetext{
${ }^{3}$ In Sucky (2006), problem (1)-(6) additionally involves non-negativity constraints $z_{1}, z_{2} \geq 0$. It is, however, easy to see that constraints (2)-(3) automatically exclude side payments $z_{1}, z_{2}<0$ from consideration due to optimality of order sizes $x_{A, 1}^{*}, x_{A, 2}^{*}$ to the respective buyer types. This allows to omit non-negativities $z_{1}, z_{2} \geq 0$ from (1)-(6), which, in turn, simplifies the analytical solution by avoiding the additional assumption of $z_{1}, z_{2}>0$ (Sucky, 2006).
} 

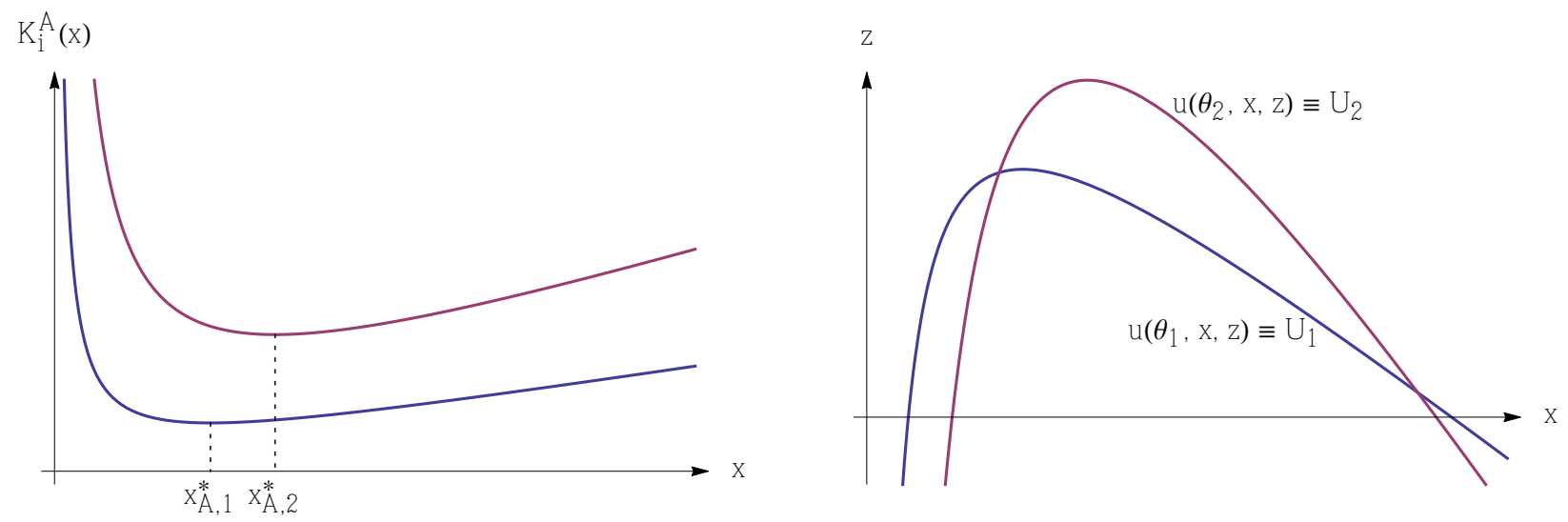

Figure 2: Cost functions of the buyer types (left) and their indifference curves for some given utility levels $U_{1}, U_{2}$ (right) when the buyer's possible cost structures strictly dominate one another in Pareto sense

Note that the above problem is stated in terms of the firms' disutilities, with (2)-(3) representing the individual rationality constraints, and (4)-(5) - the incentive-compatibility constraints.

Problem (1)-(6) obviously complies with assumption i) of Section 2 due to strict convexity of the firms' cost functions. However, assumption ii) does not hold in general because reservation utilities in (2)-(3) are type-dependent. Further, assumption iii) is violated as well, since the buyer's private information is two-dimensional. We shall distinguish in this regard between two cases:

a) Buyer's possible cost structures strictly dominate one another in Pareto sense - i.e., $B_{i}>B_{3-i}$ and $h_{A, i}>h_{A, 3-i}$ holds for some $i \in\{1,2\}$.

b) Buyer's possible cost structures are both weakly Pareto-efficient - i.e., either $B_{1} \leq B_{2}$ and $h_{A, 1} \geq h_{A, 2}$, or $B_{1} \geq B_{2}$ and $h_{A, 1} \leq h_{A, 2}$ holds.

Case a) leads to violation of assumption iv). ${ }^{4}$ Indeed, in this case, the cost curves of the buyer types are situated one above the other, as illustrated in Figure 2 (left). The graph on the right shows that indifference curves can, as a consequence, cross twice, which implies that the two buyer types cannot be discriminated by their marginal rate of substitution between order size and payment. Further, this case proves to satisfy assumption v). Indeed, the buyer types can naturally be arranged in the order of increasing cost $B_{i}$, under which assumption v) holds. Thus, we can distinguish in case a) between more and less efficient buyer types, but cannot do so "at the margin".

On the contrary, case b) satisfies assumption iv) but partly violates assumption v). Figure 3 illustrates this. It is straightforward to verify that the cost curves of the buyer types cross in this case at most once, and so do any two indifference curves. The single-crossing condition of assumption iv) is therefore satisfied. Regarding assumption v), observe that the $\operatorname{costs} K_{1}^{A}(x)$ and

\footnotetext{
${ }^{4}$ We are thankful to an anonymous reviewer for pointing our attention to this.
} 

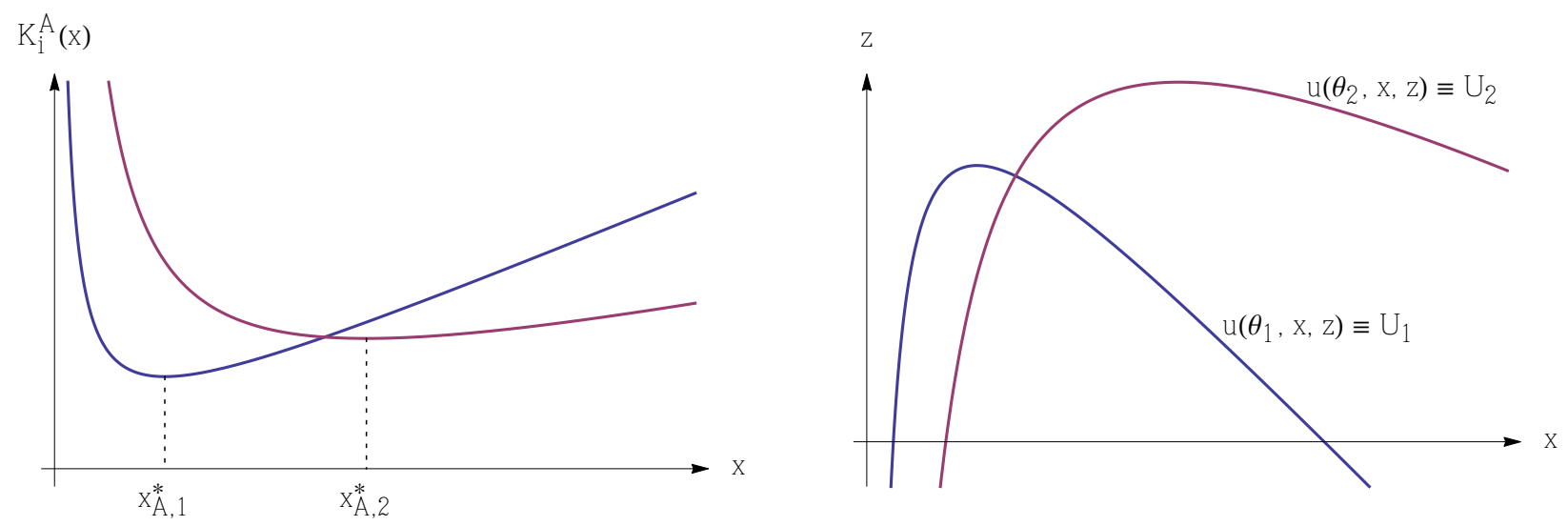

Figure 3: Cost functions of the buyer types (left) and their indifference curves for some given utility levels $U_{1}, U_{2}$ (right) when the buyer's possible cost structures are both Pareto-efficient

$K_{2}^{A}(x)$ of the two buyer types cannot be ordered consistently for all $x$, except when $h_{A, 1}=h_{A, 2}$. Thus, the first part of assumption v) is violated whenever $h_{A, 1} \neq h_{A, 2}$. Without loss of generality, assume that $B_{1} \leq B_{2}$ and $h_{A, 1} \geq h_{A, 2}$ holds (see Figure 3 ). Then $K_{i}^{A^{\prime \prime}}(x)$ is non-decreasing in $i$, what satisfies the second part of assumption v). ${ }^{5}$ To summarize, we cannot consistently distinguish in case b) between the more and less efficient buyer types, but can do so "at the margin".

Thus, problem (1)-(6) in general violates assumptions ii), iii) and either assumption iv) or (partly) assumption v). To the best of our knowledge, none of the existing works has studied adverse selection problems with these properties in their generality. As the classical solution approach outlined in Section 2 cannot be used, we follow Sucky (2006) in solving problem (1)-(6) in its general form by means of Karush-Kuhn-Tucker (KKT) conditions (Bazaraa et al., 2006).

KKT conditions and problem constraints (2)-(5) jointly comprise a system of equations and inequalities (A.1)-(A.8) given in Appendix A. A set of offers that satisfies (A.1)-(A.8) for some nonnegative values of Lagrange multipliers $\lambda_{1}, \lambda_{2}, \mu_{1}, \mu_{2}$ is called a KKT solution of problem (1)(6), or its stationary point. By distinguishing between a zero and a positive value to be taken on by each of the four Lagrange multipliers, one obtains $2^{4}=16$ distinct cases, of which only eight prove to be feasible (Sucky, 2006, p. 531). Solving system (A.1)-(A.8) analytically in its general form leads therefore to eight generic types of KKT solutions.

We collate all types of KKT solutions from 1 to 8 in Table 1 . Note that each type is represented by a closed-form expression of one or more candidate sets of offers as well as associated feasibility and necessary optimality conditions. If a candidate set of offers satisfies the respective conditions then, by construction, it qualifies as a KKT solution of problem (1)-(6). We refer the reader to Sucky (2006, Section 5.2) for derivation of KKT solutions of types 1 to 6 .

\footnotetext{
${ }^{5}$ Regarding assumption iv), note that $K_{i}^{A^{\prime}}(x)$ is then strictly decreasing in $i$. See in this regard footnote 2.
} 


\begin{tabular}{|c|c|c|c|c|}
\hline Type & Order sizes & Side payments & Feasibility conditions & Necessary optimality conditions \\
\hline \multirow[t]{2}{*}{1} & $x_{1}=\mathrm{JELS}_{1}$ & $z_{1}=K_{1}^{A}\left(x_{1}\right)-K_{1}^{A}\left(x_{A, 1}^{*}\right)$ & $K_{2}^{A}\left(x_{1}\right)-z_{1} \geq K_{2}^{A}\left(x_{A, 2}^{*}\right)$ & \\
\hline & $x_{2}=\mathrm{JELS}_{2}$ & $z_{2}=K_{2}^{A}\left(x_{2}\right)-K_{2}^{A}\left(x_{A, 2}^{*}\right)$ & $K_{1}^{A}\left(x_{2}\right)-z_{2} \geq K_{1}^{A}\left(x_{A, 1}^{*}\right)$ & \\
\hline \multirow[t]{2}{*}{2} & $x_{1}=\sqrt{2 d \cdot \frac{\omega_{1} S_{1}+\omega_{2} \bar{B}}{\omega_{1}+\omega_{\bar{h}}}}$ & $z_{1}=K_{1}^{A}\left(x_{1}\right)-K_{1}^{A}\left(x_{A, 1}^{*}\right)$ & $K_{2}^{A}\left(x_{1}\right)-z_{1} \leq K_{2}^{A}\left(x_{A, 2}^{*}\right)$ & \\
\hline & $x_{2}=\mathrm{JELS}_{2} \omega_{1} H_{1}+\omega_{2} h_{A}$ & $z_{2}=K_{2}^{A}\left(x_{2}\right)-K_{2}^{A}\left(x_{1}\right)+z_{1}$ & $K_{1}^{A}\left(x_{2}\right)-z_{2} \geq K_{1}^{A}\left(x_{A, 1}^{*}\right)$ & \\
\hline \multirow[t]{2}{*}{3} & $x_{1}=\mathrm{JELS}_{1}$ & $z_{1}=K^{A}\left(r_{1}\right)-K$ & $K^{A}\left(r_{1}\right)-\gamma_{1}>K^{A}\left(r^{*}\right)$ & \\
\hline & $x_{2}=\sqrt{2 d \cdot \frac{\omega_{2} S_{2}-\omega_{1} \bar{B}}{\omega_{2} H_{2}-\omega_{1} \bar{h}_{A}}}$ & $z_{2}=K_{2}^{A}\left(x_{2}\right)-K_{2}^{A}\left(x_{A, 2}^{*}\right)$ & $K_{1}^{A}\left(x_{2}\right)-z_{2} \leq K_{1}^{A}\left(x_{A, 1}^{*}\right)$ & \\
\hline \multirow[t]{2}{*}{$4^{\dagger}$} & $x_{1}=\mathrm{JELS}_{1}$ & $z_{1}=K_{1}^{A}\left(x_{1}\right)-K_{1}^{A}\left(x_{A, 1}^{*}\right)$ & & $x_{A, 1}^{*}=x_{A, 2}^{*}, x_{2}=\mathrm{JELS}_{2}$ \\
\hline & $x_{2_{1,2}}=\sqrt{2 d} \cdot \frac{\sqrt{B_{1}} \pm \sqrt{B_{2}}}{\sqrt{h_{A, 1}} \pm \sqrt{h_{A, 2}}}$ & $z_{2}=K_{2}^{A}\left(x_{2}\right)-K_{2}^{A}\left(x_{A, 2}^{*}\right)$ & $K_{2}^{A}\left(x_{1}\right)-z_{1} \geq K_{2}^{A}\left(x_{A, 2}^{A}\right)$ & $0 \leq \mathcal{M}_{1}\left(x_{2}\right) \leq \omega_{1} / \omega_{2}$ \\
\hline \multirow[t]{2}{*}{$5^{\dagger}$} & $x_{1_{1,2}}=\sqrt{2 d} \cdot \frac{\sqrt{B_{1}} \pm \sqrt{B_{2}}}{\sqrt{h}+\sqrt{h}}$ & $z_{1}=K_{1}^{A}\left(x_{1}\right)-K_{1}^{A}\left(x_{A, 1}^{*}\right)$ & $K^{A}{ }_{(}$ & $x_{A, 1}^{*}=x_{A, 2}^{*}, x_{1}=\mathrm{JELS}_{1}$ \\
\hline & $x_{2}=\mathrm{JELS}_{2}$ & $z_{2}=K_{2}^{A}\left(x_{2}\right)-K_{2}^{A}\left(x_{A, 2}^{*}\right)$ & $\Lambda_{1}\left(x_{2}\right)-z_{2}=\Lambda_{1}\left(x_{A, 1}\right)$ & $0 \leq \mathcal{M}_{2}\left(x_{1}\right) \leq \omega_{2} / \omega_{1}$ \\
\hline \multirow{2}{*}{$6^{\dagger}$} & $\overline{2 d} \cdot \frac{\sqrt{B_{1}} \pm \sqrt{B_{2}}}{2}$ & $z_{1}=K_{1}^{A}\left(x_{1}\right)-K_{1}^{A}\left(x_{A, 1}^{*}\right)$ & & $x_{A, 1}^{*}=x_{A, 2}^{*}, x_{1}=\mathrm{JELS}_{1}, x_{2}=\mathrm{JELS}_{2}$ \\
\hline & $\sqrt{h_{A, 1}} \pm \sqrt{h_{A, 2}}$ & $z_{2}=K_{2}^{A}\left(x_{2}\right)-K_{2}^{A}\left(x_{A, 2}^{*}\right)$ & & $\mathcal{M}_{1}\left(x_{2}\right), \mathcal{M}_{2}\left(x_{1}\right) \geq 0,-\omega_{2} \leq \omega_{2} \mathcal{M}_{1}\left(x_{2}\right)-\omega_{1} \mathcal{M}_{2}\left(x_{1}\right) \leq \omega_{1}$ \\
\hline \multirow[t]{2}{*}{$7 \ddagger$} & $x_{1}=x_{2}=\sqrt{\mathcal{A}}$ & $z_{1}-K^{A}\left(r_{1}\right)-K^{A}\left(r^{*}\right)$ & & $x_{1}=x_{2}=\mathrm{JELS}_{2}$ \\
\hline & $x_{1}=\sqrt{2 d \cdot \frac{\omega_{1} S_{2}+\omega_{2} H_{2} \cdot \bar{B} / \bar{h}_{A}}{\omega_{1} H_{2}+\omega_{2} S_{2} \cdot \bar{h}_{A} / \bar{B}}}, \quad x_{2}=\frac{\mathcal{A}}{x_{1}}$ & $z_{2}=K_{2}^{A}\left(x_{2}\right)-K_{2}^{A}\left(x_{A, 2}^{*}\right)$ & $K_{1}^{A}\left(x_{2}\right)-z_{2} \leq K_{1}^{A}\left(x_{A, 1}^{*}\right)$ & $\mathcal{M}_{2}\left(x_{2}\right) \leq-1 / \omega_{2}$ \\
\hline \multirow[t]{2}{*}{$8^{\ddagger}$} & $x_{1}=x_{2}=\sqrt{\mathcal{A}}$ & $z_{-}-K^{A}\left(r_{1}\right)-k$ & & $x_{1}=x_{2}=\mathrm{JELS}_{1}$ \\
\hline & $x_{1}=\sqrt{2 d \cdot \frac{\omega_{1} S_{1}+\omega_{2} H_{1} \cdot \bar{B} / \bar{h}_{A}}{\omega_{1} H_{1}+\omega_{2} S_{1} \cdot \bar{h}} / \bar{B}}, \quad x_{2}=\frac{\mathcal{A}}{x_{1}}$ & $z_{2}=K_{1}^{A}\left(x_{2}\right)-K_{1}^{A}\left(x_{A, 1}^{*}\right)$ & $K_{2}^{A}\left(x_{1}\right)-z_{1} \leq K_{2}^{A}\left(x_{A, 2}^{*}\right)$ & $\mathcal{M}_{1}\left(x_{1}\right) \leq-1 / \omega_{1}$ \\
\hline
\end{tabular}

${ }_{\dagger}^{\dagger}$ Expression of the set of offers has been simplified and its necessary optimality conditions refined. $x_{i_{1,2}}$ expresses two possible order sizes for offering to the buyer type $i \in\{1,2\}$; $x_{1,2,2}$ respectively expresses two possible order sizes for offering to each buyer type $i=1,2 . \quad{ }^{\ddagger}$ Set of offers is newly derived in this work. $\quad$ ¥¥ In addition, $x_{1}, x_{2}>0$ must hold for feasibility. A set of offers satisfying the feasibility and necessary optimality conditions is a KKT solution of the problem. A horizontal separator line stands for the logical 'or'.

Notation: $(i=1,2)$
$S_{i}=R+B_{i}$
$\bar{B}=B_{1}-B_{2}$
$x_{A, i}^{*}=\sqrt{\frac{2 d B_{i}}{h_{A, i}}}$
$H_{i}=\frac{d}{p} h_{P}+h_{A, i}$
$\bar{h}_{A}=h_{A, 1}-h_{A, 2}$
$\operatorname{JELS}_{i}=\sqrt{\frac{2 d S_{i}}{H_{i}}}$
$\mathcal{M}_{1}(x)=\frac{H_{2} x^{2}-2 d S_{2}}{\bar{h}_{A} x^{2}-2 d \bar{B}}$
$\mathcal{A}=2 d \cdot \frac{\bar{B}}{\bar{h}_{A}}$
$\mathcal{M}_{2}(x)=-\frac{H_{1} x^{2}-2 d S_{1}}{\bar{h}_{A} x^{2}-2 d \bar{B}}$ 
In the present work, we contribute to these results by:

1) refining KKT solutions of types $4-6$;

2) deriving KKT solutions of types 7-8.

We provide specific details in Appendix B and Appendix C, respectively. These results simplify problem solution and ease its analysis that we conduct below.

In the next section we turn to studying the properties of KKT solutions for the purpose of determining optimal solutions of the problem. To simplify the subsequent exposition, we will use the notation $\mathrm{JELS}_{i}$ to refer to the joint economic lot size associated with the buyer's cost structure no. $i$. By definition, this is the order size that minimizes the total costs $K^{P}(x)+K_{i}^{A}(x)$ of the supplier and the buyer and has therefore the following expression (cf. Banerjee, 1986; Sucky, 2006):

$$
\mathrm{JELS}_{i}=\sqrt{2 d \cdot \frac{B_{i}+R}{h_{A, i}+\frac{d}{p} h_{P}}}, \quad i=1,2 .
$$

\section{Properties of optimal solutions}

As indicated in Section 3, Table 1 collates all types of KKT solutions from 1 to 8. The indicated sets of offers represent candidate solutions; they qualify as KKT solutions if they satisfy the respective feasibility and necessary optimality conditions. We should stress at this point that a KKT solution still does not need to represent an optimal solution to problem (1)-(6) because the problem proves to be non-convex - despite of convexity of the functions involved. Indeed, by rewriting the problem in a standard form

$$
\begin{array}{cl}
\min & \omega_{1} \cdot\left(K^{P}\left(x_{1}\right)+z_{1}\right)+\omega_{2} \cdot\left(K^{P}\left(x_{2}\right)+z_{2}\right) \\
\text { s.t. } & K_{1}^{A}\left(x_{1}\right)-z_{1}-K_{1}^{A}\left(x_{A, 1}^{*}\right) \leq 0 \\
& K_{2}^{A}\left(x_{2}\right)-z_{2}-K_{2}^{A}\left(x_{A, 2}^{*}\right) \leq 0 \\
& K_{1}^{A}\left(x_{1}\right)-z_{1}-K_{1}^{A}\left(x_{2}\right)+z_{2} \leq 0 \\
& K_{2}^{A}\left(x_{2}\right)-z_{2}-K_{2}^{A}\left(x_{1}\right)+z_{1} \leq 0
\end{array}
$$

with $x_{1}, x_{2}>0$, we can see that objective function (8) and the left-hand sides in (9)-(10) are indeed convex in $x_{1}, z_{1}, x_{2}, z_{2}$, whereas the left-hand sides in (11)-(12) are not, in particular because $-K_{1}^{A}\left(x_{2}\right)$ and $-K_{2}^{A}\left(x_{1}\right)$ are strictly concave functions of their arguments. An example in Table 2 illustrates a situation in which four sets of offers (of types 3,4 and 7 ) satisfy the respective feasibility and necessary optimality conditions and therefore qualify as KKT solutions. Only one of these solutions is optimal. This example shows that KKT conditions indeed do not need to be sufficient 


\begin{tabular}{llll}
\hline Supplier & Buyer: cost structure no. 1 & cost structure no. 2 & Objective value \\
\hline$R=120, h_{P}=10$ & $\begin{array}{l}B_{1}=120, h_{A, 1}=100, \omega_{1}=0.7 \\
d=10000\end{array}$ & $B_{2}=30, h_{A, 2}=50, \omega_{2}=0.3$ & \\
$p=15000$ & $\left(x_{1}, z_{1}\right) \approx(212.1,886.7)$ & $\left(x_{2}, z_{2}\right) \approx(141.4,179.6)$ & 7816.4 \\
\hline Set of offers of type $3:$ & $\left(x_{1}, z_{1}\right) \approx(212.1,771.5)$ & $\left(x_{2}, z_{2}\right) \approx(136.1,129.8)$ & 7814.5 \\
Set of offers of type 4 $(\mathrm{a}):$ & $\left(x_{2}, z_{2}\right) \approx(264.5,2268.7)$ & 7301.2 \\
Set of offers of type $4(\mathrm{~b}):$ & $\left(x_{1}, z_{1}\right) \approx(212.1,771.5)$ & $\left(x_{2}, z_{2}\right) \approx(175.8,624.1)$ & 7765.7 \\
Set of offers of type $7:$ & $\left(x_{1}, z_{1}\right) \approx(204.8,1107.4)$ & &
\end{tabular}

Table 2: Example of multiple sets of offers satisfying the respective feasibility and necessary optimality conditions

for optimality. Nonetheless, there holds the following

Proposition 1. KKT conditions (A.1)-(A.8) are necessary for local (and hence global) optimality.

Proof. See Appendix G.

Proposition 1 implies that optimal solutions of problem (1)-(6) are captured by those sets of offers of types 1 to 8 that satisfy the respective feasibility and necessary optimality conditions, as given in Table 1. Thus in general, all of these sets of offers need to be checked against their feasibility and necessary optimality conditions in order to detect KKT solutions of the given problem instance, and then objective function (8) has to be evaluated at each KKT solution to determine an optimal one. Note that in the example given in Table 2, picking just the first set of offers on the list that satisfies the feasibility and necessary optimality conditions (i.e., set of offers of type 3) would have led to a non-optimal decision represented by a KKT solution with the worst objective value.

The following result shows that the search for optimal solutions can nevertheless be restricted to certain types of KKT solutions only, because any other KKT solution is guaranteed to be suboptimal. To be specific, observe that Table 1 contains expressions of 15 candidate solutions to problem (1)-(6) that are divided into eight types. We can index all candidate solutions as

$$
S:=\{1,2,3,4 \mathrm{a}-\mathrm{b}, 5 \mathrm{a}-\mathrm{b}, 6 \mathrm{a}-\mathrm{d}, 7 \mathrm{a}-\mathrm{b}, 8 \mathrm{a}-\mathrm{b}\}
$$

where solutions of the same type are sub-indexed with literals. ${ }^{6}$ Then, there holds the following

Proposition 2. In an optimal set of offers, $x_{i}=\operatorname{JELS}_{i}$ holds for at least one $i \in\{1,2\}$.

Proof. See Appendix H.

\footnotetext{
${ }^{6}$ Sub-indexing of solutions of types 4 to 6 follows the lexicographical order of lot sizes $x_{i_{1,2}}$ that determine the specific set of offers in Table $1(i \in\{1,2\})$. E.g., of the two possible expressions for the order size $x_{2}$ in a set of offers of type 4 , solution $4 \mathrm{a}$ involves order size $x_{2_{1}}$, while solution $4 \mathrm{~b}$ involves order size $x_{2_{2}}$. Sub-indexing of solutions of types 7 and 8 follows the order of their definition in Table 1.
} 
An immediate consequence of the proof of Proposition 2 is the following

Corollary 1. An optimal set of offers from $\{6 \mathrm{a}-\mathrm{d}, 7 \mathrm{~b}, 8 \mathrm{~b}\}$ necessarily coincides with some other set of offers from

$$
S^{*}=\{1,2,3,4 \mathrm{a}-\mathrm{b}, 5 \mathrm{a}-\mathrm{b}, 7 \mathrm{a}, 8 \mathrm{a}\} .
$$

We can for this reason disregard candidate solutions $6 \mathrm{a}-\mathrm{d}, 7 \mathrm{~b}, 8 \mathrm{~b}$ when determining optimal solutions, and restrict our attention only to those contained in $S^{*}$. Taking into account Observation 1 in Appendix C, the above result can further be strengthened as follows.

Corollary 2. A problem instance with $\mathrm{JELS}_{1} \neq \mathrm{JELS}_{2}$ has all its optimal solutions among candidate solutions of types 1 to 5 .

Proposition 2 and Corollaries 1-2 lead us to establishing the following properties of optimal sets of offers.

1. By Proposition 2, at least one of the order sizes in the menu is first-best, being represented by the respective joint economic lot size. Thus, there is no distortion from the first-best for at least one of the buyer types. This is in line with the classical result discussed in Section 2.

2. When $\mathrm{JELS}_{1} \neq \mathrm{JELS}_{2}$, i.e. when the first-best quantities depend on the buyer type, Corollary 2 implies that there are five generic types of optimal menus. These five generic types can be described as follows: type 1 - first-best quantities, both individual rationality (IR) constraints bind, while the incentive compatibility (IC) constraints do not; types 2 and 3 one IR constraint and one IC constraint bind; types 4 and 5 - both IR constraints and one IC constraint bind. This result is in line with the existence of five possible menu types derived by Kerschbamer and Maderner (1998) for the adverse selection problem with countervailing incentives under more restrictive assumptions (among which is a strict monotonicity of utility functions in quantity, and the single-crossing condition). Our result thus generalizes theirs.

3. Further, when $\mathrm{JELS}_{1} \neq \mathrm{JELS}_{2}$, bunching does not occur. This follows from the expressions of the menus of types 1-5 in Table 1. Indeed, it is immediately seen that the order sizes in the menu of type 1 cannot coincide; the same can be easily derived from the expressions of order sizes in the menus of types 2 and 3 . The same conclusion can be derived for the menus of types 4 and 5 from their necessary optimality conditions. This generalizes the result of Kerschbamer and Maderner (1998) as well.

4. When $\mathrm{JELS}_{1}=\mathrm{JELS}_{2}$, we have that the first-best quantity is the same for both buyer types. Further, it follows from the definition of joint economic lot sizes in (7) that the cost 
structures of the buyer types must strictly dominate one another in Pareto sense. Paretodominance enables candidate solutions of type 7 and 8 , that are undefined otherwise. When $\mathrm{JELS}_{1}=\mathrm{JELS}_{2}$, either menu 7a or menu 8a proves to be a KKT solution (see Table 1). Furthermore, menu 7a (resp. 8a) coincides with the menu of type 3 (resp. 2) when the latter is defined, and supplants it otherwise (see Appendix F). Hence, the conclusion about five generic menu types sustains in this case as well.

5. When $\mathrm{JELS}_{1}=\mathrm{JELS}_{2}$, we have a possibility of bunching. Bunching occurs namely when a KKT solution of type $7 \mathrm{a}$ or $8 \mathrm{a}$ proves to be optimal. This possibility represents a marked contrast to the classical result of Section 2 and the results of Kerschbamer and Maderner (1998). We elaborate in Proposition 3 below on the conditions under which bunching takes place.

To summarize, the following holds true: in an optimal set of offers, there is no distortion for at least one buyer type; there exist five generic types of optimal menus; bunching can only occur when the first-best quantities coincide. The following proposition gives precise conditions for the occurrence of bunching. To simplify the subsequent exposition, let

$$
S_{i}=R+B_{i} \quad \text { and } \quad H_{i}=\frac{d}{p} h_{P}+h_{A, i} \quad(i=1,2) .
$$

Furthermore, let ELS $\equiv x_{P}^{*}=\sqrt{2 p R / h_{P}}$ represent the supplier's economic lot size and $\mathrm{EOQ}_{i} \equiv$ $x_{A, i}^{*}=\sqrt{2 d B_{i} / h_{A, i}}-$ the economic order quantity of the buyer type $i$.

Proposition 3. Let $\mathrm{JELS}_{1}=\mathrm{JELS}_{2}$ hold. Then the buyer's possible cost structures strictly Pareto-dominate one another. Without loss of generality, assume that $B_{1}>B_{2}$ and $h_{A, 1}>h_{A, 2}$. Then the following is true:

i) If $\mathrm{EOQ}_{1}=\mathrm{EOQ}_{2}=\mathrm{ELS}$ holds then the first-best outcome is realised without bargaining.

ii) Otherwise, the supplier's optimal set of offers entails bunching if and only if $\omega_{1} \leq H_{2} / H_{1}$.

Proof. See Appendix I.

Assertion i) of the proposition is obvious: when all individually optimal order sizes coincide, they represent the first-best outcome per se, and therefore there is no need for bargaining. Assertion ii) is illustrated in Figure 4 by referring to the problem instance given in Table C.3 of Appendix C. This instance has $\mathrm{JELS}_{1}=\mathrm{JELS}_{2}$. Figure 4 shows the cost curves $K^{P}(x)$ and $K_{i}^{A}(x)$, the economic order quantities $\mathrm{EOQ}_{i}$, and the joint economic lot sizes $\operatorname{JELS}_{i}(i=1,2)$. We can see on the figure that the supplier's costs would be reduced if the buyer adopted the order size $\mathrm{JELS}_{1}=\mathrm{JELS}_{2}$ 


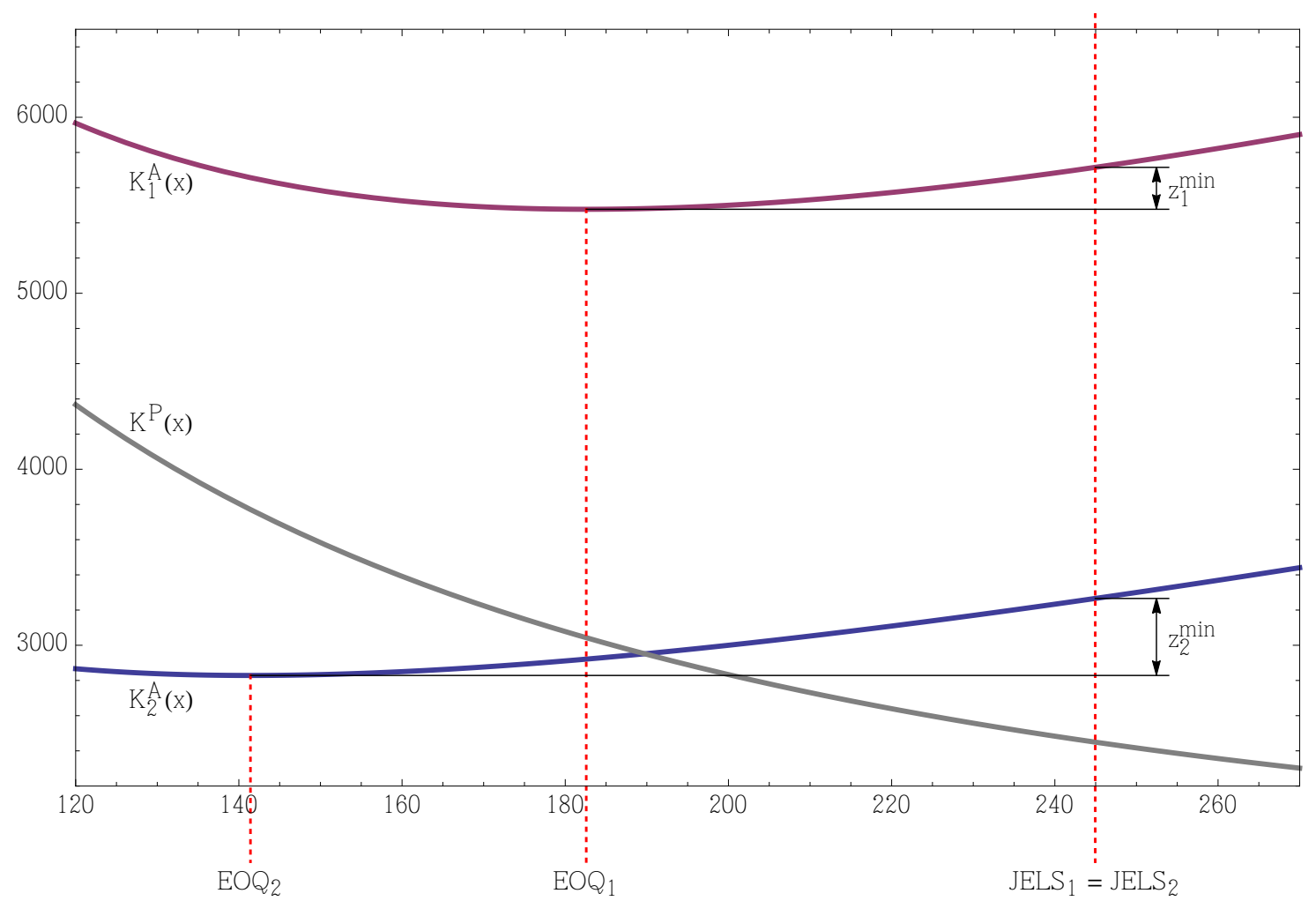

Figure 4: Emergence of the optimal set of offers 7a in the example of Table C.3 (see Appendix C)

instead of his economic order quantity $\operatorname{EOQ}_{i}(i=1,2)$. In order to entice the buyer to do so, the supplier can offer him a side payment $z_{i}$ as a compensation that should thus be at least

$$
z_{i}^{\min }:=K_{i}^{A}\left(\mathrm{JELS}_{i}\right)-K_{i}^{A}\left(\mathrm{EOQ}_{i}\right)
$$

Figure 4 illustrates the emergence of the side payments $z_{1}^{\min }$ and $z_{2}^{\min }$. Note that in either case, the supplier's cost reduction more than offsets the side payment; it is also known that her respective net benefit is biggest when the buyer type $i(i=1,2)$ adopts exactly the order size $\operatorname{JELS}_{i}$ and receives the minimum required compensation. In the presence of two buyer types, the supplier prepares a menu of two respective offers; given that $\mathrm{JELS}_{1}=\mathrm{JELS}_{2}$, both offers contain an identical order size. Thus regardless of the buyer's true cost structure, he will obviously pick the offer with the bigger side payment. Hence the supplier can equivalently design a menu of two identical offers that both feature the bigger of the two possible compensations $z_{1,2}^{\min }$ (what is equivalent to satisfying the incentive compatibility constraints (4)-(5)). As a result, the buyer type requiring actually a lower compensation (buyer type 1 in Figure 4) is going to be overpaid, and this happens with probability $\omega_{1}$. Proposition 3 states that if this probability is not too high - specifically, not above the threshold $H_{2} / H_{1}$ - then it will be optimal for the supplier to adopt the set of offers in question (menu $7 \mathrm{a}$ in the given example), which entails bunching. Otherwise, if $\omega_{1}$ exceeds the threshold, 
it is optimal for the supplier to hedge by adopting a different set of offers (one of type 4) — that intends the joint economic lot size for the buyer type 1, and a different lot size for the buyer type 2 .

The proof of Proposition 3 shows that if $\omega_{1}$ is exactly at the threshold $H_{2} / H_{1}$ (as in the example in Table C.3), the problem has multiple global optima - one represented by menu 7a, and two others - by sets of offers of type 4. Thus, the supplier is indifferent between these three menus; we adopt the convention that in this case, she offers a menu that entails a greater efficiency (in the expectation) - that is, menu 7a with the first-best quantity. Hence the assertion ii) of the proposition: that bunching (via menu 7a) occurs if and only if condition $\omega_{1} \leq H_{2} / H_{1}$ holds.

It is worth noting that sets of offers of type 4 and 7 a do not depend on $\omega_{1}, \omega_{2}$ (see Table 1), and, thus, lack of bunching when the said condition does not hold is solely due to sets of offers of type 4 turning up as KKT solutions and the comparison between the objective values at KKT solutions in their favor. Hence condition $\omega_{1}>H_{2} / H_{1}$ is similar to the monotonicity condition involving the hazard rate of the probability distribution with more than two agent types (see Section 2). ${ }^{7}$ Further, note that, since the equality JELS $1=$ JELS $_{2}$ implies Pareto-dominance among the buyer's possible cost structures, the single-crossing condition does not hold (see Section 3); conversely, under single-crossing, bunching does not emerge, which is in line with the classical results (cf. Section 2).

\section{Optimality of KKT solutions under Pareto-efficiency of the buyer's cost structures}

Although Proposition 2 and Corollaries 1, 2 narrow down the number of candidate solutions to be evaluated using the analytical solution approach, this still, in general, does not obviate the need to deal with multiple KKT solutions even when seeking to find just any optimal solution.

If the problem is being solved by means of numerical optimization techniques, e.g. with a general-purpose solver, then, depending on the choice of the starting point, the algorithm may end up at a KKT solution that does not necessarily represent a global minimum. ${ }^{8}$ Our numerical experience confirms this (we in particular experimented with fmincon solver from matlab's optimization toolbox, version 5.1, and Excel ${ }^{\circledR}$ Solver 2010, using each solver's default settings).

It becomes therefore of interest to investigate the question whether it is possible to establish a condition that would guarantee that a KKT solution of the problem represents its globally optimal solution. When pursuing the analytical approach to solving the problem, such a condition would allow to pick just the first set of offers from the set $S^{*}$ that satisfies the respective feasibility and

\footnotetext{
${ }^{7}$ We are thankful to an anonymous reviewer for pointing us to this.

${ }^{8}$ Neither should it represent a local minimum (cf. Chap. 10 in Bazaraa et al., 2006; Morrow, 2011). Using secondorder sufficient conditions (see e.g. Bazaraa et al., 2006, Theorem 4.4.2) and the results derived in Section D.3 of Appendix D, it can still be shown that non-optimal KKT solutions represented by sets of offers of types 4, 5 and 6 must represent local minima of the problem, provided that the Lagrange multipliers that were assumed to be positive for the respective set of offers (Sucky, 2006, Table 5) have strictly positive values.
} 
necessary optimality conditions as per Table 1 . When pursuing a numerical approach to solving the problem, this condition would assure that the numerical solver that converges to a KKT solution, obtains an optimal solution. In this section we establish such a condition.

To this end, it is instructive to illustrate the emergence of multiple KKT solutions by referring here once again to the problem instance given in Table 2 of Section 4. For illustrative purposes, we will restrict our attention to those feasible solutions of problem (1)-(6), at which individual rationality constraints (2)-(3) are binding, so that the following holds:

$$
z_{1}=K_{1}^{A}\left(x_{1}\right)-K_{1}^{A}\left(x_{A, 1}^{*}\right), \quad z_{2}=K_{2}^{A}\left(x_{2}\right)-K_{2}^{A}\left(x_{A, 2}^{*}\right) .
$$

This allows us to eliminate variables $z_{1}, z_{2}$ and resort to the following surrogate problem in place of the original one (cf. eq. (A.20)-(A.22) in Sucky, 2006):

$$
\begin{array}{cc}
\min & \omega_{1} \cdot\left(K^{P}\left(x_{1}\right)+K_{1}^{A}\left(x_{1}\right)-K_{1}^{A}\left(x_{A, 1}^{*}\right)\right)+\omega_{2} \cdot\left(K^{P}\left(x_{2}\right)+K_{2}^{A}\left(x_{2}\right)-K_{2}^{A}\left(x_{A, 2}^{*}\right)\right) \\
\text { s.t. } & -K_{1}^{A}\left(x_{2}\right)+K_{2}^{A}\left(x_{2}\right)+K_{1}^{A}\left(x_{A, 1}^{*}\right)-K_{2}^{A}\left(x_{A, 2}^{*}\right) \leq 0 \\
& K_{1}^{A}\left(x_{1}\right)-K_{2}^{A}\left(x_{1}\right)-K_{1}^{A}\left(x_{A, 1}^{*}\right)+K_{2}^{A}\left(x_{A, 2}^{*}\right) \leq 0
\end{array}
$$

where $x_{1}, x_{2}>0$. Figure 5 depicts the graph of objective function (14) over the feasible region defined by (15), (16). As one can see, the feasible region consists of two disjoint parts, which gives rise to two distinct local minima. The reason is that one of the buyer's possible cost structures in Table 2 strictly dominates the other in Pareto sense: $B_{1}>B_{2}$ and $h_{A, 1}>h_{A, 2}$, so that the left-hand side in (15), expressing as

$$
\left(B_{2}-B_{1}\right) \cdot \frac{d}{x_{2}}+\frac{x_{2}}{2} \cdot\left(h_{A, 2}-h_{A, 1}\right)+10014.7,
$$

is strictly concave and unimodal, see Figure 6. As a result, (15) is satisfied on two disjoint intervals, which makes the feasible region of (14)-(16) non-convex and leads to existence of two local minima.

Note further that the left-hand sides of constraints (15) and (16) represent univariate functions that are just additive inverses of one another, as Figure 6 illustrates. As a result, a strict Pareto dominance among the buyer's possible cost structures always makes exactly one left-hand side in (15)-(16) strictly concave and unimodal, and leads to multiple local minima.

In contrast, if the buyer's cost structures do not strictly dominate one another in Pareto sense and therefore happen to be weakly Pareto-efficient, then the left-hand sides in (15)-(16) both represent monotone functions. Figure 7 illustrates this for the problem instance obtained from the one given in Table 2 by interchanging the values of parameters $B_{1}$ and $B_{2}$. The consequence is that 


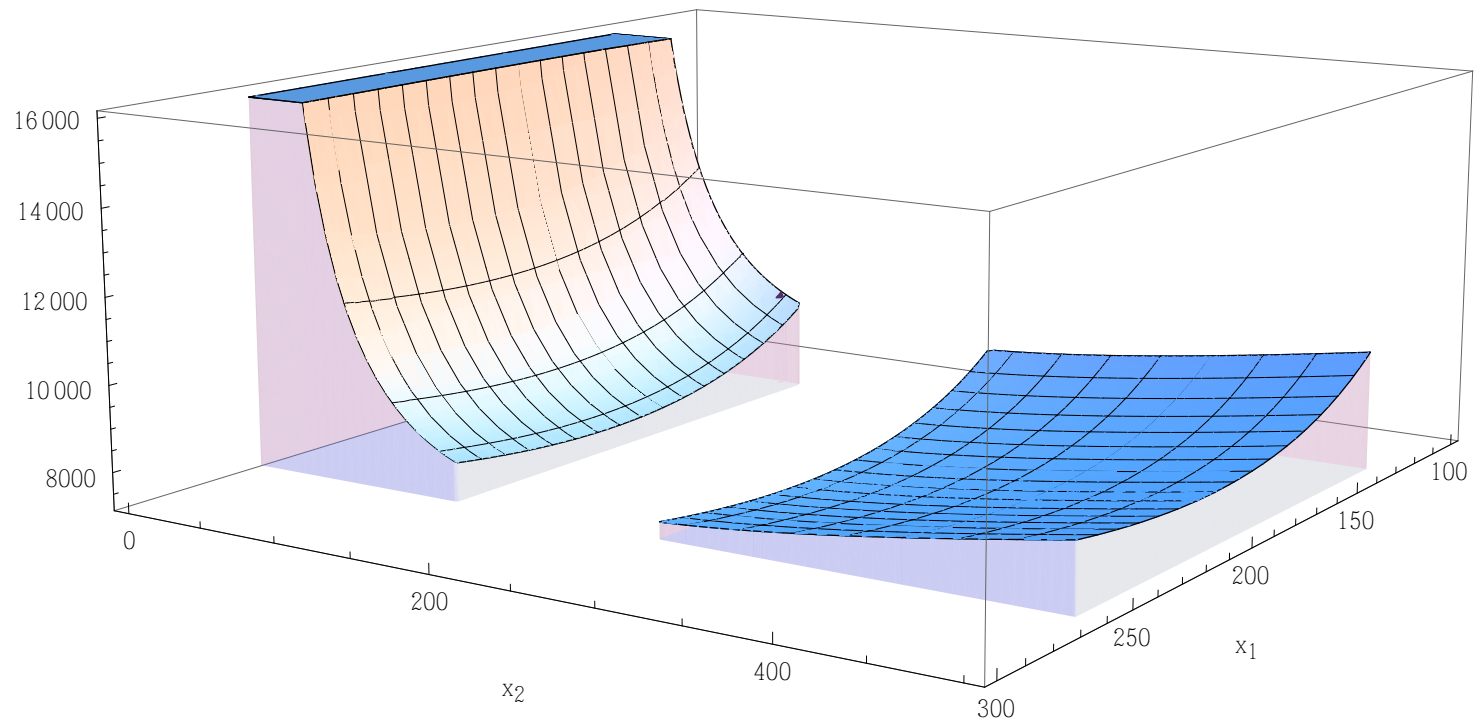

Figure 5: Objective function of the surrogate problem (14)-(16) associated with the example in Table 2
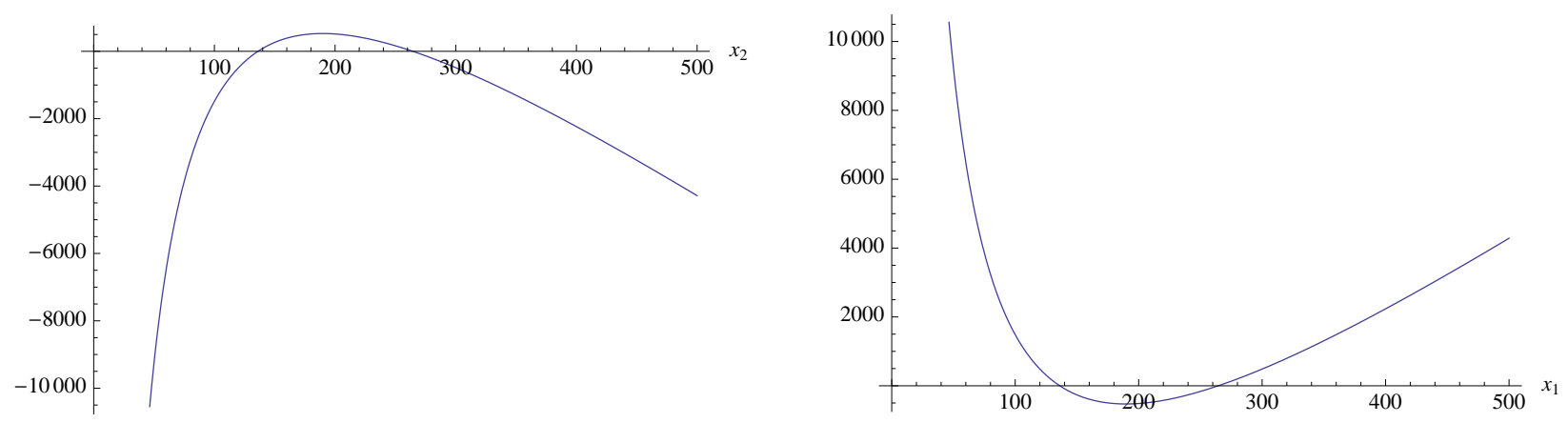

Figure 6: Left-hand sides of surrogate constraints (15) (left) and (16) (right) in the problem instance of Table 2
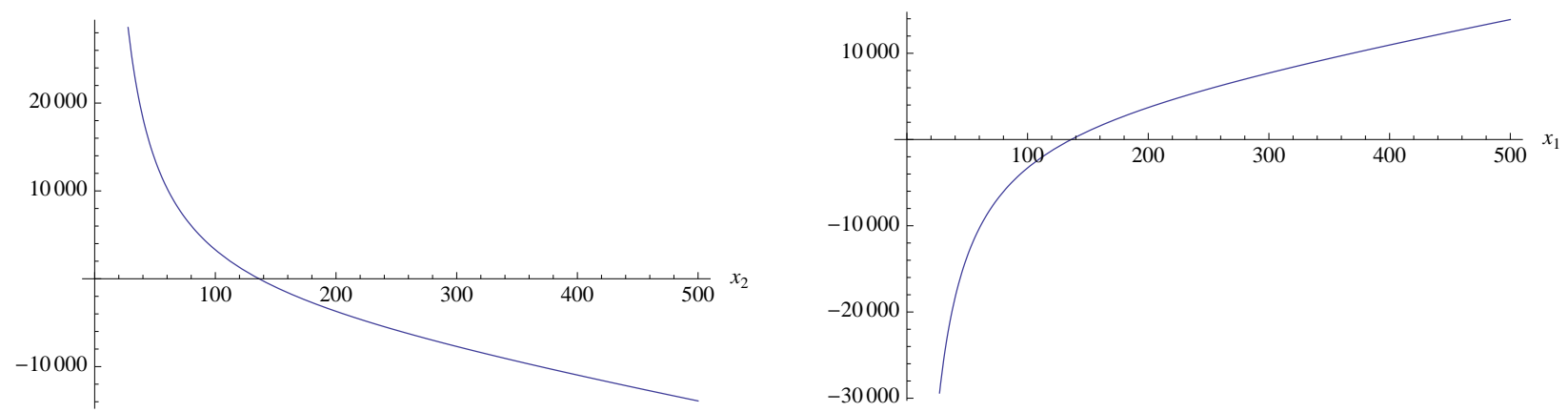

Figure 7: Left-hand sides of constraints (15) (left) and (16) (right) after interchanging the values of $B_{1}, B_{2}$ in Table 2 
each constraint is satisfied on a convex subset of the real line, which makes the feasible region of the surrogate problem connected and hence convex. Note that strict concavity of one constraint may still persist (which is the case when $B_{1} \neq B_{2}$, cf. (17)). Nevertheless, both left-hand sides prove to be quasiconvex functions (see Appendix $\mathrm{J}$ for a definition); given the strict convexity of objective function (14), this guarantees optimality of a KKT solution (see Proposition J.2 in Appendix J).

Unfortunately, weak Pareto-efficiency of the buyer's possible cost structures does not lead to quasiconvexity of constraints in the original problem (1)-(6), so that the above argument does not carry over to problem (1)-(6). Despite that, there still holds the following

Proposition 4. Suppose that the buyer's possible cost structures are both weakly Pareto-efficient. Then a KKT solution of problem (1)-(6) is an optimal solution. Specifically, the following is true:

i) a set of offers of type 1 to 5 satisfying the respective feasibility conditions and necessary optimality conditions is optimal;

ii) a set of offers of type 6 satisfying the respective feasibility conditions and necessary optimality conditions cannot exist, unless it coincides with a set of offers from i);

iii) sets of offers of types 7 and 8 are undefined.

Proof. See Appendix J.

Proposition 4 thus establishes that weak Pareto-efficiency of the buyer's cost structures guarantees optimality of a KKT solution of the original problem, too. To obtain this result, we employ the Generalized Lagrange Multiplier approach (see Appendix J for details) and prove that under this condition, the restricted Lagrangian function of the problem turns out to be convex, what guarantees optimality of the solution in question - despite the fact that the problem remains nonconvex. This can be explained as follows. As we know from Section 3, weak Pareto-efficiency of the buyer's possible cost structures implies the single-crossing property. Under single-crossing, the functions in Figure 7 happen to be monotonic. This, in turn, leads to convexity of the restricted Lagrangian function and thus ensures optimality of the respective stationary point (see the proof of Proposition 4 in Appendix J for details).

An immediate consequence of the proof of Proposition 4 is the following

Corollary 3. The set of offers of type 1 is optimal whenever it satisfies the respective feasibility conditions. The buyer's possible cost structures do not need to be both weakly Pareto-efficient.

Indeed, as it is optimal for the supplier to offer $\left(x_{i}, z_{i}\right)$ with $x_{i}=\operatorname{JELS}_{i}$ and $z_{i}=K_{i}^{A}\left(x_{i}\right)-$ $K_{i}^{A}\left(x_{A, i}^{*}\right)$ to the buyer with the cost structure no. $i$ (Banerjee, 1986; Sucky, 2006), the set of offers 
of type 1 is an optimal solution to a relaxed problem (1)-(3), (6). If it happens to be feasible to the original problem (1)-(6) as well, then it obviously represents its optimal solution, irrespective of the relation between the buyer's possible cost structures (see also Lemma I.1 in Appendix I).

\section{Conclusion}

In the present paper we have conducted an optimality analysis of the single supplier-single buyer bargaining model with asymmetric information introduced by Sucky (2004, 2006), by taking his original analysis as our starting point. Our contribution to the knowledge about this problem and its solution is four-fold. First, we proposed a refinement of the original closed-form solution of the problem, thus simplifying problem solving and its analysis. Second, we have proven that KKT conditions are necessary for optimality, and demonstrated that the problem on hand may, in general, possess non-optimal stationary points due to non-convexity. Third, we have proven that certain types of stationary points happen to be always dominated, which eases the analytical solution of the problem by allowing to restrict attention only to specific types of stationary points. Fourth, we derived an easily perceivable optimality condition that requires a weak Pareto-efficiency of the buyer's possible cost structures, under which any stationary point of the problem proves to be its optimal solution.

We thus contribute to both analytical and numerical approaches to solving the problem. It must be noted that in general, the analytical approach still requires to check potentially multiple candidate solutions against the respective feasibility and necessary optimality conditions, in order to determine the stationary points of the problem, and further evaluate the objective function at each stationary point. The first, third and fourth of the above results nevertheless reduce the required effort and simplify any subsequent parametric analysis of the problem substantially. The fourth result ensures, in addition, a successful solution of the problem by means of conventional numerical techniques, e.g. with a general-purpose solver, as it ascertains the user that the solver converging to a KKT solution converges to the true optimum of the problem.

The properties of the optimal sets of offers that we have derived are, on the one hand, in line with other existing results on discrete-type adverse selection problems: there is no distortion from the first-best for at least one buyer type, and there exist five generic types of optimal solutions - even if the single-crossing condition does not hold. However, in the latter case, there exists a possibility of bunching, which is in line with the results obtained in continuous-type settings (Araujo and Moreira, 2010; Rochet and Choné, 1998). We have fully characterized the conditions for emergence of bunching in our setting.

An interesting direction for the future research would be to extend the above results to more general settings - e.g. involving product quality aspects (Affisco et al., 2002; Liu and Çetinkaya, 
2007) and more general production and shipment policies (Ben-Daya et al., 2008), more than two or a continuum of the buyer's possible cost structures (McAfee and McMillan, 1988; Rochet and Stole, 2003), and more general distributions of bargaining power between supply chain members.

\section{Acknowledgement}

The authors are thankful to Professor Eric Sucky and an anonymous reviewer for their insightful comments and suggestions on the manuscript. The first author gratefully acknowledges the financial support by the Leverhulme Trust, UK during his research stay at the Manchester Metropolitan University Business School in 2010 and the financial support by the European University Viadrina Frankfurt (Oder), Germany, where this research has been partly conducted.

\section{References}

Affisco, J. F., Paknejad, M. J., Nasri, F., 2002. Quality improvement and setup reduction in the joint economic lot size model. European Journal of Operational Research 142 (3), 497-508.

Araujo, A., Moreira, H., 2010. Adverse selection problems without the Spence-Mirrlees condition. Journal of Economic Theory 145 (3), 1113-1141.

Banerjee, A., 1986. A joint economic-lot-size model for purchaser and vendor. Decision Sciences 17 (3), $292-311$.

Bazaraa, M. S., Sherali, H. D., Shetty, C. M., 2006. Nonlinear Programming: Theory and Algorithms, 3rd Edition. Wiley, Hoboken, NJ.

Ben-Daya, M., Darwish, M., Ertogral, K., 2008. The joint economic lot sizing problem: Review and extensions. European Journal of Operational Research 185 (2), 726-742.

Brooks, R., Geoffrion, A., 1966. Finding Everett's Lagrange multipliers by linear programming. Operations Research $14(6), 1149-1153$.

Burnetas, A., Gilbert, S. M., Smith, C. E., 2007. Quantity discounts in single-period supply contracts with asymmetric demand information. IIE Transactions 39 (5), 465-479.

Cachon, G. P., Zhang, F., 2006. Procuring fast delivery: Sole sourcing with information asymmetry. Management Science 52 (6), 881-896.

Cecchini, M., Ecker, J., Kupferschmid, M., Leitch, R., 2013. Solving nonlinear principal-agent problems using bilevel programming. European Journal of Operational Research 230 (2), 364-373.

Corbett, C. J., de Groote, X., 2000. A supplier's optimal quantity discount policy under asymmetric information. Management Science 46 (3), 444-450.

Everett, H., 1963. Generalized Lagrange multiplier method for solving problems of optimum allocation of resources. Operations Research 11 (3), 399-417.

Figalli, A., Kim, Y.-H., McCann, R. J., 2011. When is multidimensional screening a convex program? Journal of Economic Theory 146 (2), 454-478.

Glock, C. H., 2012. The joint economic lot size problem: A review. International Journal of Production Economics $135(2), 671-686$.

Ha, A. Y., 2001. Supplier-buyer contracting: Asymmetric cost information and cutoff level policy for buyer participation. Naval Research Logistics 48 (1), 41-64. 
Hellwig, M. F., 2010. Incentive problems with unidimensional hidden characteristics: A unified approach. Econometrica 78 (4), 1201-1237.

Hestenes, M. R., 1975. Optimization theory. Wiley, New York.

Horsley, A., Wrobel, A. J., Sep. 2004. First and second-order conditions in constrained optimisation. CDAM Research Report LSE-CDAM-2003-15, London School of Economics, United Kingdom.

Jullien, B., 2000. Participation constraints in adverse selection models. Journal of Economic Theory 93 (1), 1-47.

Kerschbamer, R., Maderner, N., 1998. Are two a good representative for many? Journal of Economic Theory 83 (1), 90-104.

Kreps, D. M., 1990. A course in microeconomic theory. Harvester Wheatsheaf, New York.

Laffont, J.-J., Martimort, D., 2002. The Theory of Incentives: The Principal-Agent Model. Princeton University Press, Princeton.

Lau, A. H. L., Lau, H.-S., Zhou, Y.-W., 2006. Considering asymmetrical manufacturing cost information in a twoechelon system that uses price-only contracts. IIE Transactions 38 (3), 253-271.

Liu, X., Çetinkaya, S., 2007. A note on "quality improvement and setup reduction in the joint economic lot size model". European Journal of Operational Research 182 (1), 194-204.

Liu, X., Cetinkaya, S., 2009. Designing supply contracts in supplier vs buyer-driven channels: The impact of leadership, contract flexibility and information asymmetry. IIE Transactions 41 (8), 687-701.

Lutze, H., Özer, Ö., 2008. Promised lead-time contracts under asymmetric information. Operations Research 56 (4), 898-915.

Mangasarian, O. L., 1965. Pseudo-convex functions. Journal of the Society for Industrial and Applied Mathematics, Series A: Control 3 (2), 281-290.

Mangasarian, O. L., 1969. Nonlinear Programming. McGraw-Hill, New York.

Mangasarian, O. L., Fromovitz, S., 1967. The Fritz John necessary optimality conditions in the presence of equality and inequality constraints. Journal of Mathematical Analysis and Applications 17, 37-47.

Maskin, E., Riley, J., 1984. Monopoly with incomplete information. RAND Journal of Economics 15 (2), $171-196$.

McAfee, R. P., McMillan, J., 1988. Multidimensional incentive compatibility and mechanism design. Journal of Economic Theory 46 (2), 335-354.

Morrow, W. R., Jun. 2011. Hessian-free methods for checking the second-order sufficient conditions in equalityconstrained optimization and equilibrium problems. Working Paper arXiv:1106.0898v1, arXiv.org.

Myerson, R. B., 1979. Incentive compatibility and the bargaining problem. Econometrica 47 (1), 61-73.

Myerson, R. B., 1982. Optimal coordination mechanisms in generalized principal-agent problems. Journal of Mathematical Economics 10 (1), 67-81.

Pibernik, R., Zhang, Y., Kerschbaum, F., Schröpfer, A., 2011. Secure collaborative supply chain planning and inverse optimization - The JELS model. European Journal of Operational Research 208 (1), 75-85.

Rochet, J.-C., Choné, P., 1998. Ironing, sweeping, and multidimensional screening. Econometrica 66 (4), $783-826$.

Rochet, J.-C., Stole, L. A., 2003. The economics of multidimensional screening. In: Dewatripont, M., Hansen, L. P., Turnovsky, S. J. (Eds.), Advances in Economics and Econometrics. Theory and Applications, Eighth World Congress, Volume I. Cambridge University Press, Cambridge, Ch. 5, pp. 150-197.

Schottmüller, C., 2015. Adverse selection without single crossing: Monotone solutions. Journal of Economic Theory 158 (Part A), 127-164.

Solodov, M. V., 2011. Constraint qualifications. In: Cochran, J. J., Cox, Jr., L. A., Keskinocak, P., Kharoufeh, J. P., Smith, J. C. (Eds.), Wiley Encyclopedia of Operations Research and Management Science. Wiley, Hoboken, NJ. Sucky, E., 2004. Coordinated order and production policies in supply chains. OR Spectrum 26 (4), 493-520. 
Sucky, E., 2006. A bargaining model with asymmetric information for a single supplier-single buyer problem. European Journal of Operational Research 171 (2), 516-535.

van Ackere, A., 1993. The principal/agent paradigm: Its relevance to various functional fields. European Journal of Operational Research 70 (1), 83-103.

Voigt, G., Inderfurth, K., 2011. Supply chain coordination and setup cost reduction in case of asymmetric information.

OR Spectrum 33 (1), 99-122.

Wang, J.-C., Lau, H.-S., Lau, A. H. L., 2009. When should a manufacturer share truthful manufacturing cost information with a dominant retailer? European Journal of Operational Research 197 (1), 266-286.

Yang, Z., Aydin, G., Babich, V., Beil, D. R., 2009. Quantity discounts in single-period supply contracts with asymmetric demand information. Management Science 55 (2), 192-209.

\section{Appendix A. Karush-Kuhn-Tucker conditions}

Karush-Kuhn-Tucker (KKT) conditions and problem constraints (2)-(5) comprise the following system of equations and inequalities (Bazaraa et al., 2006; Sucky, 2006):

$$
\begin{array}{lll}
\omega_{1} \cdot \frac{d K^{P}\left(x_{1}\right)}{d x_{1}}+\lambda_{1} \cdot \frac{d K_{1}^{A}\left(x_{1}\right)}{d x_{1}}+\mu_{1} \cdot \frac{d K_{1}^{A}\left(x_{1}\right)}{d x_{1}}-\mu_{2} \cdot \frac{d K_{2}^{A}\left(x_{1}\right)}{d x_{1}}=0 \\
\omega_{2} \cdot \frac{d K^{P}\left(x_{2}\right)}{d x_{2}}+\lambda_{2} \cdot \frac{d K_{2}^{A}\left(x_{2}\right)}{d x_{2}}-\mu_{1} \cdot \frac{d K_{1}^{A}\left(x_{2}\right)}{d x_{2}}+\mu_{2} \cdot \frac{d K_{2}^{A}\left(x_{2}\right)}{d x_{2}}=0 \\
\omega_{1}-\lambda_{1}-\mu_{1}+\mu_{2}=0 & \\
\omega_{2}-\lambda_{2}+\mu_{1}-\mu_{2}=0 & \text { and } & \lambda_{1} \cdot\left(K_{1}^{A}\left(x_{A, 1}^{*}\right)-K_{1}^{A}\left(x_{1}\right)+z_{1}\right)=0 \\
K_{1}^{A}\left(x_{A, 1}^{*}\right)-K_{1}^{A}\left(x_{1}\right)+z_{1} \geq 0 & \text { and } & \lambda_{2} \cdot\left(K_{2}^{A}\left(x_{A, 2}^{*}\right)-K_{2}^{A}\left(x_{2}\right)+z_{2}\right)=0 \\
K_{2}^{A}\left(x_{A, 2}^{*}\right)-K_{2}^{A}\left(x_{2}\right)+z_{2} \geq 0 & \text { and } & \mu_{1} \cdot\left(K_{1}^{A}\left(x_{2}\right)-z_{2}-K_{1}^{A}\left(x_{1}\right)+z_{1}\right)=0 \\
K_{1}^{A}\left(x_{2}\right)-z_{2}-K_{1}^{A}\left(x_{1}\right)+z_{1} \geq 0 & \text { and } & \mu_{2} \cdot\left(K_{2}^{A}\left(x_{1}\right)-z_{1}-K_{2}^{A}\left(x_{2}\right)+z_{2}\right)=0 \\
K_{2}^{A}\left(x_{1}\right)-z_{1}-K_{2}^{A}\left(x_{2}\right)+z_{2} \geq 0 & \text { and }
\end{array}
$$

A set of offers that satisfies (A.1)-(A.8) for some nonnegative values of Lagrange multipliers $\lambda_{1}, \lambda_{2}, \mu_{1}, \mu_{2}$ is called a $K K T$ solution of problem (1)-(6), or its stationary point. 


\section{Appendix B. Refining KKT solutions of types 4 to 6}

\section{B.1. KKT solutions of type 4}

KKT solutions of type 4 assume the case $\lambda_{1}>0, \lambda_{2}>0, \mu_{1}>0, \mu_{2}=0$ (Sucky, 2006, p. 531). By solving KKT conditions (A.1)-(A.8) under this assumption, one obtains the expressions of two possible sets of offers with the following order sizes and side payments (Sucky, 2006, pp. 527-528):

$$
\begin{aligned}
x_{1} & =\operatorname{JELS}_{1} \\
x_{1} & =K_{1}^{A}\left(x_{1}\right)-K_{1}^{A}\left(x_{A, 1}^{*}\right) \\
x_{2_{1,2}} & =-\frac{K_{2}^{A}\left(x_{A, 2}^{*}\right)-K_{1}^{A}\left(x_{A, 1}^{*}\right)}{h_{A, 1}-h_{A, 2}} \pm \sqrt{\left(\frac{K_{2}^{A}\left(x_{A, 2}^{*}\right)-K_{1}^{A}\left(x_{A, 1}^{*}\right)}{h_{A, 1}-h_{A, 2}}\right)^{2}-\frac{2 \cdot\left(B_{1}-B_{2}\right) \cdot d}{h_{A, 1}-h_{A, 2}}} \\
z_{2} & =K_{2}^{A}\left(x_{2}\right)-K_{2}^{A}\left(x_{A, 2}^{*}\right)
\end{aligned}
$$

We provide their detailed derivation in Section D.1 of Appendix D. Further, we show that the expressions of $x_{2_{1,2}}$ in (B.2) can be considerably simplified, so that (B.2) takes the following form:

$$
x_{2_{1,2}}=\sqrt{2 d} \cdot \frac{\sqrt{B_{1}} \pm \sqrt{B_{2}}}{\sqrt{h_{A, 1}} \pm \sqrt{h_{A, 2}}}
$$

where the same sign (either plus or minus) simultaneously applies in the numerator and the denominator. We refer the reader to Section D.2 of Appendix D for a detailed proof. Note that the order size $x_{2_{2}}$ is positive only if the buyer's possible cost structures $\left\{B_{1}, h_{A, 1}\right\}$ and $\left\{B_{2}, h_{A, 2}\right\}$ strictly dominate one another in Pareto sense.

Furthermore, we revise the necessary optimality condition for a set of offers of type 4 and obtain it in the following form (see Section D.1 of Appendix D for a detailed derivation):

$$
\left\{\begin{array}{l}
x_{2}=\mathrm{JELS}_{2}, \quad \text { if } x_{A, 1}^{*}=x_{A, 2}^{*} \\
0 \leq \mu_{1} \leq \omega_{1}, \quad \text { otherwise }
\end{array}\right.
$$

where

$$
\mu_{1}=\frac{\left(\frac{d}{p} h_{P}+h_{A, 2}\right) x_{2}^{2}-2 d\left(R+B_{2}\right)}{\left(h_{A, 1}-h_{A, 2}\right) x_{2}^{2}-2 d\left(B_{1}-B_{2}\right)} \cdot \omega_{2} .
$$

Condition (B.5) differs from the original necessary optimality condition $\mu_{1}>0$ (Sucky, 2006) in three ways: (i) it imposes an upper bound on $\mu_{1}$; (ii) it relaxes the strict inequality to the nonstrict one in order to accommodate degenerate solutions; (iii) it accounts for the possibility of a zero denominator in (B.6). Table 1 displays condition (B.5) by separating it into two rows. 


\section{B.2. KKT solutions of type 5}

KKT solutions of type 5 are associated with the case $\lambda_{1}>0, \lambda_{2}>0, \mu_{1}=0, \mu_{2}>0$ and are given by equations (34)-(35), (A.25), (A.34) in Sucky (2006). They are symmetrical to the respective KKT solutions of type 4 and can be obtained from the latter by exchanging the indices of the buyer's possible cost structures. Following equation (B.4), we can therefore considerably simplify the original expressions of the order size $x_{1}$ in a set of offers of type 5 as follows:

$$
x_{1_{1,2}}=\sqrt{2 d} \cdot \frac{\sqrt{B_{1}} \pm \sqrt{B_{2}}}{\sqrt{h_{A, 1}} \pm \sqrt{h_{A, 2}}}
$$

where the same sign (either plus or minus) simultaneously applies in both the numerator and the denominator. Similarly, we adjust the original necessary optimality condition (A.34) in Sucky (2006) to:

$$
\begin{cases}x_{1}=\mathrm{JELS}_{1}, & \text { if } x_{A, 1}^{*}=x_{A, 2}^{*} \\ 0 \leq \mu_{2} \leq \omega_{2}, & \text { otherwise }\end{cases}
$$

where

$$
\mu_{2}=\frac{\left(\frac{d}{p} h_{P}+h_{A, 1}\right) x_{1}^{2}-2 d\left(R+B_{1}\right)}{\left(h_{A, 2}-h_{A, 1}\right) x_{1}^{2}-2 d\left(B_{2}-B_{1}\right)} \cdot \omega_{1} .
$$

\section{B.3. KKT solutions of type 6}

KKT solutions of type 6 are associated with the case $\lambda_{1}, \lambda_{2}, \mu_{1}, \mu_{2}>0$ and given by equations (36)-(37), (A.33), (A.34) in Sucky (2006). We can immediately see that the original expressions of the order sizes $x_{1}, x_{2}$ are considerably simplified by the above equations (B.4) and (B.7).

Further, the necessary optimality condition has to be extended by a similar approach as in Section B.1 to:

$$
\begin{cases}\left(x_{1}=\mathrm{JELS}_{1}\right) \wedge\left(x_{2}=\mathrm{JELS}_{2}\right), & \text { if } x_{A, 1}^{*}=x_{A, 2}^{*} \\ \mu_{1}, \mu_{2} \geq 0, \quad-\omega_{2} \leq \mu_{1}-\mu_{2} \leq \omega_{1}, & \text { otherwise }\end{cases}
$$

where $\mu_{1}, \mu_{2}$ are as defined in equations (B.6) and (B.9) above. We provide specific details in Appendix E. 


\section{Appendix C. Deriving KKT solutions of type 7 and 8}

As indicated in Section 3, we contribute to the original results of Sucky (2006) by deriving two additional types of KKT solutions of problem (1)-(6). They correspond to cases no. 5 and 6 in Sucky (2006, Table 5); to maintain the original numbering of KKT solutions, we designate the new solution types as type 7 and 8 , respectively, and derive them below in the closed form. To simplify the subsequent exposition, let

$$
\mathcal{A}=2 d \cdot \frac{B_{1}-B_{2}}{h_{A, 1}-h_{A, 2}}
$$

\section{C.1. KKT solutions of type 7}

KKT solutions of type 7 correspond to case no. 5 as per Table 5 in Sucky (2006) — being described by the following combination of Lagrange multipliers: $\lambda_{1}=0$ and $\lambda_{2}, \mu_{1}, \mu_{2}>0$. Solving KKT conditions (A.1)-(A.8), as shown in Appendix F, yields two possible sets of offers. The first one is characterised by the order sizes

$$
x_{1_{1}}=x_{2_{1}}=\sqrt{\mathcal{A}}
$$

and the following necessary optimality condition:

$$
x_{1_{1}}=x_{2_{1}}=\mathrm{JELS}_{2}
$$

where $\mathcal{A}$ is as defined by (C.1). The second set of offers is characterised by the order sizes

$$
\begin{aligned}
& x_{1_{2}}=\sqrt{2 d \cdot \frac{\omega_{1} \cdot\left(R+B_{2}\right)+\omega_{2} \cdot\left(\frac{d}{p} h_{P}+h_{A, 2}\right) \cdot\left(B_{1}-B_{2}\right) /\left(h_{A, 1}-h_{A, 2}\right)}{\omega_{1} \cdot\left(\frac{d}{p} h_{P}+h_{A, 2}\right)+\omega_{2} \cdot\left(R+B_{2}\right) \cdot\left(h_{A, 1}-h_{A, 2}\right) /\left(B_{1}-B_{2}\right)}} \\
& x_{2_{2}}=\mathcal{A} / x_{1_{2}}
\end{aligned}
$$

and the following necessary optimality condition:

$$
\frac{\left(\frac{d}{p} h_{P}+h_{A, 1}\right) x_{2_{2}}^{2}-2 d\left(R+B_{1}\right)}{\left(h_{A, 1}-h_{A, 2}\right) x_{2_{2}}^{2}-2 d\left(B_{1}-B_{2}\right)} \cdot \omega_{2} \geq 1 .
$$

The side payments $z_{1}, z_{2}$ to be offered in conjunction with the respective order sizes $x_{1}, x_{2}$ are determined in either case as follows:

$$
z_{1}=K_{2}^{A}\left(x_{1}\right)-K_{2}^{A}\left(x_{A, 2}^{*}\right) \quad z_{2}=K_{2}^{A}\left(x_{2}\right)-K_{2}^{A}\left(x_{A, 2}^{*}\right)
$$




\begin{tabular}{lll}
\hline Supplier & Buyer: cost structure no. 1 & cost structure no. 2 \\
\hline $\begin{array}{l}R=100, h_{P}=10 \\
p=15000\end{array}$ & $B_{1}=100, h_{A, 1}=30, \omega_{1}=0.7$ & $B_{2}=40, h_{A, 2}=20, \omega_{2}=0.3$ \\
& $d=5000$ & \\
\hline & $\Rightarrow x_{A, 1}^{*} \approx 182.6$ & $\Rightarrow x_{A, 2}^{*} \approx 141.4$ \\
& $\Rightarrow K_{1}^{A}\left(x_{A, 1}^{*}\right) \approx 5477.2$ & $\Rightarrow K_{2}^{A}\left(x_{A, 2}^{*}\right) \approx 2828.4$ \\
\hline Set of offers of type 7: & $\left(x_{1}, z_{1}\right) \approx(244.95,437.56)$ & $\left(x_{2}, z_{2}\right) \approx(244.95,437.56)$ \\
\hline
\end{tabular}

Table C.3: Example of an optimal set of offers of type 7

and have to satisfy the following feasibility condition:

$$
K_{1}^{A}\left(x_{2}\right)-z_{2} \leq K_{1}^{A}\left(x_{A, 1}^{*}\right)
$$

We refer the reader to Appendix F for full details of deriving the above results. Note that sets of offers of type 7 are defined only when $\mathcal{A}>0$ holds — what in turn requires that the buyer's possible cost structures strictly dominate one another in Pareto sense. The analysis in Appendix F further provides us with the following two observations.

Observation 1. If a set of offers of type 7 with $x_{1}=x_{2}$ satisfies the respective necessary optimality condition then there must necessarily hold $\mathrm{JELS}_{1}=\mathrm{JELS}_{2}$.

Observation 2. If a set of offers of type 7 with $x_{1} \neq x_{2}$ satisfies the respective necessary optimality condition then there must necessarily hold $\omega_{1} \neq \omega_{2}$.

Table C.3 provides an example in which a set offers of type 7 happens to be a KKT solution of problem (1)-(6) and its optimal solution, too. Furthermore, this set of offers yields supply-chain coordination because it leads either buyer type to adopting the system-optimal joint economic lot size, as Observation 1 and condition (C.3) indicate. Note that the buyer's choice from this set of offers will reveal no information to the supplier because both offers coincide.

\section{C.2. KKT solutions of type 8}

These KKT solutions correspond to case no. 6 as per Table 5 in Sucky (2006), being described by the following combination of Lagrange multipliers: $\lambda_{1}>0, \lambda_{2}=0$ and $\mu_{1}, \mu_{2}>0$. This case is symmetric to the one considered in Section C.1 above, therefore KKT solutions of type 8 can be obtained by exchanging the indices of the buyer's assumed cost structures in equations (C.2)-(C.8). We collate the results in Table 1.

Note that sets of offers of type 8 are undefined for problem instances with $\mathcal{A} \leq 0$. Furthermore, Observations 1 and 2 made in Section C.1 above equally apply to sets of offers of type 8 . 


\section{Appendix D. Detailed derivation of KKT solutions of type 4}

\section{D.1. Derivation procedure}

KKT solutions of type 4 assume the case $\lambda_{1}>0, \lambda_{2}>0, \mu_{1}>0, \mu_{2}=0$ (Sucky, 2006, p. 531). Refer to KKT conditions (A.1)-(A.8). From (A.3) we immediately have $\lambda_{1}+\mu_{1}=\omega_{1}$, what allows us to rewrite condition (A.1) as follows:

$$
\omega_{1} \cdot\left(\frac{d K^{P}\left(x_{1}\right)}{d x_{1}}+\frac{d K_{1}^{A}\left(x_{1}\right)}{d x_{1}}\right)=0
$$

By definition of the functions involved, the above equation yields the following solution:

$$
x_{1}=\mathrm{JELS}_{1}
$$

Since $\lambda_{1}, \lambda_{2} \neq 0$ holds by assumption, we immediately have from (A.5) and (A.6):

$$
z_{i}=K_{i}^{A}\left(x_{i}\right)-K_{i}^{A}\left(x_{A, i}^{*}\right) \quad(i=1,2)
$$

Substituting the expressions of $z_{1}, z_{2}$ from (D.2) into the second part of (A.7) and taking into account that $\mu_{1} \neq 0$ by assumption, we obtain the following equation:

$$
K_{1}^{A}\left(x_{2}\right)-K_{2}^{A}\left(x_{2}\right)=K_{1}^{A}\left(x_{A, 1}^{*}\right)-K_{2}^{A}\left(x_{A, 2}^{*}\right) .
$$

By definition of the functions involved, this equation has two possible roots (Sucky, 2006):

$$
x_{2_{1,2}}=\frac{K_{1}^{A}\left(x_{A, 1}^{*}\right)-K_{2}^{A}\left(x_{A, 2}^{*}\right)}{h_{A, 1}-h_{A, 2}} \pm \sqrt{\left(\frac{K_{1}^{A}\left(x_{A, 1}^{*}\right)-K_{2}^{A}\left(x_{A, 2}^{*}\right)}{h_{A, 1}-h_{A, 2}}\right)^{2}-2 d \cdot \frac{B_{1}-B_{2}}{h_{A, 1}-h_{A, 2}}}
$$

The above formula can be substantially simplified as shown in Section D.2 below, which yields:

$$
x_{2_{1,2}}=\sqrt{2 d} \cdot \frac{\sqrt{B_{1}} \pm \sqrt{B_{2}}}{\sqrt{h_{A, 1}} \pm \sqrt{h_{A, 2}}}
$$

where the same sign — either plus or minus - simultaneously applies in the numerator and the denominator.

Further, given that $\mu_{2}=0$ holds by assumption, condition (A.8) holds if and only if the feasibility constraint it contains is satisfied. Taking into account (D.2) for $i=2$, the said constraint can be rewritten as the following feasibility condition:

$$
K_{2}^{A}\left(x_{1}\right)-z_{1} \geq K_{2}^{A}\left(x_{A, 2}^{*}\right)
$$


We now turn to KKT condition (A.2). From (A.4) we immediately have $\lambda_{2}=\mu_{1}+\omega_{2}$. This allows us to rewrite (A.2) in the following form:

$$
\omega_{2} \cdot\left(\frac{d K^{P}\left(x_{2}\right)}{d x_{2}}+\frac{d K_{2}^{A}\left(x_{2}\right)}{d x_{2}}\right)+\mu_{1} \cdot\left(\frac{d K_{2}^{A}\left(x_{2}\right)}{d x_{2}}-\frac{d K_{1}^{A}\left(x_{2}\right)}{d x_{2}}\right)=0 .
$$

Assume that the second parenthetical expression in the above equation is non-zero. By taking into account the definitions of the functions involved, we then obtain:

$$
\mu_{1}=\frac{\frac{d K^{P}\left(x_{2}\right)}{d x_{2}}+\frac{d K_{2}^{A}\left(x_{2}\right)}{d x_{2}}}{\frac{d K_{1}^{A}\left(x_{2}\right)}{d x_{2}}-\frac{d K_{2}^{A}\left(x_{2}\right)}{d x_{2}}} \cdot \omega_{2}=\frac{\left(\frac{d}{p} h_{P}+h_{A, 2}\right) x_{2}^{2}-2 d\left(R+B_{2}\right)}{\left(h_{A, 1}-h_{A, 2}\right) x_{2}^{2}-2 d\left(B_{1}-B_{2}\right)} \cdot \omega_{2}
$$

For a KKT solution, we have to require that $\mu_{1} \geq 0$. Furthermore, from (A.3) we have $\lambda_{1}=\omega_{1}-\mu_{1}$ and have to require $\lambda_{1} \geq 0$ as well. Both these requirements yield the following necessary optimality condition:

$$
0 \leq \mu_{1} \leq \omega_{1}
$$

Note that having $\mu_{1}=0$ or $\mu_{1}=\omega_{1}$ designates a degenerate KKT solution. It is easy to verify that in the former case, a set of offers of type 4 coincides with that of type 1, whereas in the latter case - with that of type 3. Despite the assumption $\lambda_{1}, \mu_{1}>0$ adopted for deriving KKT solutions of type 4, we thus still permit either Lagrange multiplier to take on the zero value, in order to consistently classify the coinciding sets of offers as a KKT solution using the feasibility and necessary optimality conditions of either solution type.

Assume now that the second parenthetical expression in (D.5) equals zero. Then equation (D.5) holds if and only if its first parenthetical expression equals zero as well; in such a case we can set $\mu_{1}$ to any value within the bounds $0 \leq \mu_{1} \leq \omega_{1}$. It is straightforward to verify that the first parenthetical expression in (D.5) equals zero if and only if

$$
x_{2}=\mathrm{JELS}_{2}
$$

Note that solution (D.1)-(D.3) coincides then with the set of offers of type 1, see Table 1 (thus it makes sense to deliberately set $\mu_{1}=0$ because this is assumed for the set of offers of type 1 , see Sucky, 2006, p. 531). In Section D.3 below we show that an order size $x_{2}>0$ defined by (D.3) turns the second parenthetical expression in (D.5) to zero if and only if

$$
\frac{B_{1}}{B_{2}}=\frac{h_{A, 1}}{h_{A, 2}}
$$


Note that this is equivalent to having $x_{A, 1}^{*}=x_{A, 2}^{*}$ - i.e., equal economic order quantities associated with the buyer's possible cost structures. We thus extend the necessary optimality condition (D.7) to the following one:

$$
\begin{cases}x_{2}=\mathrm{JELS}_{2}, & \text { if } x_{A, 1}^{*}=x_{A, 2}^{*}, \\ (\mathrm{D} .7), & \text { otherwise. }\end{cases}
$$

\section{D.2. Simplifying the expressions of the order size $x_{2}$}

We obtain the required expressions of the order size $x_{2}$ in a set of offers of type 4 by performing the following transformations of the original expressions (B.2):

$$
\begin{aligned}
& x_{2_{1,2}}=-\frac{K_{2}^{A}\left(x_{A, 2}^{*}\right)-K_{1}^{A}\left(x_{A, 1}^{*}\right)}{h_{A, 1}-h_{A, 2}} \pm \sqrt{\left(\frac{K_{2}^{A}\left(x_{A, 2}^{*}\right)-K_{1}^{A}\left(x_{A, 1}^{*}\right)}{h_{A, 1}-h_{A, 2}}\right)^{2}-\frac{2 \cdot\left(B_{1}-B_{2}\right) \cdot d}{h_{A, 1}-h_{A, 2}}} \\
& =-\frac{\sqrt{2 d B_{2} h_{A, 2}}-\sqrt{2 d B_{1} h_{A, 1}}}{h_{A, 1}-h_{A, 2}} \pm \sqrt{\frac{\left(\sqrt{2 d B_{2} h_{A, 2}}-\sqrt{2 d B_{1} h_{A, 1}}\right)^{2}}{\left(h_{A, 1}-h_{A, 2}\right)^{2}}-\frac{2 \cdot\left(B_{1}-B_{2}\right) \cdot d}{h_{A, 1}-h_{A, 2}}} \\
& =\sqrt{2 d} \cdot\left[\frac{\sqrt{B_{1} h_{A, 1}}-\sqrt{B_{2} h_{A, 2}}}{h_{A, 1}-h_{A, 2}} \pm \sqrt{\frac{\left(\sqrt{B_{1} h_{A, 1}}-\sqrt{B_{2} h_{A, 2}}\right)^{2}}{\left(h_{A, 1}-h_{A, 2}\right)^{2}}-\frac{B_{1}-B_{2}}{h_{A, 1}-h_{A, 2}}}\right] \\
& =\sqrt{2 d} \cdot\left[\frac{\sqrt{B_{1} h_{A, 1}}-\sqrt{B_{2} h_{A, 2}}}{h_{A, 1}-h_{A, 2}} \pm\right. \\
& \left. \pm \sqrt{\frac{B_{1} h_{A, 1}-2 \sqrt{B_{1} B_{2} h_{A, 1} h_{A, 2}}+B_{2} h_{A, 2}-B_{1} h_{A, 1}+B_{1} h_{A, 2}+B_{2} h_{A, 1}-B_{2} h_{A, 2}}{\left(h_{A, 1}-h_{A, 2}\right)^{2}}}\right] \\
& =\sqrt{2 d} \cdot\left[\frac{\sqrt{B_{1} h_{A, 1}}-\sqrt{B_{2} h_{A, 2}}}{h_{A, 1}-h_{A, 2}} \pm \sqrt{\frac{B_{1} h_{A, 2}-2 \sqrt{B_{1} B_{2} h_{A, 1} h_{A, 2}}+B_{2} h_{A, 1}}{\left(h_{A, 1}-h_{A, 2}\right)^{2}}}\right] \\
& =\sqrt{2 d} \cdot\left[\frac{\sqrt{B_{1} h_{A, 1}}-\sqrt{B_{2} h_{A, 2}}}{h_{A, 1}-h_{A, 2}} \pm \sqrt{\frac{\left(\sqrt{B_{1} h_{A, 2}}-\sqrt{B_{2} h_{A, 1}}\right)^{2}}{\left(h_{A, 1}-h_{A, 2}\right)^{2}}}\right] .
\end{aligned}
$$

The latter expression can, without loss of generality, be further simplified to

$$
\begin{aligned}
& \sqrt{2 d} \cdot\left[\frac{\sqrt{B_{1} h_{A, 1}}-\sqrt{B_{2} h_{A, 2}}}{h_{A, 1}-h_{A, 2}} \pm \frac{\sqrt{B_{1} h_{A, 2}}-\sqrt{B_{2} h_{A, 1}}}{h_{A, 1}-h_{A, 2}}\right] \\
= & \sqrt{2 d} \cdot \frac{\sqrt{B_{1}}\left(\sqrt{h_{A, 1}} \pm \sqrt{h_{A, 2}}-\sqrt{B_{2}}\left(\sqrt{h_{A, 2}} \pm \sqrt{h_{A, 1}}\right)\right.}{h_{A, 1}-h_{A, 2}}
\end{aligned}
$$


what, without loss of generality, gives

$$
x_{2_{1,2}}=\sqrt{2 d} \cdot \frac{\sqrt{B_{1}} \pm \sqrt{B_{2}}}{\sqrt{h_{A, 1}} \pm \sqrt{h_{A, 2}}}
$$

where the same sign - either plus or minus - simultaneously applies in the numerator and the denominator.

\section{D.3. Deriving condition (D.8)}

Consider a set of offers of type 4 . Its order size $x_{2}$ is determined by one of the two possible expressions in (D.9). We shall assume that $x_{2}$ is defined and $x_{2}>0$. It is then easy to see that the denominator in (D.6) is equal to zero if, and only if, exactly one of the following is true:

i) $B_{1} \neq B_{2}, h_{A, 1} \neq h_{A, 2}$, and

$$
x_{2}=\sqrt{2 d \cdot \frac{B_{1}-B_{2}}{h_{A, 1}-h_{A, 2}}}
$$

ii) $B_{1}=B_{2}, h_{A, 1}=h_{A, 2}$.

Refer to case i). Consider first the order size $x_{2_{1}}$ as defined in (D.9). It satisfies (D.10) whenever

$$
\frac{\sqrt{B_{1}}+\sqrt{B_{2}}}{\sqrt{h_{A, 1}}+\sqrt{h_{A, 2}}}=\sqrt{\frac{B_{1}-B_{2}}{h_{A, 1}-h_{A, 2}}} .
$$

By squaring both sides of the above equality and rearranging the terms, we can rewrite it in the following form:

$$
\left(B_{1}+2 \sqrt{B_{1} B_{2}}+B_{2}\right)\left(h_{A, 1}-h_{A, 2}\right)=\left(h_{A, 1}+2 \sqrt{h_{A, 1} h_{A, 2}}+h_{A, 2}\right)\left(B_{1}-B_{2}\right) .
$$

Expanding the parentheses, rearranging the terms and simplifying gives:

$$
B_{2} h_{A, 1}+\sqrt{B_{1} B_{2}}\left(h_{A, 1}-h_{A, 2}\right)=B_{1} h_{A, 2}+\sqrt{h_{A, 1} h_{A, 2}}\left(B_{1}-B_{2}\right)
$$

Dividing both sides of the equality by $B_{2} h_{A, 2}$ yields:

$$
\frac{h_{A, 1}}{h_{A, 2}}+\sqrt{\frac{B_{1}}{B_{2}}}\left(\frac{h_{A, 1}}{h_{A, 2}}-1\right)=\frac{B_{1}}{B_{2}}+\sqrt{\frac{h_{A, 1}}{h_{A, 2}}}\left(\frac{B_{1}}{B_{2}}-1\right) .
$$

Let $r=B_{1} / B_{2}$ and $s=h_{A, 1} / h_{A, 2}$. Then the above equality can be rewritten as follows:

$$
s-r=\sqrt{s}(r-1)-\sqrt{r}(s-1)
$$


Expanding the parentheses and rearranging the terms on the right gives:

$$
s-r=(\sqrt{r s}+1)(\sqrt{r}-\sqrt{s}) .
$$

It can then be easily seen that the above equality holds if and only if $r=s$.

Conducting the same analysis for the order size $x_{2_{2}}$ as defined by (D.9) and using the same notation, one arrives at the equality

$$
s-r=(\sqrt{r s}+1)(\sqrt{s}-\sqrt{r})
$$

which is obviously satisfied with $r=s$, and otherwise simplifies to

$$
\sqrt{s}+\sqrt{r}=\sqrt{r s}+1
$$

or, equivalently,

$$
\sqrt{s}-1=\sqrt{r}(\sqrt{s}-1) \text {. }
$$

It is easy to see that this equality can only be satisfied with either $r=1$ or $s=1$ or both. However, case i) under consideration assumes that $B_{1} \neq B_{2}$ and $h_{A, 1} \neq h_{A, 2}$, and hence $r, s \neq 1$.

Thus, in case i), a positive order size $x_{2}$ as determined by (D.9) turns the denominator in (D.6) to zero if and only if $r=s$ or, equivalently,

$$
\frac{B_{1}}{B_{2}}=\frac{h_{A, 1}}{h_{A, 2}}
$$

Note that under this condition, the buyer's cost structures $\left\{B_{1}, h_{A, 1}\right\}$ and $\left\{B_{2}, h_{A, 2}\right\}$ strictly dominate one another in Pareto sense; furthermore, both order sizes $x_{2_{1,2}}$ happen to be positive.

Refer now to case ii). In this case, condition (D.11) automatically holds true. Thus, taking both cases i) and ii) into account, we conclude that, given a positive order size $x_{2}$, a zero denominator in (D.6) implies condition (D.11).

Conversely, assume that condition (D.11) holds. It then obviously implies that either $B_{1} \neq B_{2}$, $h_{A, 1} \neq h_{A, 2}$ or $B_{1}=B_{2}, h_{A, 1}=h_{A, 2}$ is true. In the former case, condition (D.11) further implies condition (D.10) to hold, whereas the latter case represents case ii). Hence, condition (D.11) implies either case i) or case ii). We know that either case leads to a zero denominator in (D.6).

Thus, having a zero denominator in (D.6) with a given order size $x_{2}>0$ from a set of offers of type 4 is equivalent to having condition (D.11) satisfied. 


\section{Appendix E. Detailed derivation of KKT solutions of type 6}

KKT solutions of type 6 are associated with the case $\lambda_{1}, \lambda_{2}, \mu_{1}, \mu_{2}>0$ (Sucky, 2006, p. 534). Their derivation is similar to that of KKT solutions of type 4, see Appendix D above. Specifically, from (A.5) and (A.6) we derive:

$$
z_{i}=K_{i}^{A}\left(x_{i}\right)-K_{i}^{A}\left(x_{A, i}^{*}\right) \quad(i=1,2) .
$$

Substituting this into (A.7) and (A.8), we obtain the expressions of order sizes $x_{1}, x_{2}$ as given in equations (36), (37) in Sucky (2006), which we subsequently simplify in accordance with Section D.2 of Appendix D to:

$$
x_{1,2_{1,2}}=\sqrt{2 d} \cdot \frac{\sqrt{B_{1}} \pm \sqrt{B_{2}}}{\sqrt{h_{A, 1}} \pm \sqrt{h_{A, 2}}} .
$$

From (A.3) and (A.4) we respectively have:

$$
\begin{aligned}
& \lambda_{1}+\mu_{1}=\omega_{1}+\mu_{2} \\
& \lambda_{2}+\mu_{2}=\omega_{2}+\mu_{1}
\end{aligned}
$$

By substituting the right-hand sides of the above equations for their left-hand sides into (A.1) and (A.2) respectively, we derive expressions of $\mu_{1}$ and $\mu_{2}$ in the exact same form (B.6) and (B.9), respectively. If both values are defined, we need to require from them to be nonnegative, and further that

$$
-\omega_{2} \leq \mu_{1}-\mu_{2} \leq \omega_{1}
$$

in order to ensure nonnegativity of Lagrange multipliers $\lambda_{1}$ and $\lambda_{2}$ as per (A.3) and (A.4). Otherwise, both values given by (B.6) and (B.9) are undefined. This happens if and only if $x_{A, 1}^{*}=x_{A, 2}^{*}$ (provided that $x_{1}, x_{2}>0$ ), see Section D.1 of Appendix D. In such case, conditions (A.1) and (A.2) hold if and only if $x_{1}=\mathrm{JELS}_{1}$ and $x_{2}=\mathrm{JELS}_{2}$ respectively. This implies the following necessary optimality condition for the sets of offers expressed by (E.1) and (E.2):

$$
\begin{cases}x_{1}=\mathrm{JELS}_{1}, x_{2}=\mathrm{JELS}_{2}, & \text { if } x_{A, 1}^{*}=x_{A, 2}^{*} \\ \mu_{1} \geq 0, \mu_{2} \geq 0,-\omega_{2} \leq \mu_{1}-\mu_{2} \leq \omega_{1}, & \text { otherwise }\end{cases}
$$

where $\mu_{1}$ and $\mu_{2}$ are as defined in (B.6) and (B.9), respectively. There are no feasibility conditions (other than $x_{1}, x_{2}>0$ ) to be verified for these sets of offers. 


\section{Appendix F. Detailed derivation of KKT solutions of type 7}

KKT solutions of type 7 assume $\lambda_{1}=0$ and $\lambda_{2}, \mu_{1}, \mu_{2}>0$. Further, KKT conditions (A.3) and (A.4) respectively require:

$$
\begin{aligned}
& \omega_{1}-\lambda_{1}-\mu_{1}+\mu_{2}=0, \\
& \omega_{2}-\lambda_{2}+\mu_{1}-\mu_{2}=0 .
\end{aligned}
$$

Adding these two equalities together and noting that $\lambda_{1}=0$, we immediately obtain $\lambda_{2}=1$. The first equality accordingly yields:

$$
\mu_{1}=\mu_{2}+\omega_{1}
$$

By substituting $\lambda_{1}=0, \lambda_{2}=1$ into conditions (A.1) and (A.2), we can rewrite them respectively as:

$$
\begin{aligned}
& \omega_{1} \frac{d K^{P}\left(x_{1}\right)}{d x_{1}}+\mu_{1} \frac{d K_{1}^{A}\left(x_{1}\right)}{d x_{1}}-\mu_{2} \frac{d K_{2}^{A}\left(x_{1}\right)}{d x_{1}}=0 \\
& \omega_{2} \frac{d K^{P}\left(x_{2}\right)}{d x_{2}}+\frac{d K_{2}^{A}\left(x_{2}\right)}{d x_{2}}-\mu_{1} \frac{d K_{1}^{A}\left(x_{2}\right)}{d x_{2}}+\mu_{2} \frac{d K_{2}^{A}\left(x_{2}\right)}{d x_{2}}=0
\end{aligned}
$$

Substituting (F.1) and $\omega_{1}=1-\omega_{2}$ into (F.2) and (F.3) respectively yields:

$$
\begin{aligned}
& \omega_{1} \cdot\left(\frac{d K^{P}\left(x_{1}\right)}{d x_{1}}+\frac{d K_{1}^{A}\left(x_{1}\right)}{d x_{1}}\right)=\mu_{2} \cdot\left(\frac{d K_{2}^{A}\left(x_{1}\right)}{d x_{1}}-\frac{d K_{1}^{A}\left(x_{1}\right)}{d x_{1}}\right) \\
& \omega_{2} \cdot\left(\frac{d K^{P}\left(x_{2}\right)}{d x_{2}}+\frac{d K_{1}^{A}\left(x_{2}\right)}{d x_{2}}\right)=\left(1+\mu_{2}\right) \cdot\left(\frac{d K_{1}^{A}\left(x_{2}\right)}{d x_{2}}-\frac{d K_{2}^{A}\left(x_{2}\right)}{d x_{2}}\right)
\end{aligned}
$$

Suppose first that the parenthetical expression on the right in (F.4) is equal to zero. This implies - by the definition of the cost functions involved - that

$$
x_{1}^{2}=2 d \cdot \frac{B_{1}-B_{2}}{h_{A, 1}-h_{A, 2}} .
$$

We now below show that (F.6) further implies $x_{1}=x_{2}$ to hold. Indeed, refer to KKT conditions (A.7)-(A.8). Since $\mu_{1}, \mu_{2}>0$ holds by assumption, (A.7)-(A.8) respectively require the following equalities to hold:

$$
\begin{aligned}
& K_{1}^{A}\left(x_{2}\right)-z_{2}-K_{1}^{A}\left(x_{1}\right)+z_{1}=0 \\
& K_{2}^{A}\left(x_{1}\right)-z_{1}-K_{2}^{A}\left(x_{2}\right)+z_{2}=0 .
\end{aligned}
$$


Adding both equalities together yields:

$$
K_{1}^{A}\left(x_{1}\right)-K_{2}^{A}\left(x_{1}\right)=K_{1}^{A}\left(x_{2}\right)-K_{2}^{A}\left(x_{2}\right)
$$

By definition of the cost functions involved, (F.7) can be rewritten as

$$
\left(B_{1}-B_{2}\right) \cdot\left(\frac{d}{x_{2}}-\frac{d}{x_{1}}\right)=\frac{1}{2}\left(h_{A, 1}-h_{A, 2}\right) \cdot\left(x_{1}-x_{2}\right) .
$$

If $x_{1}=x_{2}$ then (F.8) is obviously satisfied, otherwise $x_{1}$ and $x_{2}$ must obey:

$$
x_{1} x_{2}=\mathcal{A}
$$

where $\mathcal{A}$ denotes the right-hand side of (F.6). Note that (F.9) has to be satisfied by any set of offers of type 7 , what necessarily requires $\mathcal{A}>0$. We will accordingly assume this to hold throughout this section. Note further that this in turn implies that the buyer's possible cost structures strictly dominate one another in Pareto sense.

Given (F.6), it is then immediate to see that equation (F.9) can only be satisfied with $x_{1}=x_{2}$. Furthermore, this immediately implies that the parenthetical expression on the right of (F.5) equals to zero as well, and vice versa. Thus the parenthetical expressions on the right of (F.4) and (F.5) must be either both non-zero or both zero; the latter case holds if and only if $x_{1}=x_{2}=\sqrt{\mathcal{A}}$. In that case, equalities (F.4) and (F.5) hold if and only if

$$
x_{1}=x_{2}=\mathrm{JELS}_{1}
$$

because $\mathrm{JELS}_{1}$ is the unique order size that minimizes the joint total cost function $K^{P}(x)+K_{1}^{A}(x)$ and thus turns its derivative on the left in (F.4) and (F.5) to zero. Then, accordingly, equalities (F.4) and (F.5) are fulfilled with any feasible value of $\mu_{2}$; by the same argument as in Section D.1 of Appendix D, we can set $\mu_{2}=0$. By (F.1), this implies $\mu_{1}=\omega_{1}>0$.

It is worth noting that condition (F.10) further implies that $\mathrm{JELS}_{1}=\mathrm{JELS}_{2}$. Indeed, by substituting $\mu_{2}=\mu_{1}-\omega_{1}$ due to (F.1) into (F.4), it is straightforward to rewrite the latter in the following form:

$$
\omega_{1} \cdot\left(\frac{d K^{P}\left(x_{1}\right)}{d x_{1}}+\frac{d K_{2}^{A}\left(x_{1}\right)}{d x_{1}}\right)=\mu_{1} \cdot\left(\frac{d K_{2}^{A}\left(x_{1}\right)}{d x_{1}}-\frac{d K_{1}^{A}\left(x_{1}\right)}{d x_{1}}\right) .
$$

Since the solution in question turns the right-hand side in the above equation to zero, the parenthetical expression in the left-hand side must be equal to zero, as well - what in turn implies that $x_{1}$ minimizes the joint total costs associated with the buyer's cost structure $\left\{B_{2}, h_{A, 2}\right\}$ and 
thus coincides with the respective joint economic lot size $\mathrm{JELS}_{2}$. Hence both JELS 1 and JELS 2 are equal to $x_{1}$ and therefore to each other.

Consider now the case of $x_{1} \neq x_{2}$. Then, by the above argument, the parenthetical expressions on the right of (F.4) and (F.5) are non-zero. Thus (F.4) and (F.5) respectively yield the following expressions for $\mu_{2}$ :

$$
\begin{aligned}
& \mu_{2}=\omega_{1} \cdot\left(\frac{d K^{P}\left(x_{1}\right)}{d x_{1}}+\frac{d K_{1}^{A}\left(x_{1}\right)}{d x_{1}}\right) /\left(\frac{d K_{2}^{A}\left(x_{1}\right)}{d x_{1}}-\frac{d K_{1}^{A}\left(x_{1}\right)}{d x_{1}}\right) \\
& \mu_{2}=\omega_{2} \cdot\left(\frac{d K^{P}\left(x_{2}\right)}{d x_{2}}+\frac{d K_{1}^{A}\left(x_{2}\right)}{d x_{2}}\right) /\left(\frac{d K_{1}^{A}\left(x_{2}\right)}{d x_{2}}-\frac{d K_{2}^{A}\left(x_{2}\right)}{d x_{2}}\right)-1
\end{aligned}
$$

Refer first to (F.11). By (F.9), $x_{1}=\mathcal{A} / x_{2}$, where $\mathcal{A}$ represents the right-hand side of (F.6). Then, by definition of the cost functions involved, it is straightforward to rewrite the parenthetical expression in the numerator of (F.11) in the following form:

$$
\frac{1}{2} \cdot\left[\frac{d}{p} h_{P}+h_{A, 1}-2 d\left(R+B_{1}\right) \frac{x_{2}^{2}}{\mathcal{A}^{2}}\right] .
$$

Similarly, the denominator of (F.11) takes the form:

$$
\frac{1}{2} \cdot\left[h_{A, 2}-h_{A, 1}+2 d\left(B_{1}-B_{2}\right) \frac{x_{2}^{2}}{\mathcal{A}^{2}}\right]=\frac{1}{2 \mathcal{A}} \cdot\left[\left(h_{A, 1}-h_{A, 2}\right) x_{2}^{2}-2 d\left(B_{1}-B_{2}\right)\right] .
$$

Accordingly, (F.11) simplifies to:

$$
\mu_{2}=\frac{\left(\frac{d}{p} h_{P}+h_{A, 1}\right) \cdot \mathcal{A}-2 d\left(R+B_{1}\right) x_{2}^{2} / \mathcal{A}}{\left(h_{A, 1}-h_{A, 2}\right) x_{2}^{2}-2 d\left(B_{1}-B_{2}\right)} \cdot \omega_{1} .
$$

By definition of the cost functions involved, it is further straightforward to rewrite (F.12) in the following form:

$$
\mu_{2}=\frac{\left(\frac{d}{p} h_{P}+h_{A, 1}\right) x_{2}^{2}-2 d\left(R+B_{1}\right)}{\left(h_{A, 1}-h_{A, 2}\right) x_{2}^{2}-2 d\left(B_{1}-B_{2}\right)} \cdot \omega_{2}-1
$$

Note that the fractions in (F.13) and (F.14) have a common denominator which is non-zero due to the assumptions $\mathcal{A}>0, x_{1} \neq x_{2}$ and equation (F.9). Setting now both expressions for $\mu_{2}$ equal to each other yields the following equation:

$$
\begin{aligned}
& \omega_{1} \cdot\left[\left(\frac{d}{p} h_{P}+h_{A, 1}\right) \cdot \mathcal{A}-2 d\left(R+B_{1}\right) x_{2}^{2} \cdot \frac{1}{\mathcal{A}}\right]= \\
= & \omega_{2} \cdot\left[\left(\frac{d}{p} h_{P}+h_{A, 1}\right) x_{2}^{2}-2 d\left(R+B_{1}\right)\right]+2 d\left(B_{1}-B_{2}\right)-\left(h_{A, 1}-h_{A, 2}\right) x_{2}^{2} .
\end{aligned}
$$


Solving this equation for $x_{2}^{2}$ yields:

$$
x_{2}^{2}=\frac{\omega_{1} \cdot\left(\frac{d}{p} h_{P}+h_{A, 1}\right)+\omega_{2} \cdot 2 d\left(R+B_{1}\right) \cdot \frac{1}{\mathcal{A}}+h_{A, 2}-h_{A, 1}}{\omega_{2} \cdot\left(\frac{d}{p} h_{P}+h_{A, 1}\right)+\omega_{1} \cdot 2 d\left(R+B_{1}\right) \cdot \frac{1}{\mathcal{A}}+h_{A, 2}-h_{A, 1}} \cdot \mathcal{A} .
$$

Thus $x_{2}$ is determined as the square root of the right-hand side in (F.15) - provided that it is defined, and $x_{1}$ is determined due to (F.9) as $x_{1}=\mathcal{A} / x_{2}$. It is straightforward to rewrite $x_{1}$ in the form given in equation (C.4). Note that $\omega_{1}=\omega_{2}$ would make the numerator and the denominator in (F.15) equal and lead to $x_{1}=x_{2}=\sqrt{\mathcal{A}}$. Hence $x_{1} \neq x_{2}$ is only possible to hold when $\omega_{1} \neq \omega_{2}$. Note further that the value of $\mu_{2}$ can be determined using equation (F.14). It has been assumed to be positive; however, by the same argument as in Section D.1 of Appendix D, it is sufficient to require $\mu_{2} \geq 0$. Then by (F.1), $\mu_{1}=\mu_{2}+\omega_{1}>0$. In particular, it is easy to see that by virtue of (F.13) and (F.14), $\mu_{2}=0$ makes the solution in question coincide with the set of offers of type 3 .

We now determine the side payments $z_{1}, z_{2}$ to be respectively offered in conjunction with the order sizes $x_{1}, x_{2}$. With $\lambda_{2}=1$, KKT condition (A.6) yields the side payment

$$
z_{2}=K_{2}^{A}\left(x_{2}\right)-K_{2}^{A}\left(x_{A, 2}^{*}\right)
$$

which is obviously nonnegative. With $\mu_{1}>0$, KKT condition (A.7) yields:

$$
z_{1}=K_{1}^{A}\left(x_{1}\right)-K_{1}^{A}\left(x_{2}\right)+z_{2}
$$

Note further that (F.9) implies (F.7) to hold. This allows to rewrite (F.17) as

$$
z_{1}=K_{2}^{A}\left(x_{1}\right)-K_{2}^{A}\left(x_{2}\right)+z_{2}
$$

This obviously satisfies (A.8) for any $\mu_{2} \geq 0$. Further, substituting (F.16) into (F.18) gives:

$$
z_{1}=K_{2}^{A}\left(x_{1}\right)-K_{2}^{A}\left(x_{2}\right)+K_{2}^{A}\left(x_{2}\right)-K_{2}^{A}\left(x_{A, 2}^{*}\right)=K_{2}^{A}\left(x_{1}\right)-K_{2}^{A}\left(x_{A, 2}^{*}\right)
$$

and hence proves $z_{1}$ to be nonnegative. With $\lambda_{1}=0$ by assumption, KKT condition (A.5) requires

$$
K_{1}^{A}\left(x_{A, 1}^{*}\right)-K_{1}^{A}\left(x_{1}\right)+z_{1} \geq 0
$$

to ensure the feasibility of solution in question. Substituting (F.17) into (F.20) finally yields:

$$
K_{1}^{A}\left(x_{2}\right)-z_{2} \leq K_{1}^{A}\left(x_{A, 1}^{*}\right)
$$




\section{Appendix G. Proof of Proposition 1}

We shall prove that satisfying KKT conditions (A.1)-(A.8) is necessary for local optimality of a set of offers $\left(x_{1}, z_{1}, x_{2}, z_{2}\right)$ to problem (8)-(12). For this purpose we will resort to the MangasarianFromovitz constraint qualification (Mangasarian and Fromovitz, 1967), as follows. Let a problem

$$
\min f(x) \text { s.t. } g_{i}(x) \leq 0, \quad i=1, \ldots, m, \quad x \in X \subset \mathbb{R}^{n}, X \text { open }
$$

be given. Then the following proposition follows immediately from Theorem 7.3.7 in Mangasarian (1969) and Theorem 4.10.4 in Hestenes (1975). ${ }^{9}$

Proposition G.1. Let $\bar{x}$ be a feasible solution of problem (P), and denote $I=\left\{i \mid g_{i}(\bar{x})=0\right\}$. Suppose that $f$ and $g_{i}, i=1, \ldots, m$, are differentiable at $\bar{x}$. Furthermore, suppose that $\nabla g_{i}(\bar{x})$, $i \in I$, are positively linearly independent - i.e., for $u_{i} \geq 0, i \in I$, the equality

$$
\sum_{i \in I} u_{i} \nabla g_{i}(\bar{x})=0
$$

can only hold if $u_{i}=0$ for all $i \in I$. If $\bar{x}$ solves (P) locally, there exist scalars $\lambda_{i} \geq 0$ for $i=1, \ldots, m$ such that

$$
\nabla f(\bar{x})+\sum_{i=1}^{m} \lambda_{i} \nabla g_{i}(\bar{x})=0 \quad \text { and } \quad \lambda_{i} g_{i}(\bar{x})=0, \quad i=1, \ldots, m
$$

Let now $\left(\bar{x}_{1}, \bar{z}_{1}, \bar{x}_{2}, \bar{z}_{2}\right)$ be a feasible set of offers that delivers a local optimum to problem (8)-(12). To simplify the subsequent exposition, denote

$$
K_{A, i}^{\prime}(x) \equiv \frac{d K_{i}^{A}(x)}{d x}
$$

Consider the Jacobian matrix of constraints $(9)-(12)$ at $\left(\bar{x}_{1}, \bar{z}_{1}, \bar{x}_{2}, \bar{z}_{2}\right)$ :

$$
J=\left(\begin{array}{rrrr}
K_{A, 1}^{\prime}\left(\bar{x}_{1}\right) & -1 & 0 & 0 \\
0 & 0 & K_{A, 2}^{\prime}\left(\bar{x}_{2}\right) & -1 \\
K_{A, 1}^{\prime}\left(\bar{x}_{1}\right) & -1 & -K_{A, 1}^{\prime}\left(\bar{x}_{2}\right) & 1 \\
-K_{A, 2}^{\prime}\left(\bar{x}_{1}\right) & 1 & K_{A, 2}^{\prime}\left(\bar{x}_{2}\right) & -1
\end{array}\right)
$$

\footnotetext{
${ }^{9}$ The notation and the wording partly used in Proposition G.1 are due to Bazaraa et al. (2006, Theorem 4.2.13). The hypothesis of the proposition involves an equivalent form of the Mangasarian-Fromovitz constraint qualification as per Theorem 4.10.4 in Hestenes (1975) (see Horsley and Wrobel, 2004, Lemma 14); see also Solodov (2011). In the absence of equality constraints, the Mangasarian-Fromovitz constraint qualification implies the Arrow-HurwiczUzawa constraint qualification (Mangasarian, 1969, p. 102). By Theorem 7.3.7 in Mangasarian (1969), this leads to the assertion of Proposition G.1.
} 
The rows of $J$ represent the gradients of constraints (9)-(12) at $\left(\bar{x}_{1}, \bar{z}_{1}, \bar{x}_{2}, \bar{z}_{2}\right)$; let $J_{i j}$ represent the element in the $i$-th row and $j$-th column of $J$, where $i, j=1, \ldots, 4$. Suppose that there exist $u_{1}, \ldots, u_{4} \geq 0$ such that the equality

$$
u_{1} J_{1 j}+\ldots+u_{4} J_{4 j}=0
$$

holds for each $j=1, \ldots, 4$. Rewriting (G.3) for $j=2,4$ in the explicit form respectively gives:

$$
\begin{aligned}
& -u_{1}-u_{3}+u_{4}=0 \\
& -u_{2}+u_{3}-u_{4}=0
\end{aligned}
$$

Adding these two equalities together yields $-u_{1}-u_{2}=0$, what obviously implies $u_{1}=u_{2}=0$ and, consequently, $u_{3}=u_{4}$. Then (G.3) can be satisfied for $j=1,3$ if and only if the following holds:

$$
\begin{aligned}
& K_{A, 1}^{\prime}\left(\bar{x}_{1}\right)=K_{A, 2}^{\prime}\left(\bar{x}_{1}\right), \\
& K_{A, 2}^{\prime}\left(\bar{x}_{2}\right)=K_{A, 1}^{\prime}\left(\bar{x}_{2}\right) .
\end{aligned}
$$

Thus, if (G.6) and (G.7) do not hold simultaneously, then the gradients of constraints (9)-(12) are positively linearly independent at $\left(\bar{x}_{1}, \bar{z}_{1}, \bar{x}_{2}, \bar{z}_{2}\right)$ in their totality, and so in particular are the gradients of the binding constraints. Hence solution $\left(\bar{x}_{1}, \bar{z}_{1}, \bar{x}_{2}, \bar{z}_{2}\right)$ of problem (8)-(12) satisfies the hypothesis of Proposition G.1. Therefore,

a) it satisfies conditions (G.1) with some $\lambda_{i} \geq 0, i=1, \ldots, 4$.

Furthermore, by assumption,

b) it represents a feasible solution to problem (8)-(12).

Satisfying a) and b) is at the same time equivalent to satisfying KKT conditions (A.1)-(A.8) subject to constraints $x_{1}, x_{2}>0$ (see also Bazaraa et al., 2006, pp. 209-210).

Assume now that (G.6), (G.7) both hold. By definition of the cost functions involved, this is equivalent to having

$$
\bar{x}_{1}=\bar{x}_{2}=\sqrt{2 d \cdot \frac{B_{1}-B_{2}}{h_{A, 1}-h_{A, 2}}} .
$$

Note that this is possible only if the buyer's cost structures strictly dominate one another in Pareto sense - i.e., if either $B_{1}>B_{2}, h_{A, 1}>h_{A, 2}$ or $B_{1}<B_{2}, h_{A, 1}<h_{A, 2}$ holds. Then, due to the equality of $\bar{x}_{1}$ and $\bar{x}_{2}$, constraints (11)-(12) imply $\bar{z}_{1}=\bar{z}_{2}$; note that both these constraints are 
binding at $\left(\bar{x}_{1}, \bar{z}_{1}, \bar{x}_{2}, \bar{z}_{2}\right)$. To comply with constraints (9)-(10), the side payments have to satisfy

$$
\bar{z}_{1}=\bar{z}_{2} \geq \max \left\{K_{1}^{A}\left(\bar{x}_{1}\right)-K_{1}^{A}\left(x_{A, 1}^{*}\right), K_{2}^{A}\left(\bar{x}_{2}\right)-K_{2}^{A}\left(x_{A, 2}^{*}\right)\right\} .
$$

However, the above non-strict inequality must obviously hold as equality:

$$
\bar{z}_{1}=\bar{z}_{2}=\max \left\{K_{1}^{A}\left(\bar{x}_{1}\right)-K_{1}^{A}\left(x_{A, 1}^{*}\right), K_{2}^{A}\left(\bar{x}_{2}\right)-K_{2}^{A}\left(x_{A, 2}^{*}\right)\right\}
$$

since it would otherwise contradict the local optimality of the set of offers in question. Furthermore, assume that of the two differences on the right in (G.10), the first one happens to be maximal so that, in particular, the following holds:

$$
\bar{z}_{1}=K_{1}^{A}\left(\bar{x}_{1}\right)-K_{1}^{A}\left(x_{A, 1}^{*}\right)
$$

With $\bar{x}_{1}=\bar{x}_{2}$ and $\bar{z}_{1}=\bar{z}_{2}$, objective function (1) can obviously be expressed at $\left(\bar{x}_{1}, \bar{z}_{1}, \bar{x}_{2}, \bar{z}_{2}\right)$ as

$$
K^{P}\left(\bar{x}_{1}\right)+\bar{z}_{1} .
$$

It is then easy to see that the lot size $\bar{x}_{1}$ has to satisfy:

$$
\bar{x}_{1}=\mathrm{JELS}_{1}
$$

because the respective joint economic lot size is known to minimize (G.12) given (G.11) (cf. Banerjee, 1986; Sucky, 2006), so that violating (G.13) would obviously contradict the local optimality of the set of offers in question: indeed, shifting the lot size $\bar{x}_{1}=\bar{x}_{2}$ in the direction of JELS $S_{1}$ by an infinitesimal amount $\delta$ and obtaining $\bar{z}_{1}, \bar{z}_{2}$ by means of (G.10) would lead to a feasible set of offers that satisfies constraints (9)-(10) due to (G.9) and constraints (11)-(12) due to $\bar{x}_{1}=\bar{x}_{2}$ and $\bar{z}_{1}=\bar{z}_{2}$, while delivering a lower value to the objective function in (G.12).

It can then be immediately seen that the set of offers $\left(\bar{x}_{1}, \bar{z}_{1}, \bar{x}_{2}, \bar{z}_{2}\right)$ defined by (G.8), (G.10) represents a set of offers of type 8 (see Table 1 ) that satisfies the respective feasibility condition due to (G.10) and the equality $\bar{x}_{1}=\bar{x}_{2}$, and satisfies the necessary optimality condition as per (G.13). Since all sets of offers of types 1 to 8 and their feasibility and necessary optimality conditions are derived from KKT conditions (A.1)-(A.8), we therefore conclude that the set of offers in question necessarily satisfies them. Also, by construction, is satisfies constraints $z_{1}, z_{2} \geq 0$ and $x_{1}, x_{2}>0$.

By using the same approach when the right-hand side in (G.10) is equal to $K_{2}^{A}\left(\bar{x}_{2}\right)-K_{2}^{A}\left(x_{A, 2}^{*}\right)$, we can show that the set of offers in question coincides with a set of offers of type 7 and therefore satisfies KKT conditions (A.1)-(A.8) and constraints $z_{1}, z_{2} \geq 0, x_{1}, x_{2}>0$ too. 


\section{Appendix H. Proof of Proposition 2}

Let $\left(\bar{x}_{1}, \bar{z}_{1}, \bar{x}_{2}, \bar{z}_{2}\right)$ represent an optimal solution of problem (1)-(6) for some $s \in S$. Let further $S^{*}:=\{1,2,3,4 \mathrm{a}-\mathrm{b}, 5 \mathrm{a}-\mathrm{b}, 7 \mathrm{a}, 8 \mathrm{a}\} \subset S$.

Referring to Table 1, we can immediately see that for $s \in S^{*}$, it holds either by construction or by virtue of necessary optimality conditions that $\bar{x}_{i}=\operatorname{JELS}_{i}$ for at least one $i \in\{1,2\}$. We shall below prove that for $s \in S \backslash S^{*}=\{6 \mathrm{a}-\mathrm{d}, 7 \mathrm{~b}, 8 \mathrm{~b}\}$, it must also necessarily hold that $\bar{x}_{i}=\mathrm{JELS}_{i}$ for at least one $i \in\{1,2\}$.

To this end, refer to the feasibility conditions of the KKT solution of type 1, see Table 1. It is easy to rewrite them in the following form:

$$
\bar{K}\left(\mathrm{JELS}_{1}\right) \leq K_{1}^{A}\left(x_{A, 1}^{*}\right)-K_{2}^{A}\left(x_{A, 2}^{*}\right) \leq \bar{K}\left(\mathrm{JELS}_{2}\right),
$$

where

$$
\bar{K}(x)=K_{1}^{A}(x)-K_{2}^{A}(x)
$$

It is also straightforward to verify that the inequality $\bar{K}\left(\mathrm{JELS}_{1}\right) \leq \bar{K}\left(\mathrm{JELS}_{2}\right)$ always holds by definition of the joint economic lot sizes JELS $1, \mathrm{JELS}_{2}$ in (7). Thus at most one of the two inequalities in (H.1) can be violated. If none is violated then the set of offers of type 1 proves to be a feasible solution of problem (1)-(6). Furthermore, we can easily verify that it uniquely minimizes the objective function (1) subject to individually rationality constraints (2)-(3) (cf. Sucky, 2006, Section 4.2). Therefore, it must represent a unique optimal solution of problem (1)-(6) as well. Hence, an optimal set of offers $\left(\bar{x}_{1}, \bar{z}_{1}, \bar{x}_{2}, \bar{z}_{2}\right)$ must necessarily coincide with the set of offers of type $1-$ so that $\bar{x}_{i}=\operatorname{JELS}_{i}$ holds for $i=1,2$. Otherwise, if one of the inequalities in (H.1) is violated, distinguish between the following three cases.

Case 1. Let $s \in\{6 \mathrm{a}-\mathrm{d}\}$. Assume that the $1^{\text {st }}$ inequality in (H.1) holds, while the $2^{\text {nd }}$ does not. We will prove that $\bar{x}_{1}=\mathrm{JELS}_{1}$. Assume the opposite: $\bar{x}_{1} \neq \mathrm{JELS}_{1}$. By construction, a set of offers of type 6 satisfies constraints (2)-(5) as equalities (see Section B.3 in Appendix B), so that:

$$
\begin{aligned}
& K_{1}^{A}\left(\bar{x}_{1}\right)-\bar{z}_{1}=K_{1}^{A}\left(x_{A, 1}^{*}\right) \\
& K_{2}^{A}\left(\bar{x}_{2}\right)-\bar{z}_{2}=K_{2}^{A}\left(x_{A, 2}^{*}\right) \\
& K_{1}^{A}\left(\bar{x}_{1}\right)-\bar{z}_{1}=K_{1}^{A}\left(\bar{x}_{2}\right)-\bar{z}_{2} \\
& K_{2}^{A}\left(\bar{x}_{2}\right)-\bar{z}_{2}=K_{2}^{A}\left(\bar{x}_{1}\right)-\bar{z}_{1}
\end{aligned}
$$


Consider an alternative set of offers $\left(\tilde{x}_{1}, \tilde{z}_{1}, \bar{x}_{2}, \bar{z}_{2}\right)$ where

$$
\begin{aligned}
& \tilde{x}_{1}=\mathrm{JELS}_{1}, \\
& \tilde{z}_{1}=K_{1}^{A}\left(\tilde{x}_{1}\right)-K_{1}^{A}\left(x_{A, 1}^{*}\right) .
\end{aligned}
$$

We below prove the feasibility of this set of offers. Indeed, it satisfies constraints (2), (3) due to equations (H.8), (H.4) respectively. Further, equations (H.3) and (H.8) imply:

$$
K_{1}^{A}\left(\bar{x}_{1}\right)-\bar{z}_{1}=K_{1}^{A}\left(\tilde{x}_{1}\right)-\tilde{z}_{1}
$$

and therefore constraint (4) holds for the set of offers in question by virtue of (H.5). Further, the $1^{\text {st }}$ inequality in (H.1) holds by assumption; we can obviously rewrite it by virtue of (H.2) and (H.7) as follows:

$$
K_{1}^{A}\left(\tilde{x}_{1}\right)-K_{2}^{A}\left(\tilde{x}_{1}\right) \leq K_{1}^{A}\left(x_{A, 1}^{*}\right)-K_{2}^{A}\left(x_{A, 2}^{*}\right)
$$

Rearranging the terms above gives:

$$
K_{2}^{A}\left(x_{A, 2}^{*}\right) \leq K_{2}^{A}\left(\tilde{x}_{1}\right)-\left(K_{1}^{A}\left(\tilde{x}_{1}\right)-K_{1}^{A}\left(x_{A, 1}^{*}\right)\right)
$$

what can be rewritten by virtue of (H.4) and (H.8) as follows:

$$
K_{2}^{A}\left(\bar{x}_{2}\right)-\bar{z}_{2} \leq K_{2}^{A}\left(\tilde{x}_{1}\right)-\tilde{z}_{1}
$$

what implies that constraint (5) holds for the set of offers in question. Thus, this set of offers proves to be feasible. Furthermore, it delivers a lower value to the objective function (1) than the original set of offers $\left(\bar{x}_{1}, \bar{y}_{1}, \bar{x}_{2}, \bar{y}_{2}\right)$ does because the order size $\tilde{x}_{1}$ and the side payment $\tilde{z}_{1}$ defined in (H.7)-(H.8) uniquely minimize

$$
K^{P}\left(x_{1}\right)+z_{1} \quad \text { s.t. } \quad K_{1}^{A}\left(x_{1}\right)-z_{1} \leq K_{1}^{A}\left(x_{A, 1}^{*}\right)
$$

(see Sucky, 2006, Section 4.2). But this contradicts the optimality of the original set of offers $\left(\bar{x}_{1}, \bar{y}_{1}, \bar{x}_{2}, \bar{y}_{2}\right)$, and hence $\bar{x}_{1}=\mathrm{JELS}_{1}$ must indeed hold true.

We proceed similarly when the $1^{\text {st }}$ inequality in (H.1) does not hold, while the $2^{\text {nd }}$ does. It is easy to see that in either event, the given set of offers $\left(\bar{x}_{1}, \bar{y}_{1}, \bar{x}_{2}, \bar{y}_{2}\right)$ coincides with a set of offers of type 4 or 5 . 
Case 2. Let $s=7 \mathrm{~b}$. Assume that the $1^{\text {st }}$ inequality in (H.1) holds, while the $2^{\text {nd }}$ does not - i.e.:

$$
\bar{K}\left(\mathrm{JELS}_{1}\right) \leq K_{1}^{A}\left(x_{A, 1}^{*}\right)-K_{2}^{A}\left(x_{A, 2}^{*}\right)>\bar{K}\left(\mathrm{JELS}_{2}\right)
$$

It is easy to verify that $\bar{K}\left(\mathrm{JELS}_{1}\right) \leq \bar{K}\left(\mathrm{JELS}_{2}\right)$ holds by virtue of (H.2) and by definition of joint economic lot sizes JELS 1,2 in (7). We can thus rewrite the above in the following form:

$$
\bar{K}\left(\mathrm{JELS}_{1}\right) \leq \bar{K}\left(\mathrm{JELS}_{2}\right)<K_{1}^{A}\left(x_{A, 1}^{*}\right)-K_{2}^{A}\left(x_{A, 2}^{*}\right)
$$

Let $\Delta$ represent the difference between the right and left-hand sides in (H.9):

$$
\Delta=K_{1}^{A}\left(x_{A, 1}^{*}\right)-K_{2}^{A}\left(x_{A, 2}^{*}\right)-\bar{K}\left(\operatorname{JELS}_{1}\right)
$$

Obviously, $\Delta>0$. Consider now the given set of offers $\left(\bar{x}_{1}, \bar{z}_{1}, \bar{x}_{2}, \bar{z}_{2}\right)$. Since it represents a set of offers of type 7 , the following holds for it by construction (see Appendix F):

$$
\begin{aligned}
& K_{1}^{A}\left(\bar{x}_{1}\right)-\bar{z}_{1} \leq K_{1}^{A}\left(x_{A, 1}^{*}\right) \\
& K_{2}^{A}\left(\bar{x}_{2}\right)-\bar{z}_{2}=K_{2}^{A}\left(x_{A, 2}^{*}\right) \\
& K_{1}^{A}\left(\bar{x}_{1}\right)-\bar{z}_{1}=K_{1}^{A}\left(\bar{x}_{2}\right)-\bar{z}_{2} \\
& K_{2}^{A}\left(\bar{x}_{2}\right)-\bar{z}_{2}=K_{2}^{A}\left(\bar{x}_{1}\right)-\bar{z}_{1}
\end{aligned}
$$

Let

$$
\delta=K_{1}^{A}\left(x_{A, 1}^{*}\right)-\left(K_{1}^{A}\left(\bar{x}_{1}\right)-\bar{z}_{1}\right)
$$

It obviously holds that $\delta \geq 0$ as per (H.11). We distinguish between the following two sub-cases.

1) Assume that $\delta \leq \Delta$. We will prove that $\bar{x}_{1}=\operatorname{JELS}_{1}$. Assume the opposite: that $\bar{x}_{1} \neq \mathrm{JELS}_{1}$, and consider an alternative set of offers $\left(\tilde{x}_{1}, \tilde{z}_{1}, \bar{x}_{2}, \bar{z}_{2}\right)$ where

$$
\begin{aligned}
& \tilde{x}_{1}=\operatorname{JELS}_{1}, \\
& \tilde{z}_{1}=K_{1}^{A}\left(\tilde{x}_{1}\right)-K_{1}^{A}\left(x_{A, 1}^{*}\right)+\delta .
\end{aligned}
$$

The alternative set of offers satisfies constraints (2), (3) due to equations (H.17), (H.12) respectively. Further, equation (H.17) implies:

$$
K_{1}^{A}\left(\bar{x}_{1}\right)-\bar{z}_{1}=K_{1}^{A}\left(\tilde{x}_{1}\right)-\tilde{z}_{1},
$$


and therefore constraint (4) holds for the set of offers in question by virtue of (H.13). Furthermore, we can obviously rewrite (H.10) due to (H.2) and (H.16) as follows:

$$
K_{1}^{A}\left(\tilde{x}_{1}\right)-K_{2}^{A}\left(\tilde{x}_{1}\right)+\Delta=K_{1}^{A}\left(x_{A, 1}^{*}\right)-K_{2}^{A}\left(x_{A, 2}^{*}\right) .
$$

Rearranging the terms above gives:

$$
K_{2}^{A}\left(x_{A, 2}^{*}\right)+\Delta=K_{2}^{A}\left(\tilde{x}_{1}\right)-\left(K_{1}^{A}\left(\tilde{x}_{1}\right)-K_{1}^{A}\left(x_{A, 1}^{*}\right)\right),
$$

what can be rewritten by virtue of (H.12) and (H.17) as follows:

$$
K_{2}^{A}\left(\bar{x}_{2}\right)-\bar{z}_{2}+\Delta=K_{2}^{A}\left(\tilde{x}_{1}\right)-\tilde{z}_{1}+\delta .
$$

Since $\Delta \geq \delta$ holds by assumption, it follows that the set of offers in question satisfies constraint (5). This finally proves the alternative set of offers to be feasible. Furthermore, it delivers a lower value to the objective function (1) than the original set of offers $\left(\bar{x}_{1}, \bar{z}_{1}, \bar{x}_{2}, \bar{z}_{2}\right)$ does. Indeed, following Sucky (2006, Section 4.2), it is straightforward to verify that the order size $\tilde{x}_{1}$ and the side payment $\tilde{z}_{1}$ defined in (H.16)-(H.17) uniquely minimize

$$
K^{P}\left(x_{1}\right)+z_{1} \quad \text { s.t. } \quad K_{1}^{A}\left(x_{1}\right)-z_{1} \leq K_{1}^{A}\left(x_{A, 1}^{*}\right)-\delta .
$$

Since the pair $\bar{x}_{1}, \bar{z}_{1}$ obviously satisfies the constraint in (H.18), we consequently have that

$$
K^{P}\left(\tilde{x}_{1}\right)+\tilde{z}_{1}<K^{P}\left(\bar{x}_{1}\right)+\bar{z}_{1} .
$$

But this obviously contradicts the optimality of the original set of offers $\left(\bar{x}_{1}, \bar{y}_{1}, \bar{x}_{2}, \bar{y}_{2}\right)$, and hence $\bar{x}_{1}=\mathrm{JELS}_{1}$ must indeed hold true. It can further be shown that the original set of offers coincides with the set of offers of type 3 if the latter is defined, or otherwise with the set of offers $7 \mathrm{a}$.

2) Assume that $\delta>\Delta$. We will prove that $\bar{x}_{2}=\mathrm{JELS}_{2}$. Assume the opposite: that $\bar{x}_{2} \neq \mathrm{JELS}_{2}$, and consider an alternative set of offers $\left(\bar{x}_{1}, \bar{z}_{1}, \tilde{x}_{2}, \tilde{z}_{2}\right)$ where

$$
\begin{aligned}
& \tilde{x}_{2}=\mathrm{JELS}_{2}, \\
& \tilde{z}_{2}=K_{2}^{A}\left(\tilde{x}_{2}\right)-K_{2}^{A}\left(x_{A, 2}^{*}\right) .
\end{aligned}
$$

This set of offers satisfies constraints (2), (3) due to equations (H.11), (H.20) respectively. 
Further, equations (H.12), (H.20) imply:

$$
K_{2}^{A}\left(\bar{x}_{2}\right)-\bar{z}_{2}=K_{2}^{A}\left(\tilde{x}_{2}\right)-\tilde{z}_{2},
$$

and therefore constraint (5) holds for the set of offers in question by virtue of (H.14). Furthermore, from (H.9) and (H.10) we obviously have:

$$
\bar{K}\left(\mathrm{JELS}_{2}\right)+\Delta \geq K_{1}^{A}\left(x_{A, 1}^{*}\right)-K_{2}^{A}\left(x_{A, 2}^{*}\right) .
$$

The above inequality can be rewritten by virtue of (H.2) and (H.19) as follows:

$$
K_{1}^{A}\left(x_{A, 1}^{*}\right) \leq K_{1}^{A}\left(\tilde{x}_{2}\right)-\left(K_{2}^{A}\left(\tilde{x}_{2}\right)-K_{2}^{A}\left(x_{A, 2}^{*}\right)\right)+\Delta .
$$

By virtue of (H.15) and (H.20), the above can be rewritten in the following form:

$$
K_{1}^{A}\left(\bar{x}_{1}\right)-\bar{z}_{1}+\delta \leq K_{1}^{A}\left(\tilde{x}_{2}\right)-\tilde{z}_{2}+\Delta .
$$

Since $\delta>\Delta$ holds by assumption, it follows that the set of offers in question satisfies constraint (4). This finally proves the alternative set of offers to be feasible. Furthermore, it delivers a lower value to the objective function (1) than the original set of offers $\left(\bar{x}_{1}, \bar{z}_{1}, \bar{x}_{2}, \bar{z}_{2}\right)$ does because the order size $\tilde{x}_{2}$ and the side payment $\tilde{z}_{2}$ defined in (H.19)-(H.20) uniquely minimize

$$
K^{P}\left(x_{2}\right)+z_{2} \quad \text { s.t. } \quad K_{2}^{A}\left(x_{2}\right)-z_{2} \leq K_{2}^{A}\left(x_{A, 2}^{*}\right),
$$

what is straightforward to verify following Sucky (2006, Section 4.2). But this contradicts the optimality of the original set of offers $\left(\bar{x}_{1}, \bar{y}_{1}, \bar{x}_{2}, \bar{y}_{2}\right)$, and hence $\bar{x}_{2}=\mathrm{JELS}_{2}$ must indeed hold true. It can further be shown that the original set of offers coincides with the set of offers 7 a.

Thus in both sub-cases above, we obtain that $\bar{x}_{i}=\operatorname{JELS}_{i}$ holds for at least one $i \in\{1,2\}$.

If the $1^{\text {st }}$ inequality in (H.1) does not hold, while the $2^{\text {nd }}$ does, we shall follow the same approach as in the above Case 1 , which implies $\bar{x}_{2}=\mathrm{JELS}_{2}$. The given set of offers $\left(\bar{x}_{1}, \bar{y}_{1}, \bar{x}_{2}, \bar{y}_{2}\right)$ can consequently be shown to coincide with set of offers 7 a.

Case 3. With $s=8 \mathrm{~b}$ we shall proceed similarly to the above Case 2. The given set of offers $\left(\bar{x}_{1}, \bar{y}_{1}, \bar{x}_{2}, \bar{y}_{2}\right)$ can further be shown to coincide with the set of offers of type 2 or the set of offers 8 a. This completes the proof. 


\section{Appendix I. Proof of Proposition 3}

The following lemma is helpful in proving the proposition.

Lemma I.1. Assume that the set of offers of type 1 satisfies the respective feasibility conditions as per Table 1. Then, this set of offers represents a unique optimal solution of problem (1)-(6).

Proof. Under the assumption of the lemma, the set of offers of type 1 represents a KKT solution of problem (1)-(6), and thus its feasible solution. Furthermore, this set of offers uniquely minimizes objective function (1) subject to constraints (2)-(3) (see Sucky, 2006, Section 4.2 for a proof). Hence it represents the unique optimal solution to (1)-(6) as well.

Let the hypothesis $\mathrm{JELS}_{1}=\mathrm{JELS}_{2}$ of the proposition hold. Assume first that $h_{A, 1}=h_{A, 2}$. Then, by definition of the joint economic lot sizes $\mathrm{JELS}_{1,2}$ in $(7)$, the equality $\mathrm{JELS}_{1}=\mathrm{JELS}_{2}$ implies $B_{1}=B_{2}$. This, however, contradicts the assumption $\theta_{1} \neq \theta_{2}$.

Thus, $h_{A, 1} \neq h_{A, 2}$. Then, by definition of the joint economic lot sizes JELS $\mathrm{J}_{1,2}$ in (7), we have $B_{1} \neq B_{2}$. Furthermore, it can be easily verified that either $B_{1}>B_{2}, h_{A, 1}>h_{A, 2}$ or $B_{1}<B_{2}, h_{A, 1}<h_{A, 2}$ holds - so that the buyer's possible cost structures strictly dominate one another in Pareto sense. Thus the order sizes

$$
x_{1}=x_{2}=\sqrt{2 d \cdot \frac{B_{1}-B_{2}}{h_{A, 1}-h_{A, 2}}}
$$

in the sets of offers 7a and 8a are defined and positive. We shall prove that the following holds:

$$
x_{1}=x_{2}=\mathrm{JELS}_{1}=\mathrm{JELS}_{2}
$$

Indeed, this is obviously equivalent to proving that

$$
\frac{S_{1}-S_{2}}{H_{1}-H_{2}}=\frac{S_{1}}{H_{1}}
$$

where $S_{1,2}, H_{1,2}$ are as defined in (13). We can transform the above equality to: $S_{1} H_{1}-S_{2} H_{1}=$ $S_{1} H_{1}-S_{1} H_{2}$, and subsequently to

$$
\frac{S_{1}}{H_{1}}=\frac{S_{2}}{H_{2}}
$$

what obviously holds true due to the hypothesis $\mathrm{JELS}_{1}=\mathrm{JELS}_{2}$ of the proposition. This proves (I.2) to hold. Consequently, both sets of offers 7a and 8a satisfy the respective necessary optimality conditions as per Table 1. It is also easy to see that in each of the menus 7a, 8a, the side payments obey $z_{1}=z_{2}$. Thus, both menus entail bunching. We below distinguish between Cases 1 to 3 . 
Case 1. Assume that the following holds:

$$
K_{1}^{A}\left(x_{1}\right)-K_{1}^{A}\left(x_{A, 1}^{*}\right)=K_{2}^{A}\left(x_{2}\right)-K_{2}^{A}\left(x_{A, 2}^{*}\right)
$$

where $x_{1}$ and $x_{2}$ satisfy (I.2). It is then straightforward to verify that the menus $7 \mathrm{a}$ and $8 \mathrm{a}$ coincide with each other and also with the set of offers of type 1 - that proves to satisfy the respective feasibility conditions. By virtue of Lemma I.1, this set of offers then represents the unique optimal solution of the bargaining problem (1)-(6). Next, we rewrite (I.5) in the following form:

$$
K_{1}^{A}\left(x_{1}\right)-K_{2}^{A}\left(x_{2}\right)=K_{1}^{A}\left(x_{A, 1}^{*}\right)-K_{2}^{A}\left(x_{A, 2}^{*}\right) .
$$

From the preceding analysis we know that the buyer's possible cost structures strictly dominate one another in Pareto sense. Assuming that $B_{1}>B_{2}, h_{A, 1}>h_{A, 2}$, and substituting $x_{A, i}^{*}=\sqrt{2 d B_{i} / h_{A, i}}$ and (I.1) into (I.6), it is straightforward to obtain:

$$
\sqrt{2 d\left(B_{1}-B_{2}\right)\left(h_{A, 1}-h_{A, 2}\right)}=\sqrt{2 d B_{1} h_{A, 1}}-\sqrt{2 d B_{2} h_{A, 2}}
$$

while the assumption $B_{1}<B_{2}, h_{A, 1}<h_{A, 2}$ leads in a similar way to:

$$
-\sqrt{2 d\left(B_{1}-B_{2}\right)\left(h_{A, 1}-h_{A, 2}\right)}=\sqrt{2 d B_{1} h_{A, 1}}-\sqrt{2 d B_{2} h_{A, 2}}
$$

In either case, squaring both sides of the equation, expanding the parentheses and simplifying gives:

$$
-B_{1} h_{A, 2}-B_{2} h_{A, 1}=-2 \sqrt{B_{1} h_{A, 1} B_{2} h_{A, 2}}
$$

what leads in an obvious way to the equality $\left(\sqrt{B_{1} h_{A, 2}}-\sqrt{B_{2} h_{A, 1}}\right)^{2}=0$ and, thus, to the equality $B_{1} h_{A, 2}=B_{2} h_{A, 1}$. From this and from (I.4) it is then easy to derive:

$$
\frac{R}{H_{P}}=\frac{B_{1}}{h_{A, 1}}=\frac{B_{2}}{h_{A, 2}}
$$

From this, it is easy to see that Case 1 is equivalent to:

$$
\mathrm{ELS}=\mathrm{EOQ}_{1}=\mathrm{EOQ}_{2}=\mathrm{JELS}_{1}=\mathrm{JELS}_{2}
$$

where ELS $\equiv x_{P}^{*}$ is the supplier's economic lot size and $\mathrm{EOQ}_{i} \equiv x_{A, i}^{*}$ is the economic order quantity corresponding to the buyer's cost structure no. $i, i=1,2$. Thus, the first-best outcome is realised in Case 1 without bargaining. This proves assertion i) of the proposition. 
Case 2. Assume now that the following holds:

$$
K_{1}^{A}\left(x_{1}\right)-K_{1}^{A}\left(x_{A, 1}^{*}\right)<K_{2}^{A}\left(x_{2}\right)-K_{2}^{A}\left(x_{A, 2}^{*}\right)
$$

where $x_{1}$ and $x_{2}$ satisfy (I.2), as before. It is then straightforward to verify that menu 7 a satisfies the respective feasibility condition and thus proves to be a KKT solution of the bargaining problem (1)-(6), while menu 8a does not. We will below conduct the proof of assertion ii) of the proposition by comparing the objective value that menu 7 a delivers to the bargaining problem with that of other KKT solutions, if there are any. Referring to Table 1 and taking into account assumption (I.11), it is straightforward to verify that:

- the set of offers of type 1 fails to satisfy the respective feasibility conditions;

- by virtue of (I.4), the set of offers of type 2 , if defined, coincides with menu 8 a and fails to satisfy the respective feasibility conditions;

- by virtue of (I.4), the set of offers of type 3 , if defined, coincides with menu 7a;

- the sets of offers of type 5 fail to satisfy the respective feasibility condition;

- by virtue of (I.3) and (I.4), menu $7 \mathrm{~b}$ coincides with menu 7a;

- by virtue of (I.3), menu $8 \mathrm{~b}$ coincides with menu $8 \mathrm{a}$ and therefore fails to satisfy the respective feasibility conditions.

Furthermore, we below prove that under the hypothesis of the proposition and assumption (I.11),

- the sets of offers of type 6 fail to satisfy the respective necessary optimality conditions.

We separate the proof of the latter assertion in the following two steps. Firstly, from the preceding analysis we know that the buyer's possible cost structures strictly dominate one another in Pareto sense. We shall prove that under assumption (I.11), specifically the following must hold:

$$
B_{1}>B_{2}, h_{A, 1}>h_{A, 2} \text {. }
$$

Assume the opposite: that $B_{1}<B_{2}, h_{A, 1}<h_{A, 2}$ holds, and rewrite (I.11) in the following form:

$$
K_{1}^{A}\left(x_{1}\right)-K_{2}^{A}\left(x_{2}\right)<K_{1}^{A}\left(x_{A, 1}^{*}\right)-K_{2}^{A}\left(x_{A, 2}^{*}\right) .
$$

By taking the same steps as in (I.6)-(I.8), it is straightforward to rewrite (I.13) as follows:

$$
-\sqrt{2 d\left(B_{1}-B_{2}\right)\left(h_{A, 1}-h_{A, 2}\right)}<\sqrt{2 d B_{1} h_{A, 1}}-\sqrt{2 d B_{2} h_{A, 2}},
$$


or, equivalently,

$$
\sqrt{\left(B_{1}-B_{2}\right)\left(h_{A, 1}-h_{A, 2}\right)}>\sqrt{B_{2} h_{A, 2}}-\sqrt{B_{1} h_{A, 1}}
$$

Since $B_{1}<B_{2}, h_{A, 1}<h_{A, 2}$ holds by assumption, both sides of the above inequality are positive. Squaring both sides and expanding the parentheses gives, after an obvious simplification:

$$
-B_{1} h_{A, 2}-B_{2} h_{A, 1}>-2 \sqrt{B_{1} h_{A, 1} B_{2} h_{A, 2}}
$$

what leads in an obvious way to the inequality

$$
\left(\sqrt{B_{1} h_{A, 2}}-\sqrt{B_{2} h_{A, 1}}\right)^{2}<0
$$

which is infeasible - a contradiction. Hence (I.12) must indeed hold true.

Next, consider a set of offers of type 6 as defined in Table 1 , and denote its order sizes by $\tilde{x}_{1}, \tilde{x}_{2}$. Referring to its necessary optimality conditions, it is straightforward to verify that condition

$$
x_{A, 1}^{*}=x_{A, 2}^{*}, \quad \tilde{x}_{1}=\mathrm{JELS}_{1}, \quad \tilde{x}_{2}=\mathrm{JELS}_{2}
$$

cannot be satisfied under the hypothesis $\mathrm{JELS}_{1}=\mathrm{JELS}_{2}$ of the proposition and assumption (I.11), while the alternative condition requires in particular that both of the following inequalities hold:

$$
\mathcal{M}_{1}\left(\tilde{x}_{2}\right):=\frac{H_{2} \tilde{x}_{2}^{2}-2 d S_{2}}{\bar{h}_{A} \tilde{x}_{2}^{2}-2 d \bar{B}} \geq 0 \quad \mathcal{M}_{2}\left(\tilde{x}_{1}\right):=-\frac{H_{1} \tilde{x}_{1}^{2}-2 d S_{1}}{\bar{h}_{A} \tilde{x}_{1}^{2}-2 d \bar{B}} \geq 0
$$

where

$$
\bar{B}=B_{1}-B_{2}>0 \quad \text { and } \quad \bar{h}_{A}=h_{A, 1}-h_{A, 2}>0
$$

By virtue of (13), (I.3), (I.4) we, however, have

$$
\frac{\bar{B}}{\bar{h}_{A}}=\frac{S_{1}}{H_{1}}=\frac{S_{2}}{H_{2}}
$$

so that

$$
\mathcal{M}_{1}\left(\tilde{x}_{2}\right)=\frac{H_{2} \tilde{x}_{2}^{2}-2 d S_{2}}{\bar{h}_{A} \tilde{x}_{2}^{2}-2 d \bar{B}}=\frac{\tilde{x}_{2}^{2}-2 d \frac{S_{2}}{H_{2}}}{\frac{\bar{h}_{A}}{H_{2}} \cdot\left(\tilde{x}_{2}^{2}-2 d \frac{\bar{B}}{\bar{h}_{A}}\right)} \equiv \frac{H_{2}}{\bar{h}_{A}}>0
$$


whereas

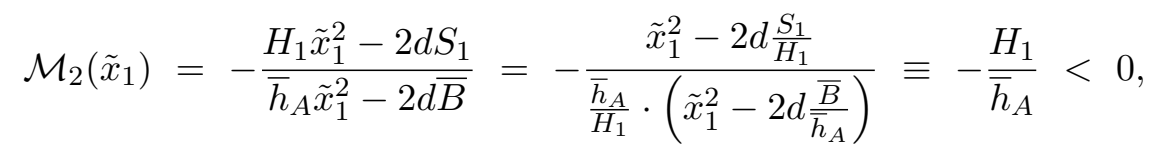

what proves that a set of offers of type 6 fails to satisfy the respective necessary optimality conditions, as required.

To summarize, the sets of offers of types 1, 2, 5, 6, 8 do not represent any KKT solutions, while the sets of offers of type 3 and 7b, if defined, coincide with menu 7a. Thus, it only remains to check the latter against the sets of offers of type 4 , as follows below.

By definition of a set of offers of type 4 in Table 1 , its order size $x_{1}=\mathrm{JELS}_{1}$ coincides with that of menu $7 \mathrm{a}$, while its second order size, denoted subsequently by $\tilde{x}_{2}$, is defined by one of the two possible expressions as follows:

$$
\tilde{x}_{2_{1,2}}=\sqrt{2 d} \cdot \frac{\sqrt{B_{1}} \pm \sqrt{B_{2}}}{\sqrt{h_{A, 1}} \pm \sqrt{h_{A, 2}}} .
$$

Due to assumption (I.11), a set of offers of type 4 automatically satisfies its feasibility condition. Referring to the necessary optimality conditions, it is straightforward to verify that condition

$$
x_{A, 1}^{*}=x_{A, 2}^{*} \quad \text { and } \quad \tilde{x}_{2}=\mathrm{JELS}_{2}
$$

cannot be satisfied given $\mathrm{JELS}_{1}=\mathrm{JELS}_{2}$ and assumption (I.11), while the alternative condition

$$
0 \leq \mathcal{M}_{1}\left(\tilde{x}_{2}\right) \leq \frac{\omega_{1}}{\omega_{2}}
$$

may or may not hold, depending on the problem data. Specifically, by virtue of (I.16), condition (I.19) holds whenever

$$
\frac{H_{2}}{\bar{h}_{A}} \leq \frac{\omega_{1}}{\omega_{2}}
$$

and does not hold otherwise. In the latter case, the sets of offers of type 4 fail to satisfy the necessary optimality conditions and, therefore, do not represent any KKT solutions. Then menu 7a happens to be the only KKT solution of the bargaining problem and its unique optimal solution.

Let now (I.20) hold. Then the set of offers of type 4 happens to be a KKT solution. Consider the objective value that it delivers:

$$
K_{4}^{P}:=\omega_{1} \cdot\left[K^{P}\left(x_{1}\right)+K_{1}^{A}\left(x_{1}\right)-K_{1}^{A}\left(x_{A, 1}^{*}\right)\right]+\omega_{2} \cdot\left[K^{P}\left(\tilde{x}_{2}\right)+K_{2}^{A}\left(\tilde{x}_{2}\right)-K_{2}^{A}\left(x_{A, 2}^{*}\right)\right] .
$$


We shall compare it with the objective value that menu 7a delivers:

$$
K_{7}^{P}:=\omega_{1} \cdot\left[K^{P}\left(x_{1}\right)+K_{2}^{A}\left(x_{1}\right)-K_{2}^{A}\left(x_{A, 2}^{*}\right)\right]+\omega_{2} \cdot\left[K^{P}\left(x_{2}\right)+K_{2}^{A}\left(x_{2}\right)-K_{2}^{A}\left(x_{A, 2}^{*}\right)\right],
$$

where $x_{2}=\mathrm{JELS}_{2}$, as before. The difference between the above two objective values is obviously

$$
\begin{aligned}
& K_{7}^{P}-K_{4}^{P}= \\
& =\omega_{1} \cdot\left[K_{1}^{A}\left(x_{A, 1}^{*}\right)-K_{2}^{A}\left(x_{A, 2}^{*}\right)-\left(K_{1}^{A}\left(x_{1}\right)-K_{2}^{A}\left(x_{1}\right)\right)\right]+\omega_{2} \cdot\left[K_{2}\left(x_{2}\right)-K_{2}\left(\tilde{x}_{2}\right)\right]= \\
& =\omega_{1} \cdot \underbrace{\left[K_{1}^{A}\left(x_{A, 1}^{*}\right)-K_{2}^{A}\left(x_{A, 2}^{*}\right)\right]}_{=\Delta_{1}}-\underbrace{\left[\omega_{1}\left(K_{1}^{A}\left(x_{1}\right)-K_{2}^{A}\left(x_{1}\right)\right)-\omega_{2} K_{2}\left(x_{2}\right)\right]}_{=\Delta_{2}}-\omega_{2} K_{2}\left(\tilde{x}_{2}\right),
\end{aligned}
$$

where $K_{2}(x)=K^{P}(x)+K_{2}^{A}(x)$. Denote the bracketed expressions in (I.21) by $\Delta_{1,2}$. By definition of cost functions involved and parameters $S_{2}, H_{2}, \bar{B}, \bar{h}_{A}$ in (13), (I.15), we can rewrite $\Delta_{2}$ as

$$
\Delta_{2}=\omega_{1}\left(\bar{B} \frac{d}{x_{1}}+\bar{h}_{A} \frac{x_{1}}{2}\right)-\omega_{2}\left(S_{2} \frac{d}{x_{2}}+H_{2} \frac{x_{2}}{2}\right)
$$

By substituting further the expression of $x_{1}$ as per (I.1) into the first parenthetical expression, and $x_{2}=\mathrm{JELS}_{2}=\sqrt{2 d S_{2} / H_{2}}$ into the second, it is straightforward to rewrite the above as

$$
\Delta_{2}=\omega_{1} \sqrt{2 d \bar{B} \bar{h}_{A}}-\omega_{2} \sqrt{2 d S_{2} H_{2}}=\sqrt{2 d \bar{B} \bar{h}_{A}} \cdot\left(\omega_{1}-\omega_{2} \sqrt{\frac{S_{2} H_{2}}{\bar{B} \bar{h}_{A}}}\right)
$$

By virtue of (I.3)-(I.4) and by definition of parameters $S_{i}, H_{i}, \bar{B}, \bar{h}_{A}$ we however have:

$$
\frac{S_{2}}{\bar{B}}=\frac{H_{2}}{\bar{h}_{A}},
$$

so that (I.22) can be rewritten as

$$
\Delta_{2}=\sqrt{2 d \bar{B} \bar{h}_{A}} \cdot\left(\omega_{1}-\omega_{2} \cdot \frac{H_{2}}{\bar{h}_{A}}\right) .
$$

Referring now to the last term in (I.21), let $\tilde{x}_{2}:=\tilde{x}_{2_{1}}$, as defined by (I.18); the other case can be treated in the same way. By definition of function $K_{2}(x)$ as well as by definition of parameters $S_{2}, H_{2}$ in (13), we then obtain:

$$
\begin{aligned}
& K_{2}\left(\tilde{x}_{2}\right)=S_{2} \frac{d}{\tilde{x}_{2}}+H_{2} \frac{\tilde{x}_{2}}{2}=S_{2} \sqrt{\frac{d}{2}} \cdot \frac{\sqrt{h_{A, 1}}+\sqrt{h_{A, 2}}}{\sqrt{B_{1}}+\sqrt{B_{2}}}+H_{2} \sqrt{\frac{d}{2}} \cdot \frac{\sqrt{B_{1}}+\sqrt{B_{2}}}{\sqrt{h_{A, 1}}+\sqrt{h_{A, 2}}}= \\
& =\sqrt{\frac{d}{2}} \cdot\left[S_{2} \cdot \frac{\left(\sqrt{h_{A, 1}}+\sqrt{h_{A, 2}}\right)\left(\sqrt{B_{1}}-\sqrt{B_{2}}\right)}{B_{1}-B_{2}}+H_{2} \cdot \frac{\left(\sqrt{B_{1}}+\sqrt{B_{2}}\right)\left(\sqrt{h_{A, 1}}-\sqrt{h_{A, 2}}\right)}{h_{A, 1}-h_{A, 2}}\right] .
\end{aligned}
$$


Taking (I.15) and (I.23) into account, we can rewrite the above in the following form:

$$
\begin{aligned}
& K_{2}\left(\tilde{x}_{2}\right)= \\
& =\sqrt{\frac{d}{2}} \cdot \frac{H_{2}}{\bar{h}_{A}} \cdot\left[\left(\sqrt{h_{A, 1}}+\sqrt{h_{A, 2}}\right)\left(\sqrt{B_{1}}-\sqrt{B_{2}}\right)+\left(\sqrt{B_{1}}+\sqrt{B_{2}}\right)\left(\sqrt{h_{A, 1}}-\sqrt{h_{A, 2}}\right)\right]= \\
& =\sqrt{2 d} \cdot \frac{H_{2}}{\bar{h}_{A}} \cdot\left[\sqrt{B_{1} h_{A, 1}}-\sqrt{B_{2} h_{A, 2}}\right] .
\end{aligned}
$$

From (I.6)-(I.7) we, however, know that

$$
\sqrt{2 d B_{1} h_{A, 1}}-\sqrt{2 d B_{2} h_{A, 2}}=K_{1}^{A}\left(x_{A, 1}^{*}\right)-K_{2}^{A}\left(x_{A, 2}^{*}\right),
$$

what allows us to rewrite (I.25) in the following form:

$$
K_{2}\left(\tilde{x}_{2}\right)=\frac{H_{2}}{\bar{h}_{A}} \cdot\left(K_{1}^{A}\left(x_{A, 1}^{*}\right)-K_{2}^{A}\left(x_{A, 2}^{*}\right)\right)=\frac{H_{2}}{\bar{h}_{A}} \cdot \Delta_{1} .
$$

By virtue of (I.24) and (I.26), we can then rewrite (I.21) as follows:

$$
\begin{aligned}
& K_{7}^{P}-K_{4}^{P}= \\
& =\left[K_{1}^{A}\left(x_{A, 1}^{*}\right)-K_{2}^{A}\left(x_{A, 2}^{*}\right)\right] \cdot\left(\omega_{1}-\omega_{2} \cdot \frac{H_{2}}{\bar{h}_{A}}\right)-\sqrt{2 d \bar{B} \bar{h}_{A}} \cdot\left(\omega_{1}-\omega_{2} \cdot \frac{H_{2}}{\bar{h}_{A}}\right)= \\
& =\left[K_{1}^{A}\left(x_{A, 1}^{*}\right)-K_{2}^{A}\left(x_{A, 2}^{*}\right)-\sqrt{2 d \bar{B} \bar{h}_{A}}\right] \cdot\left(\omega_{1}-\omega_{2} \cdot \frac{H_{2}}{\bar{h}_{A}}\right) .
\end{aligned}
$$

From (I.6)-(I.7) we, however, know that

$$
\sqrt{2 d \bar{B} \bar{h}_{A}}=\sqrt{2 d\left(B_{1}-B_{2}\right)\left(h_{A, 1}-h_{A, 2}\right)}=K_{1}^{A}\left(x_{1}\right)-K_{2}^{A}\left(x_{2}\right),
$$

what allows us finally to rewrite (I.27) as

$$
\begin{aligned}
& K_{7}^{P}-K_{4}^{P}= \\
& =\underbrace{\left[K_{1}^{A}\left(x_{A, 1}^{*}\right)-K_{2}^{A}\left(x_{A, 2}^{*}\right)-\left(K_{1}^{A}\left(x_{1}\right)-K_{2}^{A}\left(x_{2}\right)\right)\right]}_{>0} \cdot \underbrace{\left(\omega_{1}-\omega_{2} \cdot \frac{H_{2}}{\bar{h}_{A}}\right)}_{\geq 0} .
\end{aligned}
$$

Note that the bracketed expression in (I.28) is strictly positive due to assumption (I.11), while the parenthetical expression is non-negative by virtue of (I.20). Hence when (I.20) holds, the difference $K_{7}^{P}-K_{4}^{P}$ is nonnegative. Specifically, this difference is equal to zero if and only if (I.20) holds as equality. In this case a set of offers of type 4 delivers the same objective value as menu 7 a does, 
and thus both menus solve bargaining problem (1)-(6). By the convention adopted in Section 4, we assume that from several optimal sets of offers, the supplier picks one that minimizes the expected supply-chain costs. Since $x_{1}=x_{2}=$ JELS $_{1}=$ JELS $_{2}$ holds for menu 7 a due to (I.2), and since the joint economic lot size $\mathrm{JELS}_{i}$ is known to uniquely minimize the joint total cost function $K_{i}(x):=K^{P}(x)+K_{i}^{A}(x), i=1,2$, we conclude that menu 7a minimizes the expected supply-chain costs, while a menu of offers of type 4 does not - as it can be shown that assumption (I.11) implies $\tilde{x}_{2} \neq \mathrm{JELS}_{2}$. To summarize, Case 2 under consideration leads us to

Observation 3. Whenever condition (I.20) does not hold or holds as equality, menu 7a becomes offered by the supplier to the buyer, leading to bunching, while otherwise, due to a strictly positive difference $K_{7}^{P}-K_{4}^{P}$, a set of offers of type 4 becomes offered, which does not entail bunching.

Taking into account definitions of parameters $H_{1}, H_{2}, \bar{h}_{A}$ in (13) and (I.15), we finally note that condition (I.20) - referred to in Observation 3 - can be rewritten equivalently as follows:

$$
\begin{aligned}
& \frac{H_{2}}{\bar{h}_{A}} \leq \frac{\omega_{1}}{\omega_{2}} \leftrightarrow \frac{H_{1}-H_{2}}{H_{2}} \geq \frac{\omega_{2}}{\omega_{1}} \leftrightarrow \frac{H_{1}}{H_{2}} \geq 1+\frac{\omega_{2}}{\omega_{1}} \leftrightarrow \frac{H_{1}}{H_{2}} \geq \frac{1}{\omega_{1}} \leftrightarrow \\
& \leftrightarrow \quad \frac{H_{2}}{H_{1}} \leq \omega_{1} .
\end{aligned}
$$

Case 3. This case assumes that

$$
K_{1}^{A}\left(x_{1}\right)-K_{1}^{A}\left(x_{A, 1}^{*}\right)>K_{2}^{A}\left(x_{2}\right)-K_{2}^{A}\left(x_{A, 2}^{*}\right),
$$

where $x_{1}$ and $x_{2}$ satisfy (I.2), as before. It is then straightforward to verify that menu 8a satisfies the respective feasibility condition and thus proves to be a KKT solution, while menu 7a does not. This case can be analyzed in the same way as Case 2 above. In particular, it implies:

$$
B_{1}<B_{2}, h_{A, 1}<h_{A, 2},
$$

and leads to the following

Observation 4. Whenever condition

$$
\frac{H_{1}}{H_{2}} \leq \omega_{2}
$$

does not hold or holds as equality, menu 8 a becomes offered by the supplier to the buyer, leading to bunching, while otherwise a set of offers of type 5 becomes offered, which entails no bunching.

Thus, the analysis of Cases 2 and 3 implies assertion ii) of the proposition. 


\section{Appendix J. Proof of Proposition 4}

\section{J.1. Definitions and preliminary results}

Let $f(x)$ be here and below a real-valued function defined on a convex set $X \subseteq \mathbb{R}^{n}$. Then, $f$ is said to be quasiconvex if for each $x, y \in X$ and each $\lambda \in(0,1)$ the following holds:

$$
f(\lambda x+(1-\lambda) y) \leq \max \{f(x), f(y)\}
$$

It is known that a function is quasiconvex if and only if the level set $\{x \mid f(x) \leq \alpha\}$ is convex for every $\alpha \in \mathbb{R}$ (see e.g. Bazaraa et al., 2006; Mangasarian, 1969). Further, $f$ is said to be pseudoconvex if it is differentiable and for each $x, y \in X$ the following holds:

$$
\langle\nabla f(x), y-x\rangle \geq 0 \quad \text { implies } \quad f(y) \geq f(x),
$$

where $\langle\cdot, \cdot\rangle$ designates the inner product of two vectors. The relationship between convexity, pseudoconvexity and quasiconvexity is described by the following proposition (Mangasarian, 1965):

Proposition J.1. The following implications hold true for a differentiable function $f$ : convex $\Rightarrow$ pseudoconvex $\Rightarrow$ quasiconvex, but none of the converse is true.

A generalization to quasiconvexity (resp. pseudoconvexity) at a point $\bar{x}$ is obtained by holding $x=\bar{x}$ in the above definitions. The following proposition is a theorem by Mangasarian (1965), which we give here in a generalized form due to Bazaraa et al. (2006, p. 195).

Proposition J.2. Let $\bar{x}$ be a KKT solution of problem (P) defined in Appendix G, and let $I=$ $\left\{i \mid g_{i}(\bar{x})=0\right\}$. If $f$ is pseudoconvex at $\bar{x}$ and $g_{i}, i \in I$, are differentiable and quasiconvex at $\bar{x}$ then $\bar{x}$ is an optimal solution to $(\mathrm{P})$.

The following lemma is helpful in proving Proposition 4.

Lemma J.1. Let $\zeta(x)=K_{1}^{A}(x)-K_{2}^{A}(x)$. Assume that $\left(B_{1}, h_{A, 1}\right) \neq\left(B_{2}, h_{A, 2}\right)$. Then, with $B_{1} \leq B_{2}$ and $h_{A, 1} \geq h_{A, 2}$, the following holds:

a) $\zeta(x)$ is monotone increasing, concave and pseudoconvex.

b) $-\zeta(x)$ is monotone decreasing and convex.

With $B_{1} \geq B_{2}$ and $h_{A, 1} \leq h_{A, 2}$, the above assertions about $\zeta$ and $-\zeta$ must be interchanged.

Proof. Obviously, $\zeta(x)$ is differentiable on the domain of definition $\{x \mid x>0\}$. Let $B_{1} \leq B_{2}$ and $h_{A, 1} \geq h_{A, 2}$ hold. Then, obviously, $\zeta^{\prime}>0, \zeta^{\prime \prime} \leq 0$, and so $\zeta$ is monotone increasing and concave. It 
is pseudoconvex by its strict monotonicity, since for each $x, y>0$ the inequality

$$
\langle\nabla \zeta(x), y-x\rangle \equiv \zeta^{\prime}(x) \cdot(y-x) \geq 0
$$

can only be satisfied with $y \geq x$, what in turn implies $\zeta(y) \geq \zeta(x)$. This proves assertion a). The remaining assertions can be proven in a similar way.

\section{J.2. Proof of the proposition}

Assume that the hypothesis of the proposition holds. Consider a KKT solution $\left(\bar{x}_{1}, \bar{z}_{1}, \bar{x}_{2}, \bar{z}_{2}\right)$ of problem (1)-(6). By construction, it is represented by some set of offers of type 1 to 8 , as per Table 1, and satisfies the respective feasibility and necessary optimality conditions. To prove that it is optimal to (1)-(6), we below use the Generalized Lagrange Multiplier (GLM) approach (Everett, 1963) in its extended form due to Brooks and Geoffrion (1966). When applied to problem (P) in Appendix G, their result states that if there exist multipliers $\bar{\lambda}_{i} \geq 0, i=1, \ldots, m$, such that

i) $\bar{x} \in X$ delivers an unconstrained minimum to the restricted Lagrangian function

$$
\bar{L}(x)=f(x)+\sum \bar{\lambda}_{i} g_{i}(x)
$$

ii) $g_{i}(\bar{x}) \leq 0 \quad$ and $\quad \bar{\lambda}_{i} g_{i}(\bar{x})=0$ for all $i=1, \ldots, m$,

then $\bar{x}$ is optimal to (P) (see also Bazaraa et al., 2006, p. 212). Referring to problem (1)-(6), observe that, by definition of a KKT solution, there exists a feasible combination $\left(\bar{\lambda}_{1}, \bar{\lambda}_{2}, \bar{\mu}_{1}, \bar{\mu}_{2}\right)$ of Lagrange multipliers that satisfies KKT conditions (A.1)-(A.8) in conjunction with the given set of offers. Therefore, $\left(\bar{x}_{1}, \bar{z}_{1}, \bar{x}_{2}, \bar{z}_{2}\right)$ and $\left(\bar{\lambda}_{1}, \bar{\lambda}_{2}, \bar{\mu}_{1}, \bar{\mu}_{2}\right)$ together fulfill the above GLM condition ii) with respect to problem (1)-(6) (see also Bazaraa et al., 2006, pp. 209-210). We need to show that i) is fulfilled as well. For that, refer to the Lagrange function associated with problem (1)-(6):

$$
\begin{aligned}
& L\left(x_{1}, x_{2}, z_{1}, z_{2}, \lambda_{1}, \lambda_{2}, \mu_{1}, \mu_{2}\right)=\omega_{1} \cdot\left(K^{P}\left(x_{1}\right)+z_{1}\right)+\omega_{2} \cdot\left(K^{P}\left(x_{2}\right)+z_{2}\right) \\
& +\lambda_{1} \cdot\left(K_{1}^{A}\left(x_{1}\right)-z_{1}-K_{1}^{A}\left(x_{A, 1}^{*}\right)\right)+\lambda_{2} \cdot\left(K_{2}^{A}\left(x_{2}\right)-z_{2}-K_{2}^{A}\left(x_{A, 2}^{*}\right)\right) \\
& +\mu_{1} \cdot\left(K_{1}^{A}\left(x_{1}\right)-z_{1}-K_{1}^{A}\left(x_{2}\right)+z_{2}\right)+\mu_{2} \cdot\left(K_{2}^{A}\left(x_{2}\right)-z_{2}-K_{2}^{A}\left(x_{1}\right)+z_{1}\right) .
\end{aligned}
$$

By rearranging the terms in (J.1), rewrite it as follows:

$$
\begin{aligned}
& \omega_{1} \cdot K^{P}\left(x_{1}\right)+\omega_{2} \cdot K^{P}\left(x_{2}\right)+\left(\omega_{1}-\lambda_{1}-\mu_{1}+\mu_{2}\right) \cdot z_{1}+\left(\omega_{2}-\lambda_{2}+\mu_{1}-\mu_{2}\right) \cdot z_{2} \\
& +\left(\lambda_{1}+\mu_{1}\right) \cdot K_{1}^{A}\left(x_{1}\right)+\left(\lambda_{2}+\mu_{2}\right) \cdot K_{2}^{A}\left(x_{2}\right)-\lambda_{1} \cdot K_{1}^{A}\left(x_{A, 1}^{*}\right)-\lambda_{2} \cdot K_{2}^{A}\left(x_{A, 2}^{*}\right) \\
& -\mu_{1} \cdot K_{1}^{A}\left(x_{2}\right)-\mu_{2} \cdot K_{2}^{A}\left(x_{1}\right) .
\end{aligned}
$$


Note that KKT conditions (A.3)-(A.4) imply the following six equalities:

$$
\begin{array}{lllll}
\omega_{1}-\bar{\lambda}_{1}-\bar{\mu}_{1}+\bar{\mu}_{2}=0 & \Rightarrow & \bar{\lambda}_{1}+\bar{\mu}_{1}=\omega_{1}+\bar{\mu}_{2} & \Rightarrow & \bar{\lambda}_{1}=\omega_{1}-\bar{\mu}_{1}+\bar{\mu}_{2} \\
\omega_{2}-\bar{\lambda}_{2}+\bar{\mu}_{1}-\bar{\mu}_{2}=0 & \Rightarrow & \bar{\lambda}_{2}+\bar{\mu}_{2}=\omega_{2}+\bar{\mu}_{1} & \Rightarrow & \bar{\lambda}_{2}=\omega_{2}+\bar{\mu}_{1}-\bar{\mu}_{2}
\end{array}
$$

Substituting now $\bar{\lambda}_{1}, \bar{\lambda}_{2}, \bar{\mu}_{1}, \bar{\mu}_{2}$ into (J.2) and replacing $\bar{\lambda}_{1}, \bar{\lambda}_{2}$ with the respective expressions on the right in (J.3) yields the following expression of the restricted Lagrangian function $\bar{L}\left(x_{1}, z_{1}, x_{2}, z_{2}\right)$ :

$$
\begin{aligned}
& \omega_{1} \cdot\left(K^{P}\left(x_{1}\right)+K_{1}^{A}\left(x_{1}\right)-K_{1}^{A}\left(x_{A, 1}^{*}\right)\right)+\omega_{2} \cdot\left(K^{P}\left(x_{2}\right)+K_{2}^{A}\left(x_{2}\right)-K_{2}^{A}\left(x_{A, 2}^{*}\right)\right) \\
& -\bar{\mu}_{1} \cdot\left(K_{1}^{A}\left(x_{2}\right)-K_{2}^{A}\left(x_{2}\right)+K_{2}^{A}\left(x_{A, 2}^{*}\right)-K_{1}^{A}\left(x_{A, 1}^{*}\right)\right) \\
& -\bar{\mu}_{2} \cdot\left(K_{2}^{A}\left(x_{1}\right)-K_{1}^{A}\left(x_{1}\right)+K_{1}^{A}\left(x_{A, 1}^{*}\right)-K_{2}^{A}\left(x_{A, 2}^{*}\right)\right) .
\end{aligned}
$$

We will now treat different types of KKT solutions individually.

KKT solutions of type 1. By construction, it holds $\bar{\mu}_{1}, \bar{\mu}_{2}=0$ (Sucky, 2006, p. 531). Hence the last two terms in (J.4) vanish, and $\bar{L}$ turns out to be a convex function. Any stationary point of it hence represents its unconstrained minimum. By construction, the set of offers of type 1 is a stationary point of $\bar{L}$. Thus GLM condition i) is satisfied, and the set of offers is optimal to (1)-(6).

KKT solutions of type 4. By construction, $\bar{\mu}_{2}=0$, and the last term in (J.4) vanishes. We can assume that $\bar{\mu}_{1}>0$, since otherwise we deal with a KKT solution of type 1 . Note that $\bar{L}$ is convex unimodal in $x_{1}$, whereas for each fixed $x_{1}, \bar{L}$ is a sum of a convex and (by Lemma J.1 and Proposition J.1) a pseudoconvex function of $x_{2}$. We shall distinguish between two cases:

1. $B_{1} \leq B_{2}, h_{A, 1} \geq h_{A, 2}$. Then $\bar{L}$ is convex in $x_{2}$ due to convexity of functions $K^{P}, K_{2}^{A}$ and by virtue of Lemma J.1b. Furthermore, by definition of functions $K^{P}$ and $K_{i}^{A}, \bar{L}$ is unimodal in $x_{2}$ if and only if the following holds:

$$
\omega_{2} \cdot\left(\frac{d}{p} h_{P}+h_{A, 2}\right)>\bar{\mu}_{1} \cdot\left(h_{A, 1}-h_{A, 2}\right) .
$$

If $h_{A, 1}=h_{A, 2}$ then (J.5) is obviously satisfied. Otherwise, substitute $\bar{x}_{2}$ for $x_{2}$ in equation (B.6) of Appendix B, and substitute the right-hand side of (B.6) for $\bar{\mu}_{1}$ in (J.5). This gives:

$$
\omega_{2} \cdot\left(\frac{d}{p} h_{P}+h_{A, 2}\right)>\frac{\left(\frac{d}{p} h_{P}+h_{A, 2}\right) \bar{x}_{2}^{2}-2 d\left(R+B_{2}\right)}{\left(h_{A, 1}-h_{A, 2}\right) \bar{x}_{2}^{2}-2 d\left(B_{1}-B_{2}\right)} \cdot \omega_{2} \cdot\left(h_{A, 1}-h_{A, 2}\right) .
$$

Let $S_{2}, H_{2}$ and $\mathcal{A}$ be as defined in Table 1 as well as in eq. (13) and eq. (C.1) of Appendix C. 
Then (J.6) can be transformed to:

$$
H_{2}>\frac{H_{2} \bar{x}_{2}^{2}-2 d S_{2}}{\bar{x}_{2}^{2}-\mathcal{A}}
$$

Note that $\mathcal{A} \leq 0$. Hence the denominator in the right-hand side of (J.7) is positive, and therefore (J.7) can be transformed to:

$$
H_{2} \bar{x}_{2}^{2}-H_{2} \mathcal{A}>H_{2} \bar{x}_{2}^{2}-2 d S_{2},
$$

or, equivalently, to: $\mathcal{A}<2 d S_{2} / H_{2}$. This obviously holds true, and hence $\bar{L}$ is convex unimodal in $x_{2}$.

2. $B_{1} \geq B_{2}, h_{A, 1} \leq h_{A, 2}$. Then, by definition of functions $K^{P}$ and $K_{i}^{A}, \bar{L}$ is convex unimodal in $x_{2}$ if and only if the following holds:

$$
\omega_{2} \cdot\left(R+B_{2}\right)>\bar{\mu}_{1} \cdot\left(B_{1}-B_{2}\right)
$$

By taking a similar approach as in the above case 1, it can be shown that (J.8) holds true, and hence $\bar{L}$ is convex unimodal in $x_{2}$.

Thus, under the hypothesis of the proposition, $\bar{L}$ is separately convex unimodal in $x_{1}, x_{2}$ and constant in $z_{1}, z_{2}$. Given that $\bar{L}$ is separable, it obviously proves to be jointly convex in its variables, and hence its stationary points represent its unconstrained minima. By definition of a KKT solution, the set of offers under consideration is a stationary point of $\bar{L}$. Therefore, GLM condition i) is satisfied, and the set of offers in question is indeed optimal to (1)-(6).

KKT solutions of type 5 . By construction, $\bar{\mu}_{1}=0$. The proof follows in this case similar steps as those used above for KKT solutions of type 4 .

KKT solutions of type 6 . By construction, $\bar{\mu}_{1}, \bar{\mu}_{2} \geq 0$; we can, however, assume $\bar{\mu}_{1}, \bar{\mu}_{2}>0$ since otherwise the analysis reduces to one of the previously considered types of KKT solutions. We will show that under the hypothesis of the proposition, a set of offers of type 6 cannot satisfy the respective necessary optimality conditions. To proceed, recall that the values of Lagrange multipliers $\bar{\mu}_{1}, \bar{\mu}_{2}$ for a set of offers of type 6 are determined by eqs. (B.6), (B.9) in Appendix B, respectively.

Refer first to the case of $B_{1} \leq B_{2}, h_{A, 1} \geq h_{A, 2}$. Under this condition, the denominator in (B.6) is positive; hence the numerator must be positive as well, to ensure $\bar{\mu}_{1}>0$. This is obviously 
equivalent to requiring

$$
\bar{x}_{2}>\sqrt{2 d \cdot \frac{B_{2}+R}{h_{A, 2}+\frac{d}{p} h_{P}}},
$$

where the right-hand side represents the joint economic lot size $\mathrm{JELS}_{2}$. Refer now to (B.9). Under the same condition $B_{1} \leq B_{2}, h_{A, 1} \geq h_{A, 2}$, the denominator in (B.9) is negative; hence the numerator must be negative, as well, to ensure $\bar{\mu}_{2}>0$. This is obviously equivalent to requiring

$$
\bar{x}_{1}<\sqrt{2 d \cdot \frac{B_{1}+R}{h_{A, 1}+\frac{d}{p} h_{P}}},
$$

where the right-hand side represents the joint economic lot size JELS ${ }_{1}$. It is easy to verify that condition $B_{1} \leq B_{2}, h_{A, 1} \geq h_{A, 2}$ implies $\mathrm{JELS}_{1}<\mathrm{JELS}_{2}$, hence the strict inequality $\bar{x}_{1}<\bar{x}_{2}$ must necessarily hold. At the same time, $\bar{\mu}_{1}, \bar{\mu}_{2}>0$ implies that constraints (4)-(5) must be satisfied as equalities. Adding them together yields

$$
K_{1}^{A}\left(\bar{x}_{1}\right)-K_{2}^{A}\left(\bar{x}_{1}\right)=K_{1}^{A}\left(\bar{x}_{2}\right)-K_{2}^{A}\left(\bar{x}_{2}\right) .
$$

Thus we have an identical function in the both sides of the equation, evaluated at $\bar{x}_{1}$ and $\bar{x}_{2}$ respectively. However, this function is strictly monotone by Lemma J.1, and the equality can only hold with $\bar{x}_{1}=\bar{x}_{2}$. This contradicts the previously derived inequality $\bar{x}_{1}<\bar{x}_{2}$.

The case of $B_{1} \geq B_{2}, h_{A, 1} \leq h_{A, 2}$ can be analysed in the same way. Thus, it is not possible to have a set of offers of type 6 satisfying the respective necessary optimality condition under the hypothesis of the proposition, unless it coincides with one of the sets of offers considered above.

We now turn to the remaining four types no. 2, 3 and 7, 8 of KKT solutions.

KKT solutions of type 2. Note that KKT condition (A.4) implies:

$$
\bar{\mu}_{2}=\omega_{2}-\bar{\lambda}_{2}+\bar{\mu}_{1}
$$

Substituting this into (J.4) and simplifying yields:

$$
\begin{aligned}
& \omega_{1} \cdot\left(K^{P}\left(x_{1}\right)+K_{2}^{A}\left(x_{1}\right)\right)+\omega_{2} \cdot\left(K^{P}\left(x_{2}\right)+K_{2}^{A}\left(x_{2}\right)\right)+K_{1}^{A}\left(x_{1}\right)-K_{2}^{A}\left(x_{1}\right)-K_{1}^{A}\left(x_{A, 1}^{*}\right) \\
& -\bar{\lambda}_{2} \cdot\left(K_{1}^{A}\left(x_{1}\right)-K_{2}^{A}\left(x_{1}\right)+K_{2}^{A}\left(x_{A, 2}^{*}\right)-K_{1}^{A}\left(x_{A, 1}^{*}\right)\right) \\
& -\bar{\mu}_{1} \cdot\left(K_{2}^{A}\left(x_{1}\right)-K_{1}^{A}\left(x_{1}\right)+K_{1}^{A}\left(x_{2}\right)-K_{2}^{A}\left(x_{2}\right)\right) .
\end{aligned}
$$


At the same time, $\bar{\lambda}_{2}=\bar{\mu}_{1}=0$ holds for a KKT solution of type 2 by construction, and the last two terms in (J.9) vanish. Then, obviously, function $\bar{L}$ expressed by (J.9) is convex unimodal in $x_{2}$, whereas for each fixed $x_{2}, \bar{L}$ is a sum of a convex and (by Lemma J.1 and Proposition J.1) a pseudoconvex function of $x_{1}$. We can distinguish between four cases:

1. $B_{1} \leq B_{2}, h_{A, 1} \geq h_{A, 2}$. Then, by definition of functions $K^{P}$ and $K_{i}^{A}$, it is easy to verify the following:

a) if $\omega_{1} \cdot\left(R+B_{2}\right)>B_{2}-B_{1}$ then $\bar{L}$ is convex unimodal in $x_{1}$;

b) otherwise $\bar{L}$ is concave and strictly increasing in $x_{1}$.

2. $B_{1} \geq B_{2}, h_{A, 1} \leq h_{A, 2}$. Then $\bar{L}$ is convex in $x_{1}$ due to convexity of functions $K^{P}, K_{2}^{A}$ and by virtue of Lemma J.1. Furthermore, by definition of functions $K^{P}$ and $K_{i}^{A}$, it is easy to verify the following:

a) if $\omega_{1} \cdot\left(\frac{d}{p} h_{P}+h_{A, 2}\right)>h_{A, 2}-h_{A, 1}$ then $\bar{L}$ is unimodal in $x_{1}$;

b) otherwise $\bar{L}$ is strictly decreasing in $x_{1}$.

Thus under the hypothesis of the proposition, $\bar{L}$ is either convex unimodal (cases 1a, 2a) or strictly monotone in $x_{2}$ and cannot have a stationary point (cases $1 \mathrm{~b}, 2 \mathrm{~b}$ ). A conclusion can be drawn that cases $1 \mathrm{~b}$ and $2 \mathrm{~b}$ cannot apply - because the set of offers in question represents, by definition of a KKT solution, a stationary point of $\bar{L}$. In the remaining cases 1 a and 2 a we deal with a convex function $\bar{L}$ whose stationary points thus represent its unconstrained minima. Thus, GLM condition i) is satisfied, and the set of offers in question is optimal to (1)-(6).

KKT solutions of type 3. By construction, $\bar{\lambda}_{1}=\bar{\mu}_{2}=0$. The proof is conducted by substituting $\bar{\mu}_{1}=\omega_{1}-\bar{\lambda}_{1}+\bar{\mu}_{2}$ into (J.4) and following the steps of the above proof for a KKT solution of type 2 . KKT solutions of types 7 and 8 . By definition of KKT solutions of types 7 and $8, \bar{x}_{1}$ and $\bar{x}_{2}$ have to satisfy

$$
\bar{x}_{1} \bar{x}_{2}=\mathcal{A}
$$

where $\mathcal{A}$ is as defined in eq. (C.1), see also Table 1 . However, under the hypothesis of the proposition, $\mathcal{A}$ is either undefined (when $h_{A, 1}=h_{A, 2}$ ) or it holds that $\mathcal{A} \leq 0$, and therefore KKT solutions of types 7 and 8 are undefined. This completes the proof. 